Running Head: PERSON-ENVIRONMENT RELATIONS

\title{
Capturing Interactions, Correlations, Fits, and Transactions: \\ A Person-Environment Relations Model
}

\author{
John F. Rauthmann \\ Universität Bielefeld
}

To appear in the

Handbook of Personality Dynamics and Processes

(Edited by J. F. Rauthmann; published by Elsevier)

- Accepted version before final copy-editing -

Rauthmann, J. F. (in press). Capturing Interactions, Correlations, Fits, and Transactions: A Person-Environment Relations Model. In J. F. Rauthmann (Ed.), Handbook of Personality Dynamics and Processes. Elsevier.

Version date: May 20, 2020

49,817 words (172 pages)

5 boxes

9 tables

10 figures

Supplemental materials: Color figures can be found openly available at osf.io/ds6mw.

\section{Author Notes}

This chapter has been reviewed by Gabriela Blum, Christian Kandler, Niclas Kuper, and Manfred Schmitt. I thank them for their thorough as well as incredibly helpful and valuable feedback that has strengthened the chapter considerably. Any omissions and errors lie of course solely within the author's responsibility and fault. 


\begin{abstract}
To understand personality dynamics, processes, and functioning better, knowledge on the relations between persons and environments needs to advance. First, this chapter outlines basic elements of persons (short-term $\mathbb{P}$ as states and long-term $\mathbb{P}$ as traits) and environments (shortterm $\mathbb{E}$ for situations and long-term $\mathbb{e}$ for niches) along with their respective properties in a respecified and extended Lewinian formula. Second, a generic Person-Environment Relations Model (PERM) is presented that specifies the different relations and important effect paths between person, environment, and outcome variables. Within the PERM, four central types of person-environment relations are distinguished and subsequently discussed in detail: interactions (person and environment variables moderate each other's effects on outcomes), correlations (person and environment variables are concurrently associated), fits (person and environment variables match with each other), and transactions (person and environment variables affect each other across time). Here, person-environment fit is distinguished as either a special effect pattern (involving an interaction) or a correlation, respectively. Third, focusing on the different ways in which persons and environments calibrate themselves towards each other, different navigation mechanisms are systematized, with a special emphasis on how persons navigate environments. Lastly, suggestions and recommendations for future lines of theory, methodology, and empirical research are provided.
\end{abstract}

Keywords: persons, personality, traits, states, environments, situations, niches, personenvironment interactions, person-environment correlations, person-environment fit, personenvironment transactions 


\section{Table of Contents}

1. Introduction

2. Re-specifying the Lewinian Formula

2.1 Ingredients

2.1.1 Basic Elements: Person and Environment Variables

2.1.2 Differentiations: Flections of Measurement

2.1.3 Combinations

2.2 Functional Configurations

3. A General Person-Environment Relations Model (PERM)

4. Person-Environment Relations

4.1 Person-Environment Interactions

4.1.1 Analysis of Variance

4.1.2 Linear Interaction Effects

4.1.3 Non-linear Effects

4.1.4 Effect Patterns

4.1.5 Outcome-based, Variable-oriented Person-Environment Fit

4.1.6 Summary and Findings

4.2 Person-Environment Correlations

4.2.1 Variable-oriented Correlations

4.2.1.1 Behavior Genetics

4.2.1.2 Work

4.2.1.3 Relationships

4.2.1.4 Social Investments

4.2.1.5 Daily Life

4.2.1.6 Simulations

4.2.2 Person-oriented Profile Correlations

4.3 Person-Environment Fit

4.3.1 Important Distinctions

4.3.2 Processes and Consequences of Person-Environment Fit

4.4 Person-Environment Transactions

4.4.1 Timescales of Variables

4.4.1.1 Ontogenetic Processes

4.4.1.2 Manifestive/Expressive Processes

4.4.1.3 Actualgenetic Processes 
4.4.1.4 Accumulative/Accretive Processes

4.4.1.5 Summary

4.4.2 Transaction Effect Patterns across Time

4.4.2.1 Findings

4.4.2.1.1 Life Events

4.4.2.1.2 Work

4.4.2.1.3 Relationships

4.4.2.1.4 Daily Life

4.4.2.2 Summary

5. Person-Environment Calibrations and Navigation Mechanisms

5.1 Calibration Types

5.2 Navigation Mechanisms

5.2.1 Navigation Mechanisms for Person $\rightarrow$ Environment Calibrations

5.2.1.1 A Systematic Account

5.2.1.2 Empirical Evidence

5.2.2 Navigation Mechanisms for Environment $\rightarrow$ Person Calibrations

6. Recommendations and Future Directions

6.1 Taking Environment Assessment Seriously

6.2 Employing Appropriate Research Designs

6.3 Zooming into Processes, Sequences, and Cycles

6.4 Explanation vs. Prediction and Causality

6.5 Conceptualizing, Measuring, and Studying Calibrations

and Navigation Mechanisms

6.6 Distinguishing Different Types of Person-Environment Fit

6.7 A Transactional Perspective on Traits and Personality

6.8 Formalization

6.9 Theory, Multidisciplinary Integration, Replication, and Collaboration

6.10 Working Towards Principles of Person-Environment Relations

7. Conclusion

References

Boxes (1-5)

Tables (1-9)

Figures (1-10) 


\section{Introduction}

As psychologists, we want to describe, explain, predict, and sometimes even change the psychological structures, processes, dynamics, and functioning of persons. To do so, we need to understand how persons and environments are related to each other. Indeed, different literatures acknowledge the intricate, complex, and dynamic interplay between persons and their social or non-social environments (e.g., Allport, 1961; Altman \& Rogoff, 1987; Asendorpf \& Rauthmann, 2020; Back et al., 2011; Balliet, Tybur, \& van Lange, 2017; Baltes, 1997; Bandura, 1978, 1982; Baumert et al., 2017; Beck \& Jackson, 2020; Benet-Martínez, Chapter 10; Blum \& Schmitt, 2020; Bowers, 1973; Briley et al., 2019; Buss, 1987; Caspi, 1988; Caspi \& Bem, 1990; Caspi, Bem, \& Elder, 1989; Caspi \& Roberts, 1999, 2001; Craik, 1994, 2000; Cramer et al., 2012; Denissen \& Penke, 2008; DeYoung, 2015; Fleeson, 2012; Fleeson \& Jayawickreme, 2015; Fournier, Moskowitz, \& Zuroff, 2008, 2009; Fraley \& Roberts, 2005; Funder, 2001, 2006, 2008, 2009; Hankin \& Abramson, 2001; Hogan \& Roberts, 2000; Holleinstein \& Lougheed, 2013; Ickes, Snyder, \& Garcia, 1997; Jayawickreme, Zachry, \& Fleeson, 2019; Laceulle \& van Aken, 2018; Laland, 2004; Law et al., 1996; Lerner, 1978; Little, 1987; Magnusson, 1990; Magnusson \& Endler, 1977a,b; Markus \& Kitayama, 2010; Meagher, 2020; Mischel, 1977; Mischel \& Shoda, 1995, 1998, 2008; Molho \& Balliet, 2020; Pettigrew, 1997; Pincus, Hopwood, \& Wright 2020; Plomin et al., 1977; Rauthmann, 2015a; Rauthmann \& Sherman, 2016, 2020; Roberts, 2018; Roberts, \& Pomerantz, 2004; Roberts \& Nickel, in press; Ross \& Nisbett, 2011; Runyan, 1978; Ryff, 1987; Saegert \& Winkel, 1990; Sameroff, 1975; Scarr, 1996; Stokols \& Shumaker, 1981; Tett et al., 2013; Turkheimer, 2000; Walsh, Craik, \& Price, 2000; Wrzus, Chapter 5; Wrzus \& Roberts, 2017; Zaccaro et al., 2018). Further, many chapters in this handbook reference or even declare person-environment relations as central to personality psychology. From that vantage, personality dynamics and processes (e.g., Blum, Baumert, \& Schmitt, Chapter 2; Revelle, 1995, Chapter 1; Hampson, 2012; Hopwood, 2018) can only be properly understood if we advance our knowledge on person-environment relations. 
Consider Anna whose level of outgoing behavior (e.g., making eye contact with other

27 persons, talking to them, sending open non-verbal cues) has been measured across 34 occasions (Figure 1). Additionally, the kind of situation Anna was in was also measured at each occasion. This allows segmenting the measured snippets of Anna's life stream into different situational episodes. As can be seen, five episodes were sampled for Anna, and these episodes could be grouped into "Situation Class A" (blue dots; e.g., tasks need to be done), "Situation Class B" (green boxes; e.g., friend is talking to her), and "Situation Class C" (yellow triangles; e.g., friend helps her with tasks) according to their cues or psychological characteristics (Rauthmann, 2015b). To provide an account of Anna's consistency or variability (Beck \& Jackson, Chapter 4; Geukes et al., 2017), one could compare Anna's behavior within each episode (how does it fluctuate within an episode?) and across episodes (how does it fluctuate when similar episodes occur, and when different ones do?). This is an intra-individual perspective and an exercise in idiographic modeling. By also sampling corresponding information from several other participants, we could compare persons to each other or use nomothetic modeling from an interindividual perspective. Idiographic and nomothetic approaches are not mutually exclusive and can be integrated (Wright \& Zimmermann, 2019), but for simplicity the current example is just framed for one person, Anna. Apart from quantifying consistency and stability versus change in Anna's behaviors, other questions may also be interesting: Why is Anna in these situations in the first place? Why do her situations change? How do her behaviors and her situations relate to her mental functioning, her personality traits, and her relationships, country, and culture? How does Anna influence her situations, and how do they influence her? Does Anna craft herself a certain niche by the way she influences her situations, and does that niche have implications for Anna's development? How well does Anna fit into her environment, and does that fit have consequences for her well-being, identity, and social standing? 
- Figure $1-$

These and many more questions can be answered from the perspective of relating persons to environments (and vice versa) across time. Towards this end, this chapter first outlines basic elements and their properties based on a re-specified Lewinian formula. Next, a basic and generic Person-Environment Relations Model (PERM) is presented that systematizes the different relations and effect paths between person and environment variables. Within that model, central types of person-environment relations are distinguished: interactions, correlations, fits, and transactions. It will also be shown that person-environment fit can be understood in terms of interactions or correlations. Zooming in on the different ways in which persons and environments calibrate themselves and each other, different navigation mechanisms are outlined that help understand processes and dynamics involved in person-environment relations better. Lastly, several suggestions and recommendations for future lines of theory, methods, and empirical research are made. Throughout the chapter, the PERM is also made more concrete at various instances to showcase its flexibility and how it can be adapted to capture more nuanced phenomena. A summary of this chapter's contents can be found in Table 1 to help readers locate the topics and information contained in this chapter better.

- Table 1 -

\section{Re-specifying the Lewinian Formula}

It has become almost a truism to state that behavior results from the interplay of person and environment variables. Over eight decades ago, Lewin (1936) coined a now seminal formula capturing this idea:

$$
\mathrm{B}=f(\mathrm{P}, \mathrm{E})
$$

In this formula, behavior $(\mathrm{B})$ is a function of a person's current mental states $(\mathrm{P})$ and the current environment (E). In Lewin's (1936) words, “every psychological event depends upon the 
state of the person and at the same time on the environment, although their relative importance is different in different cases" (p. 12). The Lewinian formula in Equation 1 can also be condensed to $\mathrm{B}=f(\mathrm{~S})-$ where behavior is a function of the psychological or whole situation $(\mathrm{S})$ of a person. Further, because $\mathrm{P}$ and $\mathrm{E}$ are interdependent, it also holds that $\mathrm{E}=f(\mathrm{P})$ and $\mathrm{P}=f(\mathrm{E}) .^{1}$ Lewin's simple formula - describing an actualgenetic process (i.e., something that unfolds at a circumscribed and usually shorter moment in time) - has been misunderstood, reconceptualized, or extended several times (Bond, 2013; Funder, 2006, 2008, 2009; Wagerman \& Funder, 2009). For example, $\mathrm{P}$ has been interpreted as momentary behavioral states, behavioral patterns, personality, traits, or even genes and nature, and $\mathrm{E}$ as the psychological situation, perceived environment, context, socio-ecological niche, or even nurture. Undeniably, all of these “elements" are important in understanding human conditions, psychological functioning, daily dynamics, and developmental trajectories. However, they were not explicitly included in Lewin's simplified formula. ${ }^{2}$ Further, different issues are mixed, obscured, or even confused with each other, such as (1) overt behavior vs. covert mental operations, (2) structures vs. processes, (3) objective (actual) vs. subjective (perceived) accounts of the elements, (4) actualgenetic shortterm, momentary, fast-changing vs. ontogenetic long-term, enduring, slow-changing timescales of elements, (5) predictors (explanantia) vs. outcomes (explananda), and (6) systematic vs. unsystematic variance in the elements. A more inclusive and nuanced understanding of the basic elements and their relations will have to take these issues into account. The following revised formula and its concretizations in this chapter attempt to be a first step into such a direction:

$$
\mathbb{O}=f(\mathbb{P}, \mathbb{P}, \mathbb{E}, \mathbb{e})
$$

\footnotetext{
${ }^{1}$ These formulae simplistically assume that one variable can be fully explained by the other variable. Of course, such full explanation would be rare and residual components $R$ (e.g., unexplained systematic and unsystematic variance) would also need to be distinguished: $\mathrm{E}=f\left(\mathrm{P}, \mathrm{E}_{R}\right)$ and $\mathrm{P}=f\left(\mathrm{E}, \mathrm{P}_{R}\right)$, where the residual components $\mathrm{E}_{R}$ and $\mathrm{P}_{R}$ are independent of each other. Residual components are omitted from further equations.

${ }^{2}$ They may have been implied in Lewin's concept of a person's "life space" which are all inner and outer influences on a person.
} 
where $\mathbb{O}=$ set of outcomes variables, $\mathbb{P}=$ set of person state variables, $\mathbb{P}=$ set of person trait variables, $\mathbb{E}=$ set of momentary situation variables, $\mathbb{e}=$ set of enduring niche variables. ${ }^{3}$

\subsection{Ingredients}

\subsubsection{Basic Elements: Person and Environment Variables. Table 2 contains an} overview of the basic elements in Equation 2 and which forms they can each take. Generally, two broad types of variable groups can be distinguished (Asendorpf \& Rauthmann, 2020): person ( $\mathbb{P}$, P) and environment $(\mathbb{E}, \mathbb{e})$ variables. ${ }^{4}$

$\mathbb{P}$ denotes, in line with the original Lewinian formula, any momentary person conditions that can change rapidly or at least in short periods of time. Somewhat as a deviation from Lewin, however, $\mathbb{P}$ can be any kind of momentary person state (e.g., physiological, sensory, perceptual, cognitive, affective, motivational, volitional, intentional, regulatory, behavioral), not just "mental" ones. p denotes any regular, recurrent, or only slowly changing person variables. Such variables have been broadly related to morphology (e.g., body mass index, physical health, longevity), dispositions (e.g., Big Five, intelligence), characteristic adaptations (e.g., goals, values, attitudes, well-being), and narratives (e.g., self-concepts, identity) in the literature (McAdams \& Pals, 2006). P variables have elsewhere been referred to as states and P variables as traits (e.g., Cattell, Cattell, \& Rhymer, 1947; Fleeson, 2001; Roberts, 2018; Steyer, Ferring, \& Schmitt, 1992; Steyer, Schmitt, \& Eid, 1999), and this chapter follows this tradition. Importantly, traitness vs. stateness may reside on a continuum, and any person variable may lie on said

\footnotetext{
${ }^{3}$ The letters with double lines $(\mathbb{O}, \mathbb{P}, \mathbb{P}, \mathbb{E}, \mathbb{e}$ ) denote sets of variables regardless of their "flections" (see 2.1.2 Differentiations: Flections of Measurement). They are thus undifferentiated placeholders that could be any form of a specific variable. For example, $\mathbb{P}$ refers to any kind of momentary person variable (and could denote $\mathrm{P}, \overline{\mathrm{P}}, \dddot{\mathrm{P}}$, or $\dddot{\mathrm{P}}$; see Table 2), while a P refers to a specific momentary person variable measured once.

${ }^{4}$ Of note, Asendorpf and Rauthmann (2020) reversed what capitalized and decapitalized letters meant. There, and in contrast to this chapter, capitalized letters denoted more stable, long-term, or slowly changing variables, while decapitalized letters denoted less stable, short-term, or fast changing variables. Because Lewin's concepts have precedent, I decided to keep capitalized letters for the short-term variables he described and use decapitalized letters for the long-term ones instead. This also creates greater consistency with other literatures.
} 
113 continuum (Leising et al., 2014) and could potentially even be operationalized as both a trait and

114 a state (Fleeson, 2001). Indeed, any measured variable (e.g., even a body mass index) may

115 contain both state and trait components (Steyer et al., 1999), and it also depends on the properties

116 of measurement whether traits can be feasibly extracted (e.g., from repeated measurements).

$\mathbb{E}$ denotes, in line with the original Lewinian formula, any momentary environment

118 conditions that can change rapidly or at least in short periods of time (labeled here: situations).

119 These $\mathbb{E}$ variables can be measured in more objective terms (e.g., attending to the actual cues in

120 an environment) or in subjective/perceived terms (e.g., asking persons to rate psychological

121 characteristics of an environment). e denotes any regular, recurrent, or only slowly changing

122 environment conditions (labeled here: niches). Terms for $\mathbb{E}$ and $\mathbb{e}$ have not been used precisely or

123 consistently throughout the literature (Rauthmann, Sherman, \& Funder, 2015a). For example, $\mathbb{E}$

124 variables have elsewhere been referred to as situations, events, occurrences, or episodes, and $e$

125 variables as environments, niches, or contexts (Rauthmann, Sherman, \& Funder, 2015b). While $\mathbb{E}$

126 and e variables can be qualitatively different, it is often a matter of operationalization and

127 measurement whether one can distinguish more enduring e components from more variable $\mathbb{E}$

128 components in assessments of the environment - similar to how trait and state components are

129 inherent in every measurement of a person variable. Environment variables can be quite

130 heterogeneous. For example, they could represent (1) circumscribed ecological phenomena or

131 elements (e.g., nutrition, toxins, resource scarcity, natural hazards, catastrophes, weather

132 conditions, flora, fauna, architecture), (2) discrete life events (e.g., graduation, new job, marriage,

133 child birth, divorce, partner death, abuse, lottery win), (3) situational episodes (e.g., being at a

134 party), (4) social niches (e.g., one's online and offline social networks, friends, romantic partners,

135 work colleagues, groups), (5) institutions (e.g., vocations, occupations, organizations), (6) culture

136 (e.g., norms, traditions, rules, laws, roles, games), or even (7) history (e.g., epoch, zeitgeist). 
(10) can be any outcome variable of interest, which will usually reside either at the person

138 level $(\mathbb{P}, \mathbb{P})$ or at the environment level $(\mathbb{E}, \mathbb{e})$. Some research tends to structure outcomes into

139 individual (intrapersonal), interpersonal, and social-institutional domains (Ozer \& Benet-

140 Martínez, 2006; Soto, 2019). To a certain extent, designating which person or environment

141 variable serves as an outcome variable and which as a predictor may be arbitrary. Therefore,

142 theory and conceptual reasoning as well as appropriate methods (e.g., experimental and

143 longitudinal designs) should be used to establish clear predictor-outcome relations (see also

144 Hampson, Chapter 3; Mõttus, 2016; Wessels, Zimmermann, \& Leising, 2016).

To give an example of how person, environment, and outcome variables come together,

146 being approached by others at a social event (here, $\mathbb{O}$ is defined as an E) could be described or

147 explained as a function of currently being in a good mood $(\mathrm{P})$, being generally outgoing (p),

148 being in easy-going and pleasant social interactions (E), and already having a supporting social

149 network of friends (e). Because terms have been used haphazardly and inconsistently throughout

150 the literature (Asendorpf \& Rauthmann, 2020) and this chapter cannot engage in a full and

151 balanced treatise of terminologies, I will henceforth simply refer to the basic elements as person

152 variables $\mathbb{P}($ states $)$ and $\mathbb{P}($ traits) as well as $\mathbb{E}$ (situations and their cues or characteristics) and $e$

153 (niches and their cues or characteristics).

154

155

156

157
2.1.2 Differentiations: Flections of Measurement. As can be seen in Table 2, several measurement flections of $\mathbb{P}, \mathbb{P}, \mathbb{E}$, and $\mathbb{e}-$ which are used here as placeholders to be specified more clearly - can be differentiated based on measurement, distinguishing between (1) variableoriented (with no diacritics) vs. person-oriented (with ${ }^{\cdots}$ ) and (2) single-moment (with no diacritics) vs. repeated measurements (with ${ }^{-}$). In all instances, person variables may be assessed by different BIOPSIES data sources (actual Behavior and behavioral residues, Indirect measures, Observation, Physiological and biological measures, Strangers' impressions, 
161 Informants' knowledge, Experience sampling and ambulatory assessments, Self-ratings; see

162 Rauthmann, 2017), while environment variables can be measured, for example, by human raters

163 or sensors (Rauthmann, Sherman, \& Funder, 2020). However, these distinctions - often also

164 boiling down to more objective vs. consensual vs. subjective accounts of the variables - will not

165 be notified here to avoid even further complexity. However, they could be easily integrated by

166 using subscripts, such as, for example, $\mathrm{P}_{\text {self }}$ for a self-rating of a single person state at one point in

167 time (e.g., how extraverted a person believes she or he is at a given moment of measurement).

First, P, p, E, and e are "simple" variable-oriented measurements at one measurement occasion - one variable is sampled at a specific point in time. Second, $\overline{\mathrm{P}}$ and $\overline{\mathrm{E}}$ are derived from repeated variable-oriented measurements (such as in ambulatory assessment or experience sampling) of several $\mathrm{P}$ and E variables, respectively. Such data make it possible to construct different new scores from repeatedly measured variables. For example, density distributions of these variables can be formed which are described by certain parameters (e.g., arithmetic mean, median, mode, standard deviation, minimum, maximum, skewness, kurtosis; see Fleeson, 2001 and Fleeson \& Gallagher, 2009 for an application to Ps). Further, latent variable modeling (such as forms of latent state-trait models; e.g., Geiser et al., 2017; Steyer et al., 2015) can be used to derive different scores (e.g., a latent trait, state, change score, etc.). Thus, density distribution parameters or latent scores could thus represent $\overline{\mathrm{P}}$ and $\overline{\mathrm{E}}$, respectively (e.g., the average of Ps, the standard deviation of Ps, the latent covariance shared among all Ps across all measurement occasions, etc.). Note here that the source of the data comes from repeated $\mathrm{P}$ and $\mathrm{E}$ measurements defined as momentary, state-like elements, but the resulting scores of $\overline{\mathrm{P}}$ and $\overline{\mathrm{E}}$ may also represent longer-term, trait-like elements depending on the parameters or models chosen. ${ }^{5}$ Theoretically, $\bar{p}$

\footnotetext{
${ }^{5}$ Some models, such as those from latent state-trait theory, decompose the observed variance in variables (here from repeatedly measured Ps and Es) into trait- and state-like components. Thus, sometimes $\overline{\mathrm{P}}$ and $\overline{\mathrm{E}}$ could represent both state- and trait-like entities.
} 
183 and $\overline{\mathrm{e}}$ could represent repeated measurements of $\mathrm{p}$ and e variables, respectively (e.g., in

184 longitudinal or measurement burst designs; Sliwinski, 2008), but it is not common to construct 185 density distributions around such enduring variables. Still, latent variable modeling may be used 186 to derive a latent mean or change score across time.

187 Third, $\dddot{\mathrm{P}}, \dddot{\mathrm{p}}, \dddot{\mathrm{E}}, \dddot{\mathrm{e}}$ represent person-oriented profile measurements where several variables 188 (measured at one time-point), as a profile, are nested within persons or environments. For $\dddot{\mathrm{P}}$ and 189 Ë, multiple different short-term person or environment variables constitute a profile for each 190 person or environment, respectively (or only one person or environment in an idiographic case 191 study design) at one single measurement occasion. For $\dddot{p}$ and $\dddot{e}$, multiple long-term person and 192 environment variables, respectively, constitute the profiles. Lastly, $\dddot{\overline{\mathrm{P}}}$ and $\dddot{\overline{\mathrm{E}}}$ are profiles of short193 term person and environment variables, respectively, that have been sampled repeatedly at 194 several measurement occasions. While profile elements may in fact be any kind of density 195 distribution parameters or latent scores, most likely aggregated values across measurement 196 occasions would be used for $\dddot{\overline{\mathrm{P}}}$ and $\dddot{\overline{\mathrm{E}}}$. Again, theoretically, $\dddot{\overline{\mathrm{p}}}$ and $\dddot{\overline{\mathrm{e}}}$ are also possible as profiles of 197 repeatedly measured and aggregated long-term person and environment variables, respectively; 198 but these are practically rarely if ever used. As explained previously, for all of these flections, 199 different data sources may have been used to measure the variables.

$200 \quad$ 2.1.3 Combinations. Equation 2 can be seen as the full four-element equation that 201 subsumes several truncated one-, two-, and three-element equations (see overview in Table 3). 202 For the four basic elements, there are thus 15 possible combinations. However, because $\mathbb{0}$ can 203 take the form of any of the four basic elements, there are actually 60 different combinations. 

multiple variables at once for each element and/or (2) multiple measurement occasions. First, in multiple-predictor models, several variables may have been simultaneously measured for the

208 same element. To give an example:

$$
\mathbb{O}=f\left(\mathbb{P}_{i}\right)=f\left(\mathbb{P}_{1}, \mathbb{P}_{2}, \mathbb{P}_{3}, \ldots, \mathbb{P}_{i}\right)
$$

Second, the variables may have been measured at different time-points, thus enabling time-sensitive analyses (see 4.4 Person-Environment Transactions). For example, a later (future) outcome variable at time $n$ may be predicted from concurrent variables also at time $n$ and prior 212 (past) variables at time $n$-1. To give an example:

$$
\mathbb{O}_{n}=f\left(\mathbb{P}_{n}, \mathbb{P}_{n-1}\right)
$$

\section{$213 \quad 2.2$ Functional Configurations}

The previous section on elements, flections, and combinations focused only on the person and environment ingredients of Equation 2 and how they could be measured and combined. However, their relations to an outcome variable as well as to each other - both within a given

217 time-point and across time-points - have not been touched upon so far. This relation question also concerns the exact nature and form of the function $f$. For example, functions can be additive,

219 multiplicative, proportional, or exponential, among others, but often the exact function relating

220 person and environment variables is left unspecified (Schneider, 2001, p. 145). The section 4.

221 Person-Environment Relations will concretize different functions that have been proposed in

222 extant personality, social, developmental, and industrial/organizational psychological literature.

223 Briefly, most research conceptualizes the function $f$ in linear and additive ways or in

224 multiplicative ways (see different effect patterns in 4.1 Person-Environment Interactions), and

225 some research also includes correlations (4.2 Person-Environment Correlations), fits (4.3.

226 Person-Environment Fit), and time-lagged analyses (see 4.4 Person-Environment Transactions). 


\section{A General Person-Environment Relations Model (PERM)}

Keeping the different ingredients and their functional configurations in mind, Figure 2 presents a formalized account of a generic and general Person-Environment Relations Model (PERM). As can be seen, several paths (a-t) representing different effects are distinguished in the PERM, which may additionally exist at several time-points (making it possible to examine their intra-individual stability and change as well as possible inter-individual differences therein). A summary of the effects and their meanings can be found in Table 4.

- Figure $2-$

\section{- Table $4-$}

Person variables may be associated with outcome variables ${ }^{6}$ (path a), as may environment variables (path b). However, both (as products) may also "interact" and thus have an interactive effect on an outcome, such that a person variable moderates the effect of an environment variable on an outcome variable (path $\mathrm{c}^{\prime}$ ) and also vice versa (path $\left.\mathrm{c}^{\prime \prime}\right)$. This effect is termed here a statistical person-environment interaction. Additionally, person and environment variables may be associated with each other concurrently (path d), which is termed here a person-environment correlation. This correlation (or individual differences therein) may, in turn, itself be associated with an outcome variable (path e). Person-environment correlations may exist due to different reasons (paths $\mathrm{f}^{\prime}, \mathrm{f}^{\prime}$ ), and these reasons are subsumed under the label of navigation mechanisms. Some of these mechanisms originate from the person and thus are tied to either $\mathbb{P}$ or $\mathbb{P}\left(\mathrm{path}_{\mathrm{g}}\right)$. Other mechanisms may, however, originate from or be tied to environment variables $\mathbb{E}$ or $\mathbb{e}$ (path g"). Additionally, mechanisms may also be directly associated with an outcome variable (paths h',

\footnotetext{
${ }^{6}$ Outcome variables could be sampled before, at the same time, or after the sampling of predictors (person and environment variables). If concurrently sampled, outcome variables cannot be seen temporally as consequences (for a nuanced discussion about consequences, see Wessels, Zimmermann, \& Leising, 2016). However, some variables are nonetheless considered "outcomes" in a conceptual sense and thus also distinguished from their predictors in a statistical modeling sense (e.g., when using regression analysis).
} 
$\mathrm{h}$ "). So far, all elements and their relations have been examined at one time-point of measurement (T1). However, there may be multiple time-points. The PERM in Figure 2 depicts another timepoint, T2. This allows also specifying stability effects between prior elements (T1) and later elements (T2) for navigation mechanisms (paths i', i"), person variables (path j), environment variables (path k), and outcome variables (path 1). Additionally, there may also be a stability of the person-environment correlation (path m). Further, there can be cross-lagged effects between person and environment variables, respectively, such that prior person variables predict later environment variables (path n) and prior environment variables later person variables (path o). This will be termed here a person-environment transaction. Prior outcome variables may also predict later navigation mechanisms (paths p', p"), person variables (path q), and environment variables (path r). Lastly, later person-environment correlations can also be predicted by prior outcomes (path s) and prior navigation mechanisms (paths t', t").

It is important to note that not all possible relations are depicted in the in the PERM (Figure 2) for simplicity (e.g., there are no direct or lagged paths from person-bound navigation mechanisms to the environment variables but only to the person-environment correlations). There may also be feedback (across time), self-organization and autopoiesis, and non-linear effect patterns (e.g., with quadratic or cubic effects), but these are not explicitly marked in Figure 2 and would need to be inferred from the exact $f$ functions when specified (e.g., using differential or polynomial equations). Further, relations among $\mathbb{P}$ with $\mathbb{P}$ and among $\mathbb{e}$ with $\mathbb{E}$, respectively, are also not depicted in Figure 2 but should also be considered. If subscribing to causal analysis, this could make it possible to distinguish six essential links between persons (i.e., with $\mathbb{P}$ and $\mathbb{P}$ ) and environments (i.e., with $巴$ with $\mathbb{E}$ ), most notably social ones, as outlined in Ryff (1987) and Pettigrew (1997). Specifically, (1) persons may influence specific situations (e.g., by selecting or changing one's situations; see 5.2.1), (2) specific situations may influence individuals (e.g., abuse 
as a traumatic experience; see 5.2.2), (3) situations may influence social structures (e.g., repeated interactions may lead to a certain social network), (4) social structures may influence situations (e.g., within a social network, several different interactions are possible), (5) individuals may influence social structures (e.g., one's social network could be dependent on one's personality; 5.2.1), and (6) social structures may influence individuals (e.g., one's social network may change one's personality via repeated interactions over time; 5.2.2). In general, all relations in Figure $2-$ including the ones not depicted for simplicity - are multi-determined, multi-functional, and constantly ongoing on short- and long-term timescales in a dynamic person-environment macrosystem. Thus, there are complex multivariate relations, some of which constitute moderations (paths c', c") and many mediations (e.g., path j may also flow via paths a and q). As such, the PERM already contains several hypotheses to be tested in future research. To what extent, when, why, or for whom the effects (paths) exist remain open empirical questions.

\section{Person-Environment Relations}

The PERM in Figure 2 contains four general person-environment relations:

1. Interactions (path $c^{\prime}$ or $c^{\prime \prime}$, keeping paths a and $b$ in mind)

2. Correlations (path d)

3. Fits (special cases involving interactions or correlations)

4. Transactions (paths $\mathrm{n}$ and $\mathrm{o}$ in conjunction)

Unfortunately, these terms have been used rather haphazardly or even synonymously throughout the literature. This is especially true for the term "person-situation interaction" which has come to be used as an umbrella term for different phenomena. The term has become conceptually blurry as it is currently being used at least in the following four ways (Lilienfeld et al., 2015; Olweus, 1977; Rauthmann, 2018a): (1) both persons and situations shape behavior together; (2) relations between person and situation variables are bidirectional (i.e., persons shape 
296

297

298

299

300

301

302

303

304

305

306

307

308

309

310

311

312

313

314

315

316

317

situations and situations shape persons); (3) persons and situations are interwoven in complex ways (i.e., their effects on behavior cannot be separated properly); and (4) statistical effects of person variables depend on situation variables and vice versa in the prediction of an outcome variable (i.e., moderation).

To gain conceptual and methodological clarity, it is paramount to clearly distinguish different person-environment relations and consistently use precise terminology to label them. To do so, this chapter maintains that (1) interactions concern how effects of person and environment variables on outcome variables depend on each other, (2) correlations concern how person and environment variables are associated concurrently, (3) fits concern the matching of person and environment variables to each other, and (4) transactions concern how person and environment variables are related ${ }^{7}$ to each other across time. It will also be shown how person-environment fit can be captured either by attending to certain effect patterns (which include person-environment interactions) or to person-environment correlations. As such, interactions and correlations can also signify fit in certain instances.

\subsection{Person-Environment Interactions}

The idea of an interaction between person and environment variables (the latter being historically mostly operationalized as "situation" variables) in predicting outcome variables of interest was born out of a consequence of the person-situation debate. The person-situation debate - which can be considered resolved today - has revolved around several issues (Fleeson \& Noftle, 2008a,b; Kenrick \& Funder, 1988). A notable idea was that the level, consistency, or stability of behavior was more strongly (or even solely) determined by environmental variables (such as the cues or characteristics of situations) than by person variables (such as personality

\footnotetext{
${ }^{7}$ The PERM is currently phrased to be rather agnostic about whether these across-time-relations should represent causal explanations or mere predictions (but see 6.4 Explanation vs. Prediction and Causality). This will depend on the framework and the design within which a study on those relations is conducted.
} 
traits). This resulted in efforts that pitted the person and the environment (situation) against each other. Consequently, an implicit assumption was that person and environment variables predicted outcomes independently of each other and that the prediction from person variables would be negligible. As a counter to such a simplistic understanding, interactional psychology (Ekehammar, 1974; Endler \& Magnusson, 1976; Magnusson \& Endler, 1977a,b) maintained that there can also be an interaction between person and environment variables. That interaction has often been interpreted in statistical terms such that (after controlling for the respective main effects of both the person and environment variables) the effect of the person variable on an outcome variable depends on the environmental variable and, vice versa, the effect of the environment variable on the outcome variable depends on the person variable. Statistically, this describes a moderation effect (see paths c' and c" in Figure 2). Conceptually, it means that persons with certain levels on their person variables respond differently to (or show different levels of outcome variables in) environments with certain levels on their environment variables. Conversely, it also means that environments with certain levels on their environment variables elicit different responses for persons with certain levels on their person variables. This basic notion of an interaction can also be found in educational (aptitude-by-treatment interactions: ATI; e.g., Snow, 1989), applied (Trait Activation Theory: TAT; e.g., Tett et al., 2013), clinical (diathesis-stress models; e.g., Zuckerman, 1999), and behavior genetic literatures (geneenvironment interactions; e.g., Briley, 2020; Caspi et al., 2002; Johnson, 2007).

In the available literature dealing with statistical interactions between person and environment variables, there are actually three distinct strands of interactionist research: (1) variance decomposition, (2) linear interaction, and (3) non-linear approaches. However, these three can be seen as special cases of (4) more general effect patterns when predicting outcomes from person and environment variables, which also subsume (5) an outcome-based, variable- 
342 oriented definition of person-environment fit. The following paragraphs give an overview of 343 these five strands where interactions are key.

344 4.1.1 Analysis of Variance. The variance decomposition approach examines variance 345 associated with persons, situations, and person $\times$ situation interactions in ANOVA-type designs 346 (Bowers, 1973; Furr, Chapter 28). To do so, a set of persons needs to be exposed to a set of 347 situations, and their experiences (e.g., perceptions, affect) and/or behavior need to be recorded. 348 This creates a design where persons and situations are fully crossed and variance in dependent or 349 outcome variables (e.g., perceptions, behavioral responses) can be decomposed into different 350 sources: persons, situations, and person $\times$ situation interactions (mixed with error if multiple 351 items have not been used to measure the outcome variable). An example of this line of thought is 352 the anxiety S-R inventory from Endler and Hunt (1966) where persons indicate how anxiously 353 they would react $(\mathrm{R})$ to a set of situations $(\mathrm{S})$. Variance in anxious reactions can then be 354 decomposed into stemming from the two main effects of persons (= inter-individual differences

355 between persons in generally reacting anxiously) and situations (= inter-situational differences

356 between situations in their general tendency to elicit anxious reactions), plus the two-way

357 interaction of person $\times$ situation (= uniquely anxious reactions of specific persons to specific

358 situations, beyond the two main effects). A typical finding of variance decomposition research is 359 that the person $\times$ situation interaction is quite sizable (Lakey, 2016). However, measurement 360 error has often not been taken into account (which is often confounded with interaction variance;

361 Kenny, 1994; Rauthmann \& Sherman, 2019), and estimates of explained, reliable variance

362 depend crucially on the selection of persons, situations, and reactions as well as the methods used 363 to assess and analyze them (Cooper \& Withey, 2009; Geiser et al., 2015; Judd, Westfall, \& 364 Kenny, 2012). 
366 in a monolithic fashion, when in fact there can be different forms or shapes of interaction

367 (Widaman et al., 2012), as shown in Figure 3. For example, at least ordinal interactions (e.g., in

368 the diathesis-stress model; Zuckerman, 1999) and disordinal interactions (e.g., in the differential-

369 susceptibility model: Ellis et al., 2011) should be distinguished. For example, in the ordinal

370 interaction form as hypothesized by the diathesis-stress model (upper left side of Figure 3), the

371 quality of environments only plays a role for at-risk persons (e.g., with genetic vulnerability)

372 whose outcomes are worse in "bad"8 environments. In contrast, resilient persons seem

373 impervious to the effects of environments. Further, "good" environments confer no differential

374 benefits to either resilient or at-risk persons. In the disordinal interaction form as hypothesized by

375 the differential-susceptibility model (lower left side of Figure 3), malleable persons profit

376 strongly from "good" environments, but also suffer greatly from "bad” ones. In contrast, non-

377 malleable persons are again impervious to effects of environments. Thus, the malleable persons

378 will show worse or better outcomes, respectively, than the non-malleable persons.

Figure 3 additionally shows on the right side some other hypothetical interaction forms.

380 These could each be interpreted from the perspective of either persons or environments,

381 depending on what units of analysis researchers are interested in. For example, a linear

382 synergistic interaction approach considers how person and environment variables "work

383 together" (synergy) in a linear way to explain an outcome variable. A "full linear crossing" (a

384 disordinal interaction) is also displayed. The type of interaction captured is one of amplification

385 (or if viewed conversely, of suppression or attenuation): the effect of a person variable on an

\footnotetext{
${ }^{8}$ Environments (and also outcomes) are of course not per se "good" or "bad." Rather, some environments are more, and others less conducive with regard to a predicting a certain (often socially desirable or undesirable) outcome. What is good" or "bad" may thus depend on the research, cultural, and historical context.
} 
outcome is amplified (or attenuated) by an environment variable. This idea has been applied to different affective domains (e.g., anxiety: Endler, 1975; anger: Spielberger, 1988) and justice behavior (Schmitt, Eid, \& Maes, 2003; Schmitt \& Sabbagh, 2004).

- Figure 3 -

Most conceptualizations and studies examining linear interactions have focused on the following version:

$$
\mathrm{P}=f(\mathrm{p}, \mathrm{E})=\mathrm{p}+\mathrm{E}+\mathrm{p} \times \mathrm{E}(+ \text { residual error })
$$

Equation 5

To give an example, persons with higher levels on p (e.g., trait anxiety) show a higher increase in their levels of $\mathrm{P}$ (e.g., intensity of anxious feelings or behaviorally expressed fear) from lower to higher levels of E (e.g., quantity and/or quality of anxiety-inducing stimuli) than do persons with lower levels on $\mathrm{p}$ who show a smaller increase in $\mathrm{P}$ levels. This means that persons with higher $\mathrm{p}$ levels are more attuned or differentially sensitive to E levels than persons with lower $\mathrm{p}$ levels. In other words, $\mathrm{p}$ amplifies the effect of $\mathrm{E}$ on $\mathrm{P}$ in this example. Alternatively, one may also flip this interpretation and state that $\mathrm{E}$ amplifies the effect of $\mathrm{p}$ on $\mathrm{P}$ (this would be an environment perspective). While conceptually and statistically straightforward, this strictly linear interpretation of an interaction suffers from unrealistic assumptions, most notably that the level of an outcome, such as behavior, could increase (or decrease) infinitely (which is not possible as there are natural or measurement-related upper and lower boundaries of most variables).

4.1.3 Non-linear Effects. As a remedy of issues associated with linear interactions, more recent non-linear approaches have been proposed (e.g., Traits as Situational Sensitivities = TASS: Marshall \& Brown, 2007; Nonlinear Interaction between Person and Situation = NIPS: Blum et al., 2018; Schmitt et al., 2013). For example, the NIPS model posits that the non-linear effect follows the shape of a logistic regression which is achieved because both persons and environments can be either "weak" or "strong" (see last panel on the right side of Figure 3; e.g., 
Blum et al., 2018; Cooper \& Withey, 2009; Dalal et al., 2014; Leising \& Müller-Plath, 2009; Meyer, Kelly, \& Bowling, 2020; Meyer \& Dalal, 2009; Meyer, Dalal, \& Hermida, 2010; Schmitt et al., 2013). There are different ways to conceptualize the strength of a situation or environment (Rauthmann, 2018b; for a review, see Meyer et al., 2020). However, one way is to surmise that strong environments have extreme scores on their variables (e.g., extremely anxiety-inducing vs. extremely calming) where inter-individual differences between persons matter less to the manifestation of some outcome such as behavior (i.e., variance between persons in their reactions or reaction levels is restricted). This would mean that persons with different trait anxiety levels would not show pronounced differences in their behavioral displays in strong environments (i.e., more trait-anxious and less trait-anxious persons react with similar amounts of fear or calmness, respectively). Thus, environment variables can, under such circumstances, have more power over an outcome than person variables. In contrast, weak environments have moderate scores on their variables where individual differences in the outcomes manifest more readily and thus become salient. This would mean that persons with different trait anxiety levels would show corresponding differences in their behavioral displays (i.e., more trait-anxious persons will react with more fear than less trait-anxious persons). Thus, person variables can, under such circumstances, have more power over an outcome than environment variables.

The preceding ideas pertain only to the environment side, but they could also be applied to the person side. Thus, in a similar vein, there can be "strong" persons with extreme values on a trait (e.g., extremely low vs. extremely high trait anxiety) that react in certain trait-corresponding ways (e.g., with fear or calmness) regardless of the levels of environment variables. Thus, under such circumstances, their traits may matter more than the environment in the prediction of an outcome. In contrast, "weak" persons do not show extreme trait values, and thus the levels of the environment variable will be stronger predictors of their behavior. Hence, under such circumstances, the environment may matter more than the traits in the prediction of an outcome. 
Combining the idea of weak vs. strong environments and persons, respectively, necessarily yields non-linear effects on outcomes which have already been empirically tested for circumscribed construct domains (e.g., aggression and jealousy: Blum et al., 2018). An example is given in Figure 3 (bottom panel), which can be seen from the perspective of either person or of environment variables. However, the general operation of the NIPS model (which makes far more assumptions than just non-linear effects), specific instantiations of non-linear interactions, and the generalizability to other construct domains remain to be examined. Further, parts of the NIPS model (i.e., non-linear effects of person and environment variables) can also be situated within different effect patterns (see next section, 4.1.4 Effect Patterns) that themselves may be viewed as specific instances of a full, second-order polynomial regression.

4.1.4 Effect Patterns. Given the possible linear and non-linear effects of person and environment variables on outcomes (see paths a, b, and c'/c" in Figure 2), different effect patterns can be distinguished. In roaming the literature (see Krueger, 2009), three historically consecutive statistical perspectives on effect patterns can be identified (which are, of course, also based on or informed by theoretical considerations): Linear, interactionistic, and non-linear perspectives. Of these, the linear and some of the interactionistic patterns have already been empirically examined, while the non-linear patterns rarely receive attention and have yet to be examined in full detail. Each of these perspectives, and the different positions encapsulated within each of them, entails different ramifications for how the function $f$ relating person and environment variables to outcome variables is specified. The perspectives are compiled in Table 5, and the prototypical prediction patterns or effects they would produce can be found in Figures $4 a-c$. These figures thus demonstrate which shape person-environment relations could take in the prediction of outcomes. Notably, a variable-oriented perspective is employed here where effects pertain to certain variables of interest across sampled persons and/or environments, respectively. 
First, four linear patterns can be distinguished (see Table 5, Figure 4a): Zero (neither the person nor the environment variable predict the outcome), Person-only (only the person variable predicts the outcome), Environment-only (only the environment variable predicts the outcome), and Additive (both the person and the environment variable predict the outcome independently). Second, another four interactionistic patterns can be distinguished (see Table 5, Figure 4b): Interaction-only (only the interaction effect of the person and environment variable predicts the outcome), Person plus Interaction (the person variable as well as the interaction between the person and environment variable predict the outcome), Environment plus Interaction (the environment variable as well as the interaction between the person and environment variable predict the outcome), and Additive plus Interaction (the person variable, the environment variable, and the interaction between the person and environment variable predict the outcome). Though any of the previously summarized eight patterns (linear and interactionistic, respectively) could be equipped with quadratic effects of person and environment variables, respectively, only four "extremes" of non-linear patterns are presented here (see Table 5, Figure 4c): Person Strength (only the quadratic person variable predicts the outcome), Environment Strength (only the quadratic environment variable predicts the outcome), Non-linear Additive (both the quadratic person variable and quadratic environment variable predict the outcome independently of each other), and Non-linear Additive plus Interaction (the quadratic person variable, quadratic environment variable, and the interaction of the person and environment variable predict the outcome).

The 12 overly simplified effect patterns outlined here are just a selection, as with five predictors (i.e., two main effects of person and environment variables, their respective quadratic effects, and their interaction effect) actually 30 distinct effect patterns are possible if at least one 
483 or maximally five predictors actually predict an outcome. However, to conserve space and only

484 show general tendencies, the 12 most important, interesting, or salient patterns have been

485 compiled in Table 5 and Figures 4a-c. These can be contrasted against another effect pattern,

486 termed Full Complexity here, that is essentially an unconstrained, full second-degree polynomial

487 regression equation where all predictors meaningfully predict the outcome (Edwards, 2002). For

488 example, if we used as predictors a short-term person (P) and a short-term environment variable

489 (E) to predict a momentary outcome variable (0), then we would use following equation:

$$
\mathrm{O}=f(\mathrm{P}, \mathrm{E})=\mathrm{P}+\mathrm{E}+\mathrm{P}^{2}+\mathrm{P} \times \mathrm{E}+\mathrm{E}^{2} \quad \text { Equation } 6
$$

Figure $4 d$ contains two examples for such a Full Complexity effect pattern where the regression coefficients of all predictors are statistically significant or sizable. Specifically, the full polynomial regression in Equation 6 predicts an outcome variable simultaneously from a person variable $\left(b_{1}\right)$, an environment variable $\left(b_{2}\right)$, the square of the person variable $\left(b_{3}\right)$, the person variable $\times$ environment variable interaction $\left(b_{4}\right)$, and the square of the environment variable $\left(b_{5}\right)$.

The resulting regression weights $b_{1-5}$ can, in turn, be used to compute four response surface parameters $a_{1-4}$ that describe a three-dimensional plot (Box \& Draper, 1987; Edwards, 2002; Edwards \& Parry, 1993), facilitate visual inspection and understanding of effect patterns, and 498 allow the testing of nuanced hypotheses (Humberg et al., 2019; Schönbrodt, 2016a,b).

499 Confidence intervals and inferential statistics with $p$-values are also available for these response 500 surface parameters. Polynomial regression and response surface analyses allow testing quite nuanced hypotheses, most notably pertaining to fit (see 4.1.5 Outcome-based, Variable-oriented

502 Person-Environment Fit). Further details on polynomial regression and response surface analysis 503 can be found in Box 1. 


\subsubsection{Outcome-based, Variable-oriented Person-Environment Fit. Polynomial}

regression models and subsequent response surface analyses have been used to understand outcome-based person-environment fit (e.g., Edwards, 2002, 2008; Edwards \& Shipp, 2007;

Edwards et al., 1998). "Outcome-based" means that "fit" between predictor variables is defined here always in terms of predicting an outcome, and hence a "fit" may mean different things for different outcomes even if the same predictors are used in the same dataset. Roughly, there are two operationalizations of such outcome-based fit. First, there are strict and lax patterns of fit that can be identified from the polynomial regression and response surface parameters (see Humberg et al., 2019 for six conditions that need to be met to conclude an actual fit pattern). Three such patterns are presented exemplarily in Figure 4d. Most notably, these fit patterns all contain nonlinear terms - quadratic effects and interactions. Interactions thus are key to this kind of fit.

Second, more pragmatically, person-environment fit could also be operationalized as the local "optimum level of outcome achieved" at a certain combination of measured values of the person and environment variable. For example, though not numerically congruent, the combination of the scores 2.3 on the person variable and 4.1 on the environment variable (both on a scale from 1 to 5) may together yield the highest scores on the outcome variable. In other words, it is at 2.3 and 4.1 when the person and environment "fit" to have produced the highest levels of an outcome. ${ }^{9}$ Such an interpretation of fit, however, begs the question whether any (optimal) prediction of an outcome by person and environment variables could be considered person-environment fit. On the one hand, proponents of the commensurability idea (e.g., Caplan, 1987) would require the person and environment variables to be "similarly measured" in terms of

\footnotetext{
${ }^{9}$ In principle, one could also be interested in the lowest or any other most valuable level of an outcome. What "fit" means is always outcome-dependent here, and the desirability of the outcome variable (e.g., lifesatisfaction, depression, salary, unemployment, divorce) then plays a crucial role in how we approach and interpret person-environment fit.
} 
content (e.g., person variable: sociability; environment variable: sociality) and graded response scales $^{10}$ - only then would it be sensible to examine fit. On the other hand, others (e.g., Kristof, 1996) have taken a laxer approach where certain forms of fit (e.g., supplementary vs.

complementary: Cable \& Edwards, 2004; Muchinsky \& Monahan, 1987) may require more commensurability and others less (for details, see 4.3 Person-Environment Fit).

Outcome-based person-environment fit is variable-oriented and defined as a pattern of effects (Humberg et al., 2019) - as opposed to person-environment correlations that can be examined in a person-oriented manner and have also been conceptualized as fits (see 4.2 PersonEnvironment Correlations). Usually, no individual differences in this kind of fit can be derived (but see Nestler, Humberg, \& Schönbrodt, 2019) ${ }^{11}$, which also means that typically no nomological network is cast around it to examine its correlates (e.g., causes/antecedents, concomitants, and consequences). Further, also no (inter-individual differences in intraindividual) stability and change can be established. ${ }^{12}$ However, this form of fit still allows testing quite nuanced hypotheses for how an outcome variable is predicted conjointly by person and environment variables. Notably, it has been repeatedly emphasized that these kinds of analyses can only yield meaningful findings and actually test the nuanced hypotheses when the person and environment variables are conceptually, methodologically, and numerically commensurate (Schönbrodt, 2016a,b). If this is not the case, then the hypotheses cannot be tested directly, but the patterns of effects (i.e., the regression coefficients in the full polynomial regression) and the

\footnotetext{
${ }^{10}$ The mere fact that the same response scale (e.g., from 1 to 5) has been used would likely not suffice to conclude strong commensurability. For example, a "3" may mean different things on a trait scale for sociability and a situation scale for sociality despite perfect numerical commensurability. Their scalability likely differs.

${ }^{11}$ An exception would be multi-level polynomial regressions and response surface analyses where person, environment, and outcome variables at level 1 are nested within persons at level 2 . In such a case, variance in slopes and also response surface parameters could be examined as individual differences. For details, see Nestler et al. (2019).

${ }^{12}$ It is still possible to quantify the stability of the response surface parameters $a_{1-4}$ across time-time points (essentially asking whether they remain the same across time).
} 
overall shape of the response surface may still be revealing and of interest; however, strong caution is warranted with fit interpretations and conclusions. For example, such a cautious approach has been used in personality and social-psychological research to understand the relations of income with the fit between personality traits and job demands (Denissen et al., 2019); of self-esteem with the fit between one's own personality traits and those from one's home city (Bleidorn et al., 2016); of perspective-taking with the fit between one's own and one's community's ideological orientations (Chopik \& Motyl, 2016); and of peer-victimization with the (mis-)fit between one's own personality traits and those from one's peer group (Boele et al., 2017). More complex analyses have also examined moderators, such as the study of Ilmarinen et al. (2017) where friendship homophily in traits and cognitive abilities (i.e., persons with similar personalities nominate each other as friends) was examined as a function of classroom sizes and children's gender. Further, polynomial regressions and response surface analyses have been used in numerous industrial/organizational and work-psychological studies examining person-team, person-job, and person-organization fit, among others (e.g., Edwards \& Shipp, 2007).

4.1.6 Summary and Findings. What is the evidence for the occurrence, size, and shape of person-environment interactions? The answer to this question crucially depends on how the person and environment variables are defined and operationalized as well as which research design is used. For example, variance decomposition approaches usually find sizable interaction effects (Lakey, 2016; see also Furr, Chapter 28). In contrast, there seem to be barely any consensually agreed upon and replicable molecular gene $\times$ environment interaction effects (Briley et al., 2019; cf. Caspi et al., 2002). However, gene-environment interactions remain popular in behavior genetics (Manuck \& McCaffery, 2014), with distinct patterns of effects proposed (Belsky \& Pluess, 2009; Briley, 2020; Roisman et al., 2012), such as diathesis-stress (genetic predispositions only manifest under certain environments), vantage sensitivity 
570 (environments override genetic predispositions in phenotypic manifestation; removing them leads

571 to genetic differences gaining influence), and differential susceptibility (some persons are

572 specifically attuned to and others immune to certain environmental influences). There seems to

573 be some (replicable) evidence for diathesis-stress and vantage sensitivity effects, but less for

574 differential susceptibility (see Briley, 2020). Further, in work contexts, interactions between traits

575 (p) and organizational or work variables (mostly e) have been found repeatedly and noted to be

576 substantial and meaningful (for a cursory overview of industrial/organization-psychological

577 studies, see Table 6.2 in Tett et al., 2013, pp. 93-94). In contrast, however, experience sampling

578 data in daily life have suggested that interaction effects between personality traits (p) and

579 momentarily perceived situation characteristics $(\mathrm{E})$ predicting momentary personality states $(\mathrm{P})$

580 were very small, if they could be detected at all (Sherman et al., 2015; cf. Breil et al., 2019).

581 More recently, Sherman and Pashler (2019) estimated the magnitude of interactions between

582 person and environment variables in five studies, with 42 to 542,700 effects per study. The

583 averaged incremental $R$ s (computed across all possible models within each study) beyond the

584 main effects of the person and environment variables ranged from .019 to .131 for those five

585 studies. These effect sizes thus seem rather miniscule although even supposedly small effects can

586 be practically meaningful, consequential, or accumulate across time (Funder \& Ozer, 2019).

587 These disparate findings can be reconciled when considering several issues. First, person-

588 environment interactions derived from experience sampling data in daily life may look differently

589 than those from experimental designs. Specifically, the signal to noise ratio may be different

590 (with more noise in repeatedly measured field data) so that interactions - which are notoriously

591 subtler than main effects - may be more difficult to detect. Second, detecting interaction effects

592 reliably not only requires adequately powered studies but also a careful (e.g., representative)

593 selection of persons and environments. Violating these principles may lead to Type I and II errors 
594 (i.e., either spuriously finding interactions or missing actual interactions). Third, and associated

595 with the previous point, researchers need to attend to balances and symmetries in their data and 596 predictive models (see Table 3). For example, examining $\mathrm{P}=f(\mathrm{p}, \mathrm{E})$ may be balanced (there is 597 one $\mathrm{p}$ and one E predictor), but not symmetrical (a short-term $\mathrm{P}$ is supposed to be predicted from 598 a long-term $\mathrm{p}$ with a short-term $\mathrm{E})$. Given that there likely is covariance shared among $\mathbb{P}, \mathbb{P}, \mathbb{E}$, 599 and e (see 4.2 Person-Environment Correlations) and that said covariance may be differentially 600 distributed according to short- vs. long-term timescales, attending to balances and symmetry is 601 important. For example, short-term $\mathbb{P}$ and $\mathbb{E}$ may be differently correlated to each other than long602 term $\mathbb{P}$ and $\mathbb{e}$, and those time-scale symmetrical correlations may differ from asymmetrical ones 603 such as $\mathbb{P}$ with $\mathbb{e}$ and $\mathbb{E}$ with $\mathbb{P}$. Fourth, the type of measurement of both person and environment 604 variables - especially with which data sources they are tapped (e.g., self-ratings of P and in situ 605 ratings of E) - may also account for the differences observed in the literature. Lastly, by focusing

606

607

608

609

610

611

612

613

614

615 616 only on linear interactions, potentially interesting and important patterns in the data may be missed. Specifically, non-linear terms may yield a fuller picture and even allow examining nuanced hypotheses about person-environment fit in relation to outcomes variables (see 4.1.4 Effect Patterns and 4.1.5 Outcome-based, Variable-oriented Person-Environment Fit).

\subsection{Person-Environment Correlations}

A downstream net result of the many effects and transactions going on in the PERM is the correlation between person and environment variables. This means that certain persons are nonrandomly assorted into certain social and non-social environments. Table 6 compiles 32 different forms of person-environment correlations that are possible depending on which person and environment variables or profiles serve as input (for options, see Table 2).

- Table $6-$ 
As can be seen in Table 6, a broad distinction can be made between (1) variable-oriented

$618 R$ correlations (e.g., a single person and a single environment variable are correlated across a

619 person sample) and (2) person- or profile-oriented $Q$ correlations (e.g., a profile of person

620 variables is correlated with a corresponding profile of environment variables within single

621 persons). Another important distinction concerns (3) the symmetry regarding the person and

622 environment variables' respective timescales. For example, a PE correlation is symmetrical (both

623 the person and environment variables are defined as short-term variables; e.g., Rauthmann et al.,

624 2016), while a $\mathrm{pE}$ correlation is not (one variable is defined as long-term, the other as short-term;

625 e.g., Rauthmann et al., 2015c). Lastly, a person-environment correlation can be interpreted

626 simply as a meaningful or statistically significant association between a person and environment

627 variable or as person-environment fit. The latter is a special interpretation of person-environment

628 correlations, and some may argue it is only warranted when there is commensurability between

629 the person and environment variables (i.e., at least in terms of content, measurement, and

630 scalability; see also 4.1.5 Effect Patterns, 4.1.5 Outcome-based, Variable-oriented Person-

631 Environment Fit, and 4.3 Person-Environment Fit).

632 4.2.1 Variable-oriented Correlations. If persons and environments are non-randomly

633 intertwined, then single person and environment variables should be correlated to some extent.

634 Such correlations, if statistically significant, sizable, or meaningful, can be a result of ongoing

635 transactions or, more specifically, how persons find themselves in and navigate their

636 environments (see 5. Person-Environment Calibrations and Navigation Mechanisms). There are

637 several reasons why single person and environment variables may become associated or

638 synchronized across time. For example, one such reason is that persons, to a certain extent, select,

639 evoke, modify, and create their environments. If this happens in person-congruent ways (e.g.,

640 according to one's traits or to one's momentary goals), then person and environment variables 
641 will show correlations to some extent (Fraley \& Roberts, 2005; Ickes et al., 1997). This may be

642 the case for both symmetrical (two long-term or two short-term variables being correlated) and

643 asymmetrical correlations (one short-term and one long-term variable being correlated).

Details on the quantification of variable-oriented person-environment correlations can be

645 found in Box 2. The following is a necessarily eclectic snapshot of a myriad of studies that have

646 quantified concurrent person-environment correlations. However, studies from the six domains of

647 (1) behavior genetics, (2) work, (3) relationships, (4) social investments, (5) daily life, and (6)

648 simulations are singled out here because they showcase a broad range of topics studied and were 649 substantively interested in person-environment correlations.

650

651

652

653

654

655

656

657

658

659

660

661

662

663

664
- Box 2 -

4.2.1.1 Behavior Genetics. The behavior-genetic literature has already catalogued ways in which persons with a certain set of genes (p) are non-randomly found in certain environments or niches (e) (Kandler et al., Chapter 7; Plomin, DeFries, \& Lohelin, 1977; Scarr \& McCartney, 1983). Such gene-environment correlations are non-negligible as they have implications for estimates of specific model parameters in behavior-genetic analyses (Briley et al., 2019), such as the analysis of twin data (Purcell, 2002). An interesting downstream effect of genotypeenvironment correlations (i.e., two correlated long-term variables) is that measures of the environment (e.g., self-perceptions, informant-perceptions, and observations) also show genetic contributions (7-39\%; Kendler \& Baker, 2007). In this vein, Plomin et al. (1990) have also found genetic influences on life events, which may be mediated by personality traits (Saudino et al., 1997). This means that even the environments we find ourselves in likely are, at least partly, also driven by our genotypes and personalities. This nicely exemplifies what person-environment correlations conceptually capture: The person and the environment are inextricably concatenated in complex ways which means that person variables will contain variance from environments and 
665 environment variables variance from persons - even if person and environment variables can be 666 theoretically and even measurement-wise neatly segregated.

667

668

669

670

671

672

673

674

675 676 jobs.

677

678

679

680

681

682

683

4.2.1.2 Work. For demands in work environments, Denissen et al. (2014) examined, among other things, for each of the Big Five domains how self-reported personality trait scores (p) of persons working in certain vocations were correlated with personality-related role demands (e) of those vocations (as rated by students and experts). They termed this person-environment correlation the "kernel of truth" because it conveys that "occupations that score high on a particular personality norm demand are also carried out by persons who actually score high on the corresponding trait" (p. 1936). Those correlations ranged from -.14 (conscientiousness) to .69 (openness) and were particularly high for openness, extraversion, and agreeableness. Thus, on average, persons reported trait levels that were concordant with consensual role demands of their

For stressors in work environments, Ford et al. (2014) conducted a meta-analysis on synchronous (same-time) stressor-strain effects, where "stressors" are environmental variables (e.g., workload, conflicts) and "strain” person variables (e.g., psychological and physical symptoms, such as exhaustion, irritation, depression, illness). They used studies that measured stressors and strains at least at two time-points so they could examine to what extent stressorstrain correlations increased across time. Stressors were correlated meta-analytically with psychological strain at .35 (Time 1) and .37 (Time 2) and with physical strain at .12 (Time 1) and .14 (Time 2).

4.2.1.3 Relationships. Turning to social environments, one could view assortative mating and friendship homogeneity effects as person-environment correlations. For example, if trait levels of friends and/or romantic partners are similar to persons' own trait levels, then such person-person similarity indexes person-environment correlations. In such cases, the environment is defined as other persons, their behaviors, their personalities, or the relationships or 
690 interpersonal dynamics with them (Asendorpf, 2020; Back, Chapter 8; Clark, Lemay, \& Reis,

691 2020; Roche \& Cain, Chapter 25; Pincus et al., 2020; Molho \& Balliet, 2020).

692 A recent study with several thousand Facebook users examined assortative person-

693 environment correlations for friendships and romantic partners using status updates and likes

694 which were algorithmically converted to Big Five scores (Youyou et al., 2017). Additionally, the

695 authors had self-reports on the Big Five and thus were able to also quantify self-report-based

696 similarities. In all instances, similarities (expressed in $r$ units) were - on average across all Big

697 Five traits - rather small to moderate for self-reported scores, likes-derived scores, and language-

698 derived scores from status updates for friends $(.06, .14, .24)$ and couples $(.10, .24, .38)$. Thus, this

699 research demonstrates that there is some, though likely small non-random assortment among

700 persons (i.e., personality homophily: correlations between a person's trait levels and others' trait

701 levels). The evidence for the implications and consequences of such personality homophily seems

702 to be mixed, however. For example, Weidmann et al. (2017) used concurrent and longitudinal

703 dyadic polynomial regression analyses and found only little evidence that the similarity of

704 heterosexual couples in their Big Five traits conferred benefits for relationship satisfaction.

705 Whether persons select others who are more similar to themselves (niche selection or

706 construction) and/or actually become similar to each other across time (e.g., via social influences)

707 is often not disentangled. However, recent studies showed that friendship similarity seems to be

708 mainly driven by niche construction mechanisms (Bahns et al., 2017). Such questions concern in

709 fact dynamic person-environment transactions (see 4.4) as well as calibrations and navigation

710 mechanisms (see 5.). Person-environment correlations, as presented here, are defined to be non-

711 dynamic and stationary but dynamic mechanisms can explain their occurrence.

712 4.2.1.4 Social Investments. Looking at social investments into different life domains,

713 experiences, and social roles, Lodi-Smith and Roberts (2007) conducted a meta-analysis on the 
714 relations between the three "maturity" Big Five traits (agreeableness, conscientiousness,

715 emotional stability) and social investments into work, family, religion, and volunteerism in cross-

716 sectional studies. These findings can thus be relevant to person-environment correlations insofar

717 as actual environment and not person variables (e.g., experiences, performance, behaviors) have

718 been sampled in the studies that went into the meta-analysis. Meta-analytical correlations

719 between the three maturity traits and social investment categories ranged from .11 to .19 for

720 work, from .15 to .16 for family, from .00 to .16 for religion, and from .02 to .15 for

721 volunteerism. Together, the meta-analysis provided evidence for small to moderate relations

722 between mature traits and social investments in different life domains.

723 4.1.5 Daily Life. The previous studies all concerned pe correlations between enduring

724 variables, but what about PE correlations between momentary variables? For example, Fleeson

725 (2007) found contingencies between Big Five state enactments and momentary situation

726 characteristics (anonymity, task orientation, others' friendliness, others' status) to range between

$727-0.10$ to 0.67 in his Study 1 and between -0.18 to 0.30 in his Study $2^{13}$, with some inter-individual

728 differences in those contingencies $(S D \mathrm{~s}=0.07-0.25$ in Study 1 and $S D \mathrm{~s}=0.10-0.20$ in Study 2).

729 Sherman et al. (2015) and Rauthmann et al. (2016) examined in self-reported experience

730 sampling data, among other things, concurrent associations between 10 personality states (P; Big

731 Five states, plus honesty/humility, dominance, self-esteem, happiness, and authenticity) and 8

732 momentary experiences of situation characteristics (E; DIAMONDS: Rauthmann et al., 2014).

733 On average across all possible 80 pairings of person and environment variables (Rauthmann et

734 al., 2016), absolute cross-sectional associations amounted to $0.09^{13}(S D=0.08)$. Looking at only

73523 pairings that were a priori specified as conceptually plausible and sensible, the average

\footnotetext{
13 As with most multi-level model analyses, these are unstandardized effects. This means: For every one-point increase in the predictor, the criterion increases in the amount of the unstandardized effect.
} 
736 absolute effect size was higher at $0.16(S D=0.07)$. It seems that when it comes to more short-

737 term timescales (i.e., hours in the case of experience sampling methodology), then momentary

738 person-environment correlations may be smaller. However, even if they are small momentarily,

739 they could accumulate to impressive effects across longer periods of time (see Funder \& Ozer,

7402019 for a discussion). Furthermore, Sherman et al. (2015) also estimated the amount of inter-

741 individual differences in contingencies (ranging from 0.08 to 0.26 ) which were barely related to

742 personality traits (i.e., only small and often statistically non-significant cross-level interaction

743 effects in multi-level modeling). Thus, taken together, there appear to be small to moderate

744 actualgenetic person-environment correlations ("contingencies") but their nomological networks

745 are poorly understood so far. The surveyed findings await replication, and person-environment

746 correlations deserve a more systematic and broader examination.

4.2.1.6 Simulations. Using computational modeling with simulations, Fraley and Roberts (2005) examined person-environment relations across ages. In their model, the correlation between individual differences in a psychological or person variable and an environment variable increased sharply from age 0 to 7 and then reached a plateau of approx. $r \approx .35$ around age 8 and later (see their Figure 4 on p. 69). They termed this correlation "coherence" (for a full overview on this term, see Fournier, Chapter 17) and linked it to person-environment fit. However, whether or not a variable-oriented person-environment correlation represents "fit" is likely more a conceptual question. Regardless, it is interesting that this finding reflects the empirical personenvironment correlations cursorily reviewed above relatively well. This additionally lends

756 credence to Fraley and Robert's (2005) model and its operationalizations. 
760 391) defines fit as "a state of compatibility of joint values of one or more attributes, a, b, c, . . j,

761 of a focal entity $(\mathrm{P})$, and a commensurate set of attribute values, a, b, c, . . j, of the entity's

762 environment (E)". This definition can be expanded to a profile approach which, according to

763 O’Reilly, Chatman, and Caldwell (1991), “(...) comprehensively assesses individuals and

764 situations using a common language, allows for the ipsative measurement of individual

765 characteristics by arraying attributes in terms of their salience to the individual, and provides a

766 direct measure of person-situation fit" (p. 491). Empirical research has already adopted such a

767 profile approach to fits between enduring $\dddot{p}$ and $\dddot{e}$ profiles (e.g., need-reinforcer correspondence:

768 Rounds, Dawis, \& Lofquist, 1987; person-job fit: Caldwell \& O’Reilly, 1990; organizational goal

769 congruence: Vancouver \& Schmitt, 1991; work value congruence: Meglino, Ravlin, \& Adkins,

770 1992; person-organization fit: Cable \& Judge, 1996; O’Reilly et al., 1991; value-resource fit:

771 Roberts \& Robins, 2004; need-press fit: Harms et al., 2006). Usually, the person variables

772 represent different forms of enduring $\mathbb{P}$ (e.g., values, needs, goals, abilities), and the environment

773 variables different forms of enduring e (e.g., presses, affordances, demands, or supplies from

774 another person or group, team, job, vocation, organization, neighborhood, city, region, or

775 culture). In essence, most person-environment fit theories and models thus consider the person

776 variables to be traits and the environment variables to be a rather enduring socio-ecological

777 niches. However, as Table 6 alerts us, there could be also several other forms of person-

778 environment fit that rely on different interpretations of the person and environment variables.

779 Details on profile analyses can be found in Box 3. While person-environment fit has been

780 studied mainly in industrial/organizational psychology, there is also already some research from

781 social and personality psychology. This section serves to just briefly highlight findings especially

782 (though not exclusively) from the latter strings of research. 

- Figure $5-$

In terms of the stability of person-environment fit (path m, Figure 2), previous studies have demonstrated that there is almost no or only little mean-level change (with somewhat increasing means) and substantial rank-order stability ( $r$ s between .30 s to $.60 \mathrm{~s}$ ) across years (Chatman, 1991; Harms et al., 2006; Richards, 1993; Roberts \& Robins, 2004). These studies, however, all concern fit between trait and niche variables in academic or organizational contexts, and thus the generalizability of their findings to other forms of fit (Table 6) and different context domains remains open. More recently, Rauthmann and Sherman (in prep.) examined the levels, stabilities, correlates, and cross-method generalizability (across self- and other-ratings) of patterned person-environment correlations (which they interpreted as "fit"). To do so, the computed $q$-correlations across 31 items for $\dddot{\mathrm{p}} \dddot{\mathrm{E}}$ (asymmetrical) and $\dddot{\mathrm{P}} \mathrm{E}$ fits (symmetrical) from four waves of data, each days to weeks apart. Several findings stand out. First, the normative person and environment variable profiles were substantially correlated $(q \mathrm{~s}=.64-.77)$, suggesting relatively high normative person-environment fit. Indeed, overall fits were, on average, higher, more reliable, more strongly intercorrelated, more temporally rank-order stable, and more strongly correlated in nomologically sensible ways with self-reported intrapersonal adjustment variables (depression, happiness, ego-control, well-being) than distinctive fits. This means that, at least in this study, the links between patterned person-environment fit and adjustment could be traced back to normativity. Second, in almost all cases, there was sizable variance in individual $q$ correlations, thus suggesting the presence of inter-individual differences in patterned personenvironment fits. Third, $\dddot{\mathrm{PE}}$ fits were somewhat stronger than $\dddot{\mathrm{p}} \dddot{\mathrm{E}}$ fits. Lastly, because different rating sources were used for personality traits (self vs. peer-ratings) and for situation ratings (in situ vs. ex situ), the cross-method generalizability of the patterns of findings could also be 
807

808

809

810

811

812

813

814

815

816

817

818

819

820

821

822

823

824

825

826

827

828

examined. While most patterns held up, there was evidence for common-method biases (e.g., higher intra-source and lower inter-source correlations). Taken together, this study demonstrated that patterned person-environment fit in daily life is at least moderately sizable and that there are inter-individual differences in overall and distinctive forms of such fit that are also somewhat stable and correlated mostly due to normativity with adjustment variables.

\subsection{Person-Environment Fit}

4.3.1 Important Distinctions. The concept of person-environment fit dates back to Plato (Dumont \& Carson, 1995), Parson's (1909) matching model of career decision-making, Lewin's (1936, 1943) field theory, and Murray’s (1938) need-press model (see Edwards, 2008 for a review). Today, it is deeply entrenched in industrial/organizational and work psychology (Caplan, 1987; De Cooman et al., 2019; Edwards, 2008; Edwards, Caplan, \& Harrison, 1998; Edwards \& Shipp, 2007; Harrison, 2007; Kristof, 1996; Kristof-Brown \& Guay, 2011; Pervin, 1968; Schneider, 2001; Schneider, Goldstein, \& Smith, 1995; Spokane, Meir, \& Catalano, 2000), while less often studied in personality and social psychology (cf. Bleidorn et al., 2016; Denissen et al., 2018; Harms, Roberts, \& Winter, 2006; Rauthmann, 2013; Roberts \& Robins, 2004). Schneider (2001) even elevated person-environment fit to one of the most important concepts in entire psychology, noting that "[o]f all the issues in psychology that have fascinated scholars and practitioners alike none has been more pervasive than the one concerning the fit of person and environment" (p. 141).

Despite or maybe even because of its importance, person-environment fit has been operationalized quite differently in the literature, both in conceptual and methodologicalstatistical terms. However, in each case, it may be expressed by, or at least involve, person- 
829 environment interactions or correlations. As previously explained, one can broadly distinguish

830 person-centered, profile-oriented from variable-centered person-environment fit. The person-centered view (see 4.2.2) can model person-environment fit as a $q$-correlation

832 between a profile of person variables and another profile of concordant environment variables.

833 The resulting $q$-correlations are computed for each person which makes it possible to examine the 834 extent of inter-individual differences in profile-level person-environment correlations, their 835 stability, and their nomological correlations with other variables. Further, normative profiles of 836 person and environment variables may also be controlled in such analyses. If such $q$-correlations 837 are referred to as "fit", then this fit concept essentially captures a person-environment correlation 838 at the profile-based person level.

839 The variable-centered view can come in two forms (see 4.1.5 and 4.2.1). First, two 840 variables - a person and an environment variable - may be correlated with each other. At the 841 sample level, the resulting correlation coefficient (often Pearson's $r$ ) is an index of a variable842 based person-environment correlation. However, using Asendorpf's (1990) approach (see Box 2), 843 individual person-environment correlation scores can also be derived and thus inter-individual 844 differences in such correlations correlated across time or with other variables. Second, 845 polynomial regression with response surface analyses may be used to examine to what extent 846 person-environment fit patterns are present when predicting an outcome from the linear main 847 effects of person and environment variables, their respective quadratic (and sometimes even 848 cubic) effects, and their interaction effect (see Box 1). In contrast to the previous conceptions of 849 fit, this fit interpretation does not rely on a correlation between person and environment variables 850 but is constituted by a specified effect pattern (see Humberg et al., 2019 for details on which 851 conditions need to be tested and met). 
Further, it is important to consider how an index of fit is derived and what it means.

853

854

855

856

857

858

859

860

861

862

863

864

865

866

867

868

869

870

871

872

873

874

875

876

According to Edwards et al. (2006), there is an atomistic, molecular, and molar approach. In the atomistic approach, person and environment variables are measured each separately, and then fit indices will be derived (e.g., via profile correlations, individual-level scores of sample correlations, or polynomial regression analyses). However, this also means that there can be many different kinds of person-environment fit (for each of those tabulated in Table 6), depending on which person variables (e.g., morphology, dispositions, abilities, goals, values, virtues, strengths, self-concepts, etc.) and environment variables (e.g., discrete life events, psychological situation characteristics, others' normative values, average neighborhood personality trait levels, organizational atmospheres, etc.) were measured in which fashion (e.g., self-reports vs. informant-reports). For example, Edwards and Cooper (1990) argued that the fit between persons' goals and values with supplies from the environments is a different type or version of fit than the fit between persons' abilities and environments' demands. Thus, for the understanding of what a given atomistic person-environment fit score or effect pattern means, it is crucial to clearly specify and operationalize the involved person and environment variables. In the molecular approach, persons are asked to report on their perceived discrepancies between person- and environment-level entities (e.g., whether their workload is too much for their abilities). In the molar approach, persons are asked to directly report on their own personenvironment fit (e.g., how well they fit to an organization). It has been empirically shown that the atomistic, molecular, and molar approaches are not interchangeable and relate only modestly to each other at best (Edwards et al., 2006). As they do not seem to tap the same concepts, each of them provides a unique window into understanding different aspects of person-environment fit. Apart from these more methodological considerations, there are also functional differences in person-environment fit. For example, a broad distinction can be made between supplementary vs. complementary fit of person and environment variables (Cable \& Edwards, 
2004; Muchinsky \& Monahan, 1987). Supplementary fit can be said to occur when high or low "demands" from an environment meet high or low "supplies" from a person. For example, dutiful situations require high conscientiousness, and intellectual situations require high openmindedness. Thus, person and environment variables supplement each other in these examples. In contrast, complementary fit can be said to occur when high or low "demands" from an environment meet low or high "supplies" from a person. For example, situations where other persons are dominant may require less dominance from an actor (i.e., submissiveness), and those situations where others are submissive more dominance from the actor. Thus, person and environment variables complement each other in this example. The distinction between different types of fit also has implications for the commensurability of person and environment variables (e.g., supplementary fit arguably requires stronger commensurability).

\subsubsection{Processes and Consequences of Person-Environment Fit. If the correlation} between a person variable and a conceptually, contentwise, or practically concordant environment variable, or between profiles of concordant person and environment variables, is interpreted as "fit", then the generic PERM in Figure 2 can accommodate and guide more nuanced models. An example is given in Figure 6 that extends path e of the PERM, showcasing how person-environment fit may predict multiple proximal and distal outcomes that capture the duality of persons' identity (the intrapersonal domain) and reputation (the interpersonal domain; Hogan, 1982).

$$
\text { - Figure } 6-
$$

As explained in more detail below, unique hypotheses concerning the intrapersonal domain are that persons with higher person-environment fit should, on average, be more crosstemporally stable in their trait-relevant enactments, show less personality change, feel more authentic, experience more positive affect in daily life, and report higher life satisfaction. 
901 Concerning the interpersonal domain, they should be easier to judge in their personalities (i.e., be

902 good targets) and be more liked or valued by others in groups. Together, persons with higher

903 person-environment fit could be expected to be well-adjusted and, as such, have more resources

904 at their disposal which would enhance survival and reproduction. Given such crucial importance

905 of person-environment fit, persons may be motivated to attain, and also monitor, specific fits to

906 their environments.

907

To capture all of these complexities, the extended person-environment fit process model

908 presented here is inspired by, and can tie together, diverse literatures (e.g., on identity, self,

909 impression formation, group processes, niche construction, adjustment, adaptation, fitness, etc.).

910 To my knowledge, no study has so far tested all the different effect paths proposed in Figure 6

911 simultaneously, though some isolated effects have already been examined (see cursory survey of

912 the literature below).

913 As can be seen in Figure 6, a person-environment correlation (any form from Table 6),

914 defined here as fit, may first proximally impact trait expressions: specifically, which kinds of

915 affects, behaviors, cognitions, and desires (ABCDs; Wilt \& Revelle, 2015) will be expressed how

916 strongly and how consistently. In other words, person-environment correlations may constrain

917 the repertoire and actualization of personality-relevant dynamics and processes in content, level,

918 and variation. Those trait expressions, dependent upon person-environment correlations, then go

919 on to yield more distal effects for the self (intrapersonal route) and for others (interpersonal route)

920 in the three domains of traitedness, evaluations, and adjustment. In all cases, there can be direct

921 effects (e.g., from trait expressions on adjustment) and more indirect or mediated effects (e.g.,

922 from the person-environment correlation to adjustment, via trait-expressions, traitedness, and

923 evaluations). Further, intra- and interpersonal processes may also depend on and co-influence

924 each other across time. 
Intrapersonally, the ways in which traits are expressed habitually lead persons to develop

926 a sense of their self-predictability (how well they know themselves and can anticipate how they

927 react to certain circumstances). Self-predictability is of course also dependent on several other

928 self- and identity-related variables and processes (Thagard \& Wood, 2015; Morin \& Racy,

929 Chapter 15; Pasupathi \& Adler, Chapter 16), such as (but not limited to) self-consistency, self-

930 verification, self-concept clarity, self-insight, and schematicity of self-concept areas. Self-

931 predictability, in turn, may be associated with own perceptions and feelings of authenticity

932 (feeling in tune with and true to one's "real" self), and both may then lead to different indicators

933 of intrapersonal adjustment (e.g., self-esteem, positive affect, flourishing, mental health, well-

934 being, life-satisfaction, etc.). The degree to which perceived authenticity is tied to intrapersonal

935 adjustment may depend also on persons' perceived valence of the ABCDs as a moderator (e.g.,

936 how personally important or consequential they deem certain ABCDs and/or how valuable they

937 think they are to their socio-cultural reference group).

Interpersonally, the ways in which traits are expressed habitually lead others to develop a

939 sense of targets' predictability (how well they can be "read" and their behaviors, or personalities,

940 predicted). Knowing about targets' predictability may also be related to evaluations of their

941 perceived trueness (how true to themselves they seem and to what extent they are acting "out of

942 character"). Both, in turn, may affect how the target is accepted socially and thus also influence

943 interpersonal adjustment (e.g., liking, status, prestige, leadership emergence, etc.). However, an

944 important moderator will be the socio-cultural valence, normativity, and importance of the

945 involved ABCDs.

946 The process model in Figure 6 rests on several assumptions but also bears novel

947 hypotheses. First, attaining person-environment fit should be a core concern of most persons,

948 though there may of course be inter-individual differences in how strongly persons strive to 
949 pursue fit (motivation) and how much energy and resources they invest (investment). Indeed,

950 Erikson (1968) had already described finding a fitting niche as a task for identity development. It

951 also seems that persons will be more engaged and interested in as well as invest more into

952 environments (e.g., occupations, social roles) that fit with or afford their goals and values (for an

953 application of this principle to women's aspirations for pursuing STEM careers, see Diekman et

954 al., 2017). Schmader and Sedikides (2018) have termed the underlying process of pursuing one's

955 goals in an environment that affords those goals "motivational fluency" and surmised that it

956 fosters perceptions or feelings of self-determination. Thus, persons should be motivated to

957 approach goal-fitting environments and avoid others. Indeed, low person-environment fit may

958 instigate selection effects (Holland \& Nichols, 1964), and even after important life transitions,

959 persons tend to create personality-fitting niches (e.g., Jonkmann et al., 2014; Lüdtke et al., 2011;

960 Zimmermann \& Neyer, 2013).

961 Second, if person-environment fit is normatively so important, then there may be

962 monitoring systems that constantly gauge how well we fit to our environments. Indeed, this is the

963 idea of sociometer theory (Leary et al., 1995; Leary \& Baumeister, 2000) which builds on a

964 fundamental need to belong (Baumeister \& Leary, 1995): persons monitor social cues for

965 acceptance versus rejection, and the resulting experiential outcomes of that monitoring are

966 feelings of self-esteem. Correspondingly, it has been found that stronger forms of psychological

967 fit may decrease uncertainties and increase social validation which then can lead to higher self-

968 esteem (Hardin \& Higgins, 1996; Leary, 1999). Further, persons may “feel right" when person

969 and environment variables are synchronized (Cesario, Grant, \& Higgins, 2004; for similar

970 arguments in the domain of regulatory fit when the manner of activity engagement sustains

971 motivational orientations, see Higgins, 2005). According to Schmader and Sedikides (2018), this

972 may lead persons to actively seek environments that afford "interpersonal fluency" (being oneself 
973 with others). Thus, if persons possess something like a "need to fit" and a corresponding

974 generalized "fitometer" system monitoring the different ways in which they fit to their social and

975 non-social environments, then the system's output may result in feelings of positive affect,

976 authenticity, and self-esteem - all indicators of intrapersonal adjustment. Interesting further

977 questions are then to what extent persons' subjective experiences of fit align with their actual fit

978 (e.g., Edwards et al., 2006) and the extent of inter-individual differences in such fit insights.

979

980

981

982

983

984

985

986

987

988

989

990

991

992

993

994

Third, person-environment fit should be generally tied to both intra- and interpersonal adjustment. ${ }^{14}$ Indeed, person-environment fit itself has been related to and even interpreted as adjustment (French, Rogers, \& Cobb, 1974; Thomas \& Chess, 1977). For example, living in areas matching one's personality has been shown to confer certain benefits and increased lifesatisfaction (Bleidorn et al., 2016; Jokela et al, 2015). Additionally, such adjustment via personenvironment fit may result in greater consistency or stability of personality (Wachs, 1994).

Indeed, Roberts and Robins (2004) found in a longitudinal study that students with higher fit to their university environment showed overall less personality change across time.

Fourth, a consequence of the adjustment of persons with generally high person-

environment fit is that, at least within the domains that the person-environment fit pertains to, this person may be more normative and easier to judge (good target; Funder, 1995, 1999) than persons with less person-environment fit. Indeed, well-adjusted persons seem easier to judge in terms of their personality traits (Human \& Biesanz, 2013; Human et al., 2019), and this should also pertain to those with higher person-environment fits.

Lastly, a downstream effect of person-environment fit processes occurring at subpopulation levels is the non-random assortment of persons to specific environments. This means

\footnotetext{
${ }^{14}$ Per sociometer theory, this seems plausible just at face value because if other persons accept and like a person (interpersonal adjustment), then this person will feel good about themselves (intrapersonal adjustment).
} 
995 that there will be spatial clustering of personality phenotypes (and even genotypes) in certain

996 regions (see, e.g., research on geographical and spatial distributions of personality trait levels:

997 Jokela et al., 2015; Rentfrow, 2013; Rentfrow \& Gosling, in press; Rentfrow \& Jokela, 2017,

998 2020; Rentfrow, Gosling, \& Potter, 2008), which likely confer local fitness optima for “fitting”

999 individuals. Such spatial clustering of personality can, in turn, help explain economic growth

1000 differences between regions (Garretsen et al., 2019). However, geographical personality

1001 distributions and regional variations have also been shown to be the product of societal and

1002 economic changes decades earlier (Obschonka et al., 2018). Historically important and large-

1003 scale changes can also contribute to selective migration, which in turn may be seen as a

1004 macroscopic process of a large amount of persons striving to attain better person-environment fit

1005 (i.e., leaving one environment for a another one; see also 5.2.1.1 A Systematic Account). Indeed,

1006 relations between personality traits and migration patterns have already been documented (e.g.,

1007 Camperio Ciani et al., 2007; Jokela, 2014; Jokela et al., 2008), leading to drifts in both genotypes

1008 and personality phenotypes. Thus, the striving for person-environment fit, coupled with the

1009 affordances from regions, societies, cultures, countries, or nations, can also have implications for

1010 group and social identity (Schmader \& Sedikides, 2018), mobility processes (Oishi, 2017), socio-

1011 cultural processes and person-culture fit (Benet-Martínez, Chapter 10), and population dynamics

1012 (Camperio Ciani et al., 2007; Ciani \& Capiluppi, 2011).

1013 4.4 Person-Environment Transactions

1014 Several theories and models emphasize bidirectional transactions between person and

1015 environment variables across time, development, or age (e.g., Baltes, Lindenberger, \&

1016 Staudinger, 2006; Baltes, Staudinger, \& Lindenberger, 1999; Bandura, 1978; Briley \& Tucker-

1017 Drob, 2014; Caspi, Elder, \& Bem, 1989; Corker \& Donnellan, 2020; Elder \& Shanahan, 2006;

1018 Ford \& Lerner, 1992; Lerner, 1978; Lerner \& Walls, 1999; Magnusson \& Stattin, 2006; Plomin 
1019 et al., 1977; Rowe, 1987; Scarr \& McCartney, 1983; Shiner, 2009). These transactions take the

1020 form that prior person variables predict later environment variables and, vice versa, prior

1021 environment variables predict later person variables. Thus, person-environment transactions

1022 concern the phenomenon that person variables $(\mathbb{P}, \mathbb{P})$ and environment variables $(\mathbb{E}, \mathbb{e})$ may be

1023 related to each other across time, that is, one can temporo-sequentially and/or causally predict the

1024 other. Two issues need to be accounted for here:

1025 1. The timescales of the variables used

1026 2. The patterns of transaction effects across time

1027 4.4.1 Timescales of Variables. As explained earlier, there are rather short-term $(\mathbb{P}, \mathbb{E})$ and 1028 rather long-term variables $(\mathbb{p}, \mathbb{e})$ on the side of the person and the environment, respectively

1029 (Table 2). This distinction corresponds to variables being different in their conceptualizations and 1030 measurement in terms of their proximity/distality, abstractness/specificity, and temporal 1031 resolutions. Thus, when relating person and environment variables to each other across time, 1032 their respective shorter- vs. longer-term timescales need to be taken into account. This is 1033 illustrated in Figure 7. Specifically, if earlier variables predict later variables, four types of 1034 underlying processes can be inferred from the predictions (Figure 7):

1035 1. Longer-term variable predicts longer-term variable (ontogenetic process)

1036 2. Longer-term variable predicts shorter-term variable (manifestation/expression process)

1037 3. Shorter-term variable predicts shorter-term variable (actualgenetic process)

1038 4. Shorter-term variable predicts longer-term variable (accretion/accumulation process) - Figure 7 4.4.1.1 Ontogenetic Processes. A stable, long-term variable can predict another stable,

1041 long-term variable. Such ontogenetic transactions are symmetrical because both person and 1042 environment variables are defined as enduring or only slowly changing. For example, research 
1043 has especially examined how genetic variance (p) becomes associated with environmental

1044 differences (e) across time (Johnson, 2007; Kandler et al., Chapter 7) as well as how personality

1045 traits $(\mathbb{P})$ predict persons' personal, social, and occupational niches $(\mathbb{e})$ and/or vice versa (e.g.,

1046 work niches: Hudson, Roberts, \& Lodi-Smith, 2012; Mortimer \& Lorence, 1979; Roberts, Caspi,

1047 \& Moffitt, 2003; Wille \& de Fruyt, 2014; Woods et al., 2013; relationship niches: Asendorpf \&

1048 Wilpers, 1998; Deventer et al., 2019; Kiff, Lengua, \& Zalewski, 2011; Laceulle \& van Aken,

1049 2018; Mund \& Neyer, 2014; Neyer, Mund, Zimmermann, \& Wrzus, 2014; Mund et al., 2016;

1050 Neyer \& Asendorpf, 2001; Reitz, Motti-Stefanidi, \& Asendorpf, 2016). The processes linking

1051 enduring person and environment variables across time are ontogenetic because they unfold over

1052 longer timescales or even across the lifespan. Usually, personality and developmental

1053 psychologists will be interested in such long-term dynamics and processes (Baltes et al., 1999,

1054 2006; Ford \& Lerner, 1992; Lerner, 1978; Lerner \& Walls, 1999; Wrzus \& Roberts, 2017).

Attending specifically to bidirectional transactions between long-term person and

1056 environment variables (see 4.4.2 Transaction Effect Patterns across Time), it has been argued

1057 that there can be selection and socialization effects. These effects occur because there are "two

1058 mutually supportive life-course dynamics: "social selection," wherein persons select

1059 environments that are correlated with their personality traits, and "social influence," wherein

1060 environmental experiences affect personality functioning" (Caspi et al., 2005, p. 470; Roberts \&

1061 Wood, 2006). Thus, there are two concatenated or interlocked processes occurring, namely that

1062 (1) persons select and/or modulate their environments according to their traits (see Gosling et al.,

10632002 and Gosling, Gaddis, \& Vazire, 2008 for personality-manifestation in personal

1064 environments) and that (2), in turn, these traits are consolidated by the selected environments (via

1065 socialization processes). In this vein, the corresponsive principle of personality development

1066 (e.g., Roberts, 2006; Roberts \& Robins 2004; Roberts \& Wood, 2006) states that traits lead 
1067 persons to select certain environments and make specific experiences in them, and then those 1068 experiences change (not stabilize) those initial traits. According to an overview in Roberts and 1069 Nickel (in press), several studies seem to already have yielded a corresponsive pattern of findings 1070 in different person and environment domains (e.g., a large number of person and life experience 1071 variables in different domains: Lüdtke et al., 2011; Roberts et al., 2003, 2004; Roberts \& Robins, 1072 2004; alcohol-proneness in youth and peer-groups: Scalco, Trucco, Coffman, \& Colder, 2015; 1073 impulsivity and substance use: Kaiser et al., 2016; traits and behavioral problems: Klimstra et al., 1074 2010; neuroticism and stressful or negative life events or stress: Jeronimus et al., 2014; Laceulle 1075 et al., 2015; Luo et al., 2017; temperament and relational aggression: Atherton et al., 2017;

1076 effortful control and behavioral problems at school: Atherton et al., 2019; traits and goals:

1077 Bleidorn et al., 2010; Big Five traits and job characteristics: Denissen et al., 2014). However, 1078 there is also evidence for non-corresponsive patterns (e.g., Wood \& Harms, 2016). Indeed, not all 1079 ontogenetic transactions need to follow a corresponsive pattern, and corresponsive effects may 1080 not operate for longer periods of time (Roberts \& Nickel, in press) as there must be some form of 1081 counter-regulative mechanism to prevent ever-more change occurring in the system ad infinitum. There are at least three issues to keep in mind when examining the literature on 1083 corresponsive effects. First, several studies did not actually measure environmental variables, but 1084 focused more on persons' experiences (which are thought of as the extensions of engaging with 1085 certain environments). Thus, indeed corresponsive person $\rightarrow$ person transactions have been studied more often than actual person $\rightarrow$ environment transactions. Second, the corresponsive 1087 principle is about change, but it can easily be confused as being about continuity or stabilization. 1088 Thus, the niche-picking principle has been introduced (Nye \& Roberts, 2019; Roberts \& Damian, 1089 2017; Roberts \& Nickel, 2017, in press) which maintains that persons with certain trait levels 1090 enter or create certain niches that, in turn, serve to deepen or stabilize (not change) their initial 
1091 traits. Indeed, this idea has already been brought forward by Bowers (1973) who noted that

1092 "persons foster consistent social environments which then reciprocate by fostering behavioral 1093 consistency" (p. 329). So far there is only indirect evidence for a niche-picking pattern (better

1094 person-environment fit results in less change: Harms et al., 2006; Roberts \& Robins, 2004) as this 1095 principle has not yet been directly tested. Lastly, many studies could not properly disentangle 1096 within-person from between-person effects (and artifacts). It thus remains an open question to 1097 what extent findings will be replicable when employing different designs and data-analytical 1098 procedures.

4.4.1.2 Manifestive/Expressive Processes. Second, a stable, long-term variable can

1100 predict a more instable, short-term variable. Such manifestive transactions are asymmetrical 1101 because the person and environment variables are defined at different timescales. For example, 1102 personality traits $(\mathbb{P})$ may predict the characteristics of situations $(\mathbb{E})$ persons find themselves in 1103 (Rauthmann et al., 2015c) or more enduring characteristics of persons' daily situations (e) may 1104 predict personality states $(\mathbb{P})$. In such instances, one variable can be said to manifest in or be 1105 expressed by another variable at a smaller, more fine-grained timescale. The concrete underlying 1106 processes unfolding in such manifestive or expressive transactions can be manifold and depend 1107 on the variables being studied.

4.4.1.3 Actualgenetic Processes. Third, a more instable, short-term variable can predict another instable, short-term variable. Such actualgenetic transactions are symmetrical because 1110 both the person and environment variables are defined as fast-changing or momentary variables. 1111 For example, research has examined how prior self-reported personality states $(\mathbb{P})$ predict later 1112 perceived situation characteristics $(\mathbb{E})$ and vice versa in experience sampling data (Rauthmann et 1113 al., 2016). Further, a plethora of experimental and social-psychological research has linked 1114 momentary cues or characteristics $(\mathbb{E})$ of situations to different momentary thoughts, feelings, 
1115 motivations, and behaviors $(\mathbb{P})$ of persons (e.g., Reis, 2008; Richard, Bond, \& Stokes-Zoota,

1116 2003). The processes linking short-term person and environment variables across time are

1117 actualgenetic because they reflect ongoing dynamics and how the transaction unfolds in short-

1118 term timespans. While social psychology has been traditionally interested in such environment-

1119 to-person processes, personality psychology has recently become more invested in understanding

1120 more fine-grained timespans of personality dynamics, processes, of functioning (e.g., Baumert et

1121 al., 2017; Blum et al., Chapter 2; Dermody et al., 2017; Fleeson, 2001, 2012; Hopwood, 2018 ;

1122 Wright \& Hopwood, 2016; Zimmermann et al., 2019), often employing idiographic or within-

1123 person perspectives linking short-term variables to each other (e.g., Beck \& Jackson, Chapter 4;

1124 Fleeson, 2017; Vazire \& Sherman, 2017).

1125 4.4.1.4 Accumulative/Accretive Processes. Lastly, a more instable, short-term variable

1126 can predict a stable, long-term variable. Such accretive transactions are asymmetrical because the

1127 person and environment variables are defined at different timescales. For example, this would be

1128 the case when situation variables $(\mathbb{E})$ predicted personality traits $(\mathbb{P})$ or when personality states

$1129(\mathbb{P})$ predicted niche variables $(\mathbb{e})$. These kinds of processes can, for example, be found in the

1130 literature on the effect of life events or trauma on later development. However, in most of

1131 personality psychology, these processes have received comparatively less attention than the other

1132 process forms outlined before. One reason (besides measurement issues) may lie in the timescale

1133 asymmetry: most scholars would not believe that a single momentary person or environment

1134 variable, respectively, could impact a more stable environment or person variable, respectively.

1135 However, there are at least two instances where this may be the case.

1136 First, some single shorter-term life events $(\mathbb{E})$ can instill or represent "time-discrete

1137 transitions that mark the beginning or the end of a specific status" (Luhmann et al., 2012, p. 594)

1138 and afford changes in demands, roles, status, or relationships. These could be so important (e.g., 
1139 first long-term romantic relationship, first job), consequential (e.g., marriage, divorce, new job,

1140 parenthood), or even traumatic (e.g., losing one's job, death of a partner, being abused, having a

1141 bad accident) that they engender changes in long-term person variables (Bleidorn \& Denissen, in

1142 press; in some dire cases, even growth: Baltes et al., 1999; Jayawickreme \& Blackie, 2014, 2016;

1143 cf. Mangelsdorf, Eid, \& Luhmann, 2019). For example, Bleidorn, Hopwood, and Lucas (2018)

1144 concluded in their comprehensive review of the extant empirical literature that there is "some

1145 evidence that life events can lead to changes in Big Five personality traits and that different life

1146 events may be differently related to specific trait domains", though they cautioned at the same

1147 time that "a more general conclusion emerging from [the] review is that the evidence for the

1148 nature, shape, and timing of personality trait change in response to life events is still at a

1149 preliminary stage” (p. 83). Most studies use a discrete or nominal account of a life event (i.e.,

1150 whether it occurred or not), while failing to account for the potentially moderating roles of

1151 persons' perceptions and anticipations of life events (e.g., their valence, importance,

1152 predictability, controllability, extraordinariness, etc.; see Luhmann et al., 2020 for a taxonomy

1153 and measure) and the processes unfolding before, during, and after the event that may lead to

1154 stabilization or destabilization of personality traits (Roberts \& Nickel, in press). Further, some

1155 short-term person variables $(\mathbb{P})$ may also be hugely consequential for one's later niche $(\mathbb{e})$, for

1156 example, when single acts of delinquency or crime (e.g., murder) lead to imprisonment, or when

1157 a heroic act or a very creative idea may change someone's social status.

1158 Second, several momentary variables may accumulate over time in their effects on a more

1159 enduring variable. In this vein, the repeated exposure to certain situations (thus, E becoming $\overline{\mathrm{E}}$ )

1160 may be accompanied by, or instill, changes in long-term personality traits (p) across time (e.g.,

1161 repeated instances of daily hassles, partner conflict, or bullying could engender more neurotic or

1162 hostile trait levels; repeated environmental exposures manifest in epigenetic biomarkers). Further,

1163 recent research has examined how interventions, and especially forms of psychotherapy (Roberts 
1164 et al., 2017), may instill personality change (for a review, see Hudson, Chapter 50). Such

1165 interventions constitute repeated exposures to certain situations $(\overline{\mathrm{E}})$, which, for example, is

1166 exactly the case in exposure therapy (e.g., Abramowitz, Deacon, \& Whiteside, 2019). Conversely,

1167 the repeated enactment of certain person variables (thus, $\mathrm{P}$ becoming $\overline{\mathrm{P}}$ ) could have profound

1168 impacts on the niches persons craft for themselves in their daily lives and across the lifespan.

1169 4.4.1.5 Summary. Taken together, when examining transactions between person and

1170 environment variables across time, it is important to specify for each variable at which timescale

1171 it resides. Depending on the timescales of the transacting variables, different underlying

1172 processes or types of transactions are examined (i.e., ontogenetic, manifestive/expressive,

1173 actualgenetic, accretive/accumulative). Notably, even when examining only shorter-term or

1174 longer-term variables, respectively, there may still be differences in proximity/distality,

1175 abstractness/specificity, and temporal resolutions among them (e.g., within shorter-term

1176 variables: unfolding across seconds vs. minutes vs. days; within longer-term variables: e.g.,

1177 culture is at a different level of abstraction than one's neighborhood, and both are different from

1178 one's usual social networks). Further, "shorter-term" vs. "longer-term" should be seen on a

1179 continuum rather than as discrete categories. Keeping these issues in mind, the next section

1180 (4.4.2) concerns the patterns of effects of persons on environments and environments on persons.

1181 Each of those effects may be ontogenetic, manifestive/expressive, actualgenetic, or

1182 accretive/accumulative depending on how the variables have been conceptualized and measured.

1183 4.4.2 Transaction Effect Patterns across Time. Given a person and an environment

1184 variable each measured at two time-points, there are two stability paths (of the person and of the

1185 environment variable; see paths $\mathrm{j}$ and $\mathrm{k}$ in Figure 2) and two cross-lagged paths (from the person

1186 to the environment variable, and from the environment to the person variable; see paths $\mathrm{n}$ and o

1187 in Figure 2) in the PERM. Depending on which of these four effects are "present" (i.e., 
1188 statistically significant, sizable, or meaningful) across time, 16 different kinds of transaction

1189 effect patterns can be identified. These patterns are summarized in Table 7 and depicted in Figure

1190 8. Additional patterns could be identified when also considering the cross-sectional presence or

1191 absence of person-environment correlations; however, for simplicity, these were not included in

1192 Figure 8. Broadly, four different kinds of transaction effect patterns can be distinguished:

1193 1. Non-transactions

1194 2. Bidirectional transactions (persons $\rightarrow$ environments, environments $\rightarrow$ persons)

1195 3. Monodirectional transactions of persons $\rightarrow$ environments

1196 4. Monodirectional transactions of environments $\rightarrow$ persons

1197

1198

1199

1200

1201

1202

1203

1204

1205

1206

1207

1208

1209

1210

1211
- Table $7-$

- Figure 8 -

First, there are four non-transaction effect patterns. These patterns have been termed "non-transactional" because there are no effects from person to environment variables or from environment to person variables. One pattern (Zero Transaction) does not contain any effects at all, and the other patterns (Person Stability, Environment Stability, and Bi-Stability) only contain certain stability paths. Although such patterns may exist (e.g., some evidence for a Bi-Stability pattern in experience sampling data with P and E: Rauthmann et al., 2016), they do not capture genuine transactions between persons and environments.

Second, there are four bidirectional effect patterns which capture "transactions" how they are traditionally conceptualized: as mutual, reciprocal, or bidirectional relations between person and environment variables across time. For all of these effect patterns, the two cross-lagged effect paths are present, and they are distinguished only regarding the presence of stability effects (both present: Full; none present: Reset; only person-stability: Person-tilted; only environmentstability: Environment-tilted). It is especially noteworthy that these bidirectional transactions will 
1212 lead to (higher) person-environment correlations across time (see Fraley \& Roberts, 2005 for a

1213 simulation study), though not infinitely so as there are natural and measurement boundaries as

1214 well as likely counter-regulative mechanisms acting against an ever-increasing correlation (i.e., it

1215 will stagnate at some point, reaching its asymptotic maximum, or even reverse). Moreover,

1216 looking to transactions in person-culture processes (see Benet-Martínez, Chapter 10), Markus and

1217 Kitayama (2010) stated that "[i]n a cycle of mutual constitution, people are culturally shaped

1218 shapers of their environment" (p. 421). Even more macroscopically, gene-culture co-evolution

1219 may occur as a result of the chronic unfolding of, and bidirectional effects between, genes and

1220 culture in populations (for a review, see Laland, Odling-Smee, \& Myles, 2010).

1221 Lastly, there are eight monodirectional transaction patterns where effects flow only into

1222 one direction: four patterns concern person variables predicting later environment variables (but

1223 not vice versa), and four other patterns environment variables predicting later person variables

1224 (but not vice versa). While these patterns are also termed transactions here, the temporal and/or

1225 causal impacts are one-sided and not reciprocal.

1226 All transaction effects in Table 7 and Figure 8 could potentially be ontogenetic,

1227 manifestive/expressive, actualgenetic, or accretive/accumulative depending on how the respective

1228 person and environment variables have been operationalized and which research designs have

1229 been used. However, some effect patterns may be more likely or more pronounced with certain

1230 variables than others. For example, Denissen et al. (2014) have found different transaction

1231 patterns (e.g., bidirectional and monodirectional ones) depending on whether participants were

1232 job beginners, job stayers, and job changers as well as which Big Five trait was examined (see

1233 corresponding transaction patterns in their Figure 1, p. 1936). Further, with more than two

1234 measurement occasions, different effect patterns can be concatenated and yield more complex

1235 ones. In addition, patterns may develop, evolve, or change across time, becoming weaker or 
1236 stronger (e.g., when more stabilization or destabilization occurs). This makes it possible to

1237 identify cascades and vicious cycles (which still do not continue indefinitely, though).

1238 4.4.2.1 Findings. This section necessarily contains only a few selected findings from the

1239 vast literatures that have examined any form of lagged or even cross-lagged effects of person and

1240 environment variables. Details on the analysis of reciprocal or cross-lagged person-environment

1241 transactions can be found in Box 4. Importantly, many studies and meta-analyses have examined

1242 cross-lagged effects, but most did not situate their findings explicitly within a person-

1243 environment relations framework. Additionally, there are several studies that have quantified

1244 only one path (e.g., $\mathrm{n}$ in the PERM: prior traits p predict later situation characteristics E; Mehl,

1245 Gosling, \& Pennebaker, 2006; Rauthmann et al., 2015c; Wrzus, Wagner, \& Riediger, 2016) and

1246 thus established one direction but not the other. Those will not be referenced here because they

1247 cannot be used to glean full information on the different transaction patterns in Table 7 and

1248 Figure 8 . The following cursory summary intends to provide an impression of the effect sizes and

1249 transaction effect patterns found in the domains of (1) life events, (2) work, (3) relationships, and

1250 (5) daily life.

4.4.2.1.1 Life Events. In terms of various live events in general (which often are also

1253 work- and relationship-related, but not exclusively so), there may sometimes be full bidirectional

1254 transactions between person and environment variables (Bleidorn et al., 2018; Bleidorn \&

1255 Denissen, in press; Roberts, \& Nickel, in press). However, there generally seems to be a trend

1256 pointing towards stronger paths from persons to environments than vice versa. For example,

1257 Bleidorn et al. (2018) found only rare instances in which life events instilled lasting personality

1258 changes as a consequence. Further, Kandler et al. (2012) found in a longitudinal twin study that

1259 directionality primarily flowed from personality traits to life events, and that relations between 
1260 traits and life events were mostly genetically mediated. Nonetheless, once controlling for genetic 1261 associations, they found small environmental mediations for effects of life events on traits.

1262 Some contextual personality (development) theories, such as Social Investment Theory

1263 (SIT; Roberts, Wood, \& Lodi-Smith, 2005), claim that "experiences in (...) social roles, as well

1264 as expectations for role-appropriate behavior, contribute to changes in personality traits" (Lodi-

1265 Smith \& Roberts, 2007, p. 81). In contrast, more endogenous or biologically oriented personality

1266 theories, such as Five-Factor Theory (FFT; McCrae \& Costa, 2008), deny the influence of

1267 environmental variables on trait variables as "traits are immune to the effects of the psychological

1268 environment" (McCrae, 2019, p. 64). The only exception to this principle is that environmental

1269 influences (e.g., nutrition, physical trauma, physical activity, psychopharmacological treatment)

1270 may impact biological structures and functions which can, in turn, cause downstream effects in

1271 basic traits (McCrae, 2019; see also DeYoung, 2015). There is also evidence supporting this view

1272 as true long-term personality change after life events has been rarely demonstrated (Costa et al.,

1273 2000; Costa, McCrae, \& Löckenhoff, 2019; McCrae et al., Chapter 23). Together, SIT posits

1274 profound socialization effects, while FFT does not; in contrast, FFT posits strong selection

1275 effects. The disagreement between contextual and endogenous theories lies thus in whether basic

1276 traits, such as the Big Five, can change enough and lastingly with the objective exposure to

1277 and/or subjective experience of life events; however, both agree that characteristic adaptations

1278 (such as goals) may be impacted by life events.

1279 Despite scarce evidence for lasting personality change in the face of life events, there are

1280 some studies that suggest the power of life events to shape personality. For example, traveling

1281 abroad - and thus being exposed for a longer time to a new culture and many new experiences -

1282 may have effects on personality development (Lüdtke et al., 2011; Zimmermann \& Neyer, 2013).

1283 Further, Denissen et al. (2019) also found in a large, nationally representative, and prospective 
1284 longitudinal study some small socialization effects (i.e., life events $\rightarrow$ traits), but two thirds of all

1285 effects were still identified as selection effects (traits $\rightarrow$ life events). Further, socialization effects

1286 can be either anticipatory (some life events can be anticipated) or reactionary (some cannot be

1287 anticipated and just have to be experienced and dealt with).

1288 4.4.2.1.2 Work. In terms of work (e.g., employment/unemployment, vocation, salary,

1289 etc.), several studies point towards full bidirectional transactions. For example, Roberts et al.

1290 (2003) found a corresponsive pattern in that prior personality traits at age 18 predicted later

1291 (subjective and objective) work experiences at age 26, while the work experiences were related to

1292 personality changes from age 18 to 26. In a longitudinal design using job beginners, changers,

1293 and stayers, Denissen et al. (2019) could tease apart selection from socialization. Specifically,

1294 there were selection effects for job beginners and changers (i.e., prior trait levels predicted later

1295 job demands) and also socialization effects for job stayers (i.e., prior job demands predicted later

1296 trait levels). Ford et al. (2014) conducted a meta-analysis on studies using multiple measurements

1297 of job-related stressors and psychological or physical strains. They found small cross-lagged

1298 effects in both directions (stressors $\rightarrow$ psychological/physical strains: .03 and .05;

1299 psychological/physical strains $\rightarrow$ stressors: .01 and .04), which - if deemed practically important

1300 - may be interpreted in support of bidirectional transactions.

1301 4.4.2.1.3 Relationships. In terms of social, romantic, and familial relationships (e.g.,

1302 entering a certain type of relationship; relationship quality, quantity, conflict, etc.; social support

1303 from others), there may also be full bidirectional transactions, but most currently available

1304 findings point towards effects being more frequent and stronger for person(ality) $\rightarrow$ relationships

1305 paths than vice versa (e.g., Asendorpf \& van Aken, 2003; Asendorpf \& Wilpers, 1998; Deventer

1306 et al., 2019; Mund \& Neyer, 2014; Neyer \& Asendorpf, 2001; Neyer \& Lehnart, 2007). Indeed, 
$130781 \%$ of significant cross-lagged paths concerned effects from traits to relationship variables in

1308 Deventer et al.'s (2019) study, and about two thirds were related to trait facets (rather than trait

1309 domains). Nonetheless, there are also studies that demonstrate some sizable or important

1310 relationships $\rightarrow$ person(ality) effects (e.g., Sturaro, Denissen, \& van Aken, 2008), especially

1311 when examining relations at the facet level of traits and using appropriate designs and modeling

1312 procedures (Mund \& Neyer, 2014). Wrzus and Neyer (2016) provided a lifespan perspective in

1313 reviewing empirical studies on the co-development of traits and relationship variables across the

1314 lifespan. They also reported that selection effects were somewhat stronger than socialization

1315 effects. However, there were important developmental patterns for selection effects of personality

1316 on friendships (small to moderate in adolescence, moderate to large in young adulthood, small or

1317 negligible in middle and late adulthood) and on familial relationships (small in adolescence and

1318 young adulthood, moderate in middle and late adulthood). Further, socialization effects also

1319 showed age-graded changes (moderate during adolescence, small to negligible from young

1320 adulthood onwards). These findings suggest that transaction patterns need to be considered in

1321 conjunction with life phases in persons' development (Wrzus et al., 2016).

4.4.2.1.4 Daily Life. The preceding studies reviewed for life-events, work, and

1323 relationship domains concerned mostly long-term variables and thus ontogenetic processes. In an

1324 experience sampling study looking at actualgenetic transactions within the timeframe of hours,

1325 Rauthmann et al. (2016) predicted later perceived situation characteristics from prior personality

1326 states, and later personality states from prior situation characteristics. They could compare

1327 altogether 80 effects (i.e., all possible combinations between the sampled person and environment

1328 variables), of which 23 were deemed a priori as conceptually or prima facie plausible and 57 as

1329 not plausible. Across all 80 combinations, absolute situation characteristic $\rightarrow$ personality state 
1330 effects averaged to $0.03^{13}(S D=0.02)$, and absolute effects were higher for plausible $(M=0.05$, $1331 S D=0.02)$ versus non-plausible combinations $(M=0.03, S D=0.02)$. Similarly, across all 80

1332 combinations, absolute state $\rightarrow$ situation characteristic effects averaged to $0.03(S D=0.02)$, and

1333 absolute effects were higher for plausible $(M=0.04, S D=0.02)$ versus non-plausible

1334 combinations $(M=0.02, S D=0.02)$. Thus, while the cross-lagged paths were comparable in

1335 magnitudes, they were relatively small in all instances. This pattern of findings would speak more

1336 for a Bi-Stability model (4 in Figure 8) where mainly the stabilities of the person and

1337 environment variables is present across time (in Rauthmann et al., 2016; person variables with $M$ $1338=0.20, S D=0.03$; situation characteristic variables with $M=0.22, S D=0.07$ ). 4.4.2.2 Summary. For long-term variables and ontogenetic timescales, the following 1340 tendencies seem to crystallize across literatures. First, the stability of trait variables tends to be

1341 somewhat higher (path j) than that of niche variables (path k). Second, cross-lagged effects from

1342 person to environment variables (path $\mathrm{n}$ ) tend to be stronger than from environment to person 1343 variables (path o). Third, combining both prior insights, full bidirectional transaction patterns (5 1344 in Figure 8) are often hypothesized to occur, but the empirical evidence is currently somewhat 1345 stronger for the person-tilted monodirectional or even full person transaction pattern (9 and 10 in 1346 Figure 8, respectively). For short-term variables and actualgenetic timescales, transaction effects 1347 are often small. Nonetheless, these must exist (e.g., when cues in the environment are processed 1348 by a perceiver and elicit affective reactions), even if they are difficult to detect or disentangle. In 1349 general, however, these summary observations are all just broad-stroke descriptions across 1350 various effects in the literature, and of course other patterns have also been found.

1351 There are several explanations for the apparent "ontogenetic power" of personality to 1352 shape niches more so than niches shape personality: (1) stability of variables, (2) breadth of 1353 variables, (3) transition points, (4) developmental periods, and (5) research methodologies. First, 
1354 traits are believed to be generally relatively stable variables, both in terms of mean-level

1355 (Roberts, Walton, \& Viechtbauer, 2006) and rank-order stability (Roberts \& DelVechhio, 2000).

1356 In contrast, environments or niches may be less stable. Indeed, there is considerable mean-level 1357 change of daily situation characteristics across the life-span (Brown \& Rauthmann, 2016), and 1358 the studies examining personality-relationship transactions have also found relationship variables 1359 to be often less stable than the trait variables (e.g., Deventer et al., 2019; Mund \& Neyer, 2014).

1360 More stable variables could perhaps be in a better position to impact less stable variables more 1361 strongly (than vice versa), which may explain why traits tend to predict relationship variables 1362 more often and more strongly than vice versa (Neyer et al., 2014; Wrzus \& Neyer, 2016). More 1363 stable variables are by definition less malleable than more fluctuating variables, and their effects 1364 could be less time-dependent - thus ensuring stronger predictive power.

Second, environment variables often reside at a different granularity level than broad-

1366 stroke traits (e.g., the Big Five domains). It might be too difficult for broad and abstract variables 1367 to predict narrow and specific variables (bandwidth-fidelity dilemma or trade-off; Cronbach \& 1368 Gleser, 1957; Hogan \& Roberts, 1996), and this asymmetry may explain the lack of evidence for 1369 socialization effects (Mund \& Neyer, Chapter 9). Further, domain-level traits are indeed less 1370 related to relationship variables than facet-level traits, and some relationship $\rightarrow$ personality 1371 effects can only be uncovered at the facet-level of traits (Mund \& Neyer, 2014). Thus, the level 1372 of analysis and breadth of variables is important to consider.

1373 Third, whether normative or non-normative life transition points (e.g., entering or exiting 1374 a relationship, entering the first or a new job, etc.) are included in the analyses may make a 1375 difference. For example, Caspi and Moffit $(1991,1993)$ have argued that strong or disruptive life 1376 events may - paradoxically - accentuate, reinforce, or even stabilize individual differences 1377 instead of changing them. This may, however, only hold for historical and large-scale events 
1378 (e.g., pandemics, wars, recessions) that affect an entire population or for age-graded events that

1379 rather uniformly affect a certain age slice of the population (e.g., schooling, retirement). If there

1380 are additionally barely any inter-individual differences in intra-individual stabilities and changes,

1381 then environment effects on trait levels or changes will be hard to detect. Further, transitive

1382 events may still offer the opportunity to create niches that are congruent with one's existing

1383 personality traits (see 4.3.2. Processes and Consequences of Person-Environment Fit and 5.

1384 Person-Environment Calibrations and Navigation Mechanisms). Together, studying person-

1385 environment or relationship transactions in times of transitions may actually make it harder to

1386 detect bidirectional transactions because individual differences may stabilize (Neyer et al., 2014).

1387 It is important to keep in mind that persons are often (though not always) neither randomly nor

1388 passively exposed to life events. As such, personality influences on the anticipation, occurrence,

1389 unfolding, and consequences of life events are to be expected.

1390 Fourth, an even more differentiated picture on person-environment transactions can be

1391 obtained from a developmental lifespan perspective. Opportunities, possibilities, and constraints

1392 are age-graded (Baltes, 1987, 1997), and thus person-environment transactions should show

1393 differences across the lifespan (Lang, Reschke, \& Neyer, 2006; Wrzus et al., 2016).

Lastly, on their treatise on personality-relationship transactions, Mund and Neyer

1395 (Chapter 9) raised attention to methodological issues that also take into account the preceding

1396 points. Such issues concern the research designs (e.g., using mixed environments, having

1397 transitions or not, obtaining dyadic data, having several measurement bursts, ensuring age

1398 homogeneity vs. heterogeneity of participants and attending to the age range, sampling facet-

1399 level data, etc.) and the data-analytical techniques employed (e.g., cross-lagged panel models and

1400 newer inventions; Mund \& Nestler, 2019). It should be noted that these issues do not just pertain

1401 to personality-relationship transactions, but also to person-environment transactions in general. 


\section{Person-Environment Calibrations and Navigation Mechanisms}

Persons constantly navigate environments, and those environments provide a rich smorgasbord of opportunities and obstacles. Hence, persons and environments can exert influences upon each other so that each of them may change in corresponding ways, and they can also become systematically correlated across time. These processes are summarily referred to here as calibrations, and they are different from simple stability or change in each of the person and environment variables. The time-lagged paths in Figure 2 ( $\mathrm{j}$ and $\mathrm{k}$, and $\mathrm{n}$ and $\mathrm{o}$ ) contain all types of stability and change, but calibration more specifically refers only to change in response to something, often though not always also for a functional purpose (especially on the side of persons). Such calibrations may be achieved by different person and/or environment navigation mechanisms (NMs) and they may result in person-environment correlations via those mechanisms (see path $\mathrm{f}^{\prime} / \mathrm{f}^{\prime \prime}$ in Figure 2). Notably, there can be mechanisms in or from the person

$\left(\mathrm{g}^{\prime}\right)$ and in or from the environment ( $\left.\mathrm{g}^{\prime \prime}\right)$. This section outlines different types of calibrations and associated mechanisms which can help foster a more dynamic understanding of persons and environments in conjunction.

\subsection{Calibration Types}

At any given time-point, persons and environments may be inextricably interwoven such that influences come from all directions and go into all directions. Nonetheless, it still makes sense to distinguish between functionally different types of calibrations which each may exert influences upon $\mathbb{P}, \mathbb{P}, \mathbb{E}$, and $e$ variables in isolation as well as on their effects or relations in a concurrent or lagged fashion. There are four different types of calibrations (see Table 8), which can be obtained when crossing two "active/sending" subjects (person vs. environment) with two “passive/reactive/receiving” objects (persons vs. environment): 
1425 1. Person $\rightarrow$ Person calibration

1426 2. Person $\rightarrow$ Environment calibration

1427 3. Environment $\rightarrow$ Person calibration

1428 4. Environment $\rightarrow$ Environment calibration

For each of these four types of calibrations, again two instances can be distinguished.

1431 First, in some way commensurate variables may be calibrated across time (e.g., for Person $\rightarrow$

1432 Person: job satisfaction at different time points). Second, incommensurate variables may also be

1433 calibrated across time (e.g., for Person $\rightarrow$ Person: job satisfaction and family conflicts at different

1434 time points). It is thus always necessary to clearly specify which person and environment

1435 variables are involved in calibrations as well as why and how they are.

1436 In a Person $\rightarrow$ Person calibration (mechanisms drive or moderate path j in Figure 2), a

1437 person changes something about themselves. Such autoplastic person-focused change has already

1438 been documented in the literatures on state fluctuations and their stabilizations (Fleeson, 2012,

1439 2017), life management and selection-optimization-compensation (SOC) strategies (Baltes \&

1440 Baltes, 1990; Freund \& Baltes, 1998, 2000, 2002), self-regulated personality development

1441 (Hennecke et al., 2014), secondary control mechanisms (Heckhausen, 1997), emotion regulation

1442 strategies (Gross, 1998), and developmental regulation (Haase, Heckhausen, \& Wrosch, 2013).

1443 For example, in the pursuit of autoplastic regulation, persons make use of life-management

1444 strategies composed of selection (i.e., setting, specifying, and focusing on goals), optimization

1445 (i.e., acquiring and investing of goal-relevant means), and compensation (i.e., using alternative 1446 means to preserve a function) (Freund \& Baltes, 2002). 

2), a person changes something about the environment. Such alloplastic change has already been

1449 documented in the literature on gene-environment associations (e.g., Johnson, 2007; Kandler et

1450 al., Chapter 7), evolutionarily informed person-environment transactions and adaptive conditional 1451 strategies (Buss, 1987, 1991; Buss \& Greiling, 1999), person-environment fit (e.g,, Kristof-

1452 Brown \& Guay, 2011), primary control mechanisms (Heckhausen, 1997), situational self-control 1453 (Duckworth, Gendler, \& Gross, 2016), complex patterns of personality development (Kandler et 1454 al., 2015; Roberts, 2018; Roberts \& Caspi, 2003), and how persons' lives unfold when they 1455 construct their niches (Tesser, 2002). Specifically, a large body of literature suggests that 1456 personality $(\mathbb{P})$ has the power to shape situations $(\mathbb{E})$ and niches (e) (e.g., Allport, 1961; Bandura, 1457 1978; Diener, Larsen, \& Emmons, 1984; Emmons \& Diener, 1986a,b; Emmons et al., 1985, 1458 1986; Furnham, 1981; Holland, 1966; Scarr, 1996; Snyder, 1981; see Ickes et al., 1997, pp. 1751459184 for a review), generally in motive- or need-congruent ways. Thus, it has been argued that "a 1460 person's selection and creation of environments is one of the most individuating and pervasive 1461 expressions of his or her personality" (Caspi et al., 1989, p. 377). Indeed, Allport (1961, p. 179)

1462 had already maintained that “... most persons do a good deal to create the situation to which they 1463 respond" so that "... the situations we find ourselves in are often the direct product of our 1464 previous (and continuing) personalities." Thus, persons' personalities could be understood by, or 1465 even as a special form of, their "imprints" on their environments (e.g., Gosling et al., 2002, 2008;

1466 Hopwood, 2018; Sullivan, 1953; see also 6.7 A Transactional Perspective on Traits and 1467 Personality).

1468 In an Environment $\rightarrow$ Person calibration (mechanisms drive or moderate path o in Figure 1469 2), an environment changes something about a person. Such "molding" has already been 1470 documented in the literature on effects of experimentally manipulated stimuli on psychological 
1471 functioning and behavior (e.g., Orne, 1962; Reis, 2008); affordances (Chemero, 2001, 2003;

1472 Gibson, 1977; Miller, 2007; Stoffregen, 2000a,b, 2003, 2004); subtle nudges (Hertwig \& Grüne-

1473 Yanoff, 2017; Thaler \& Sunstein, 2008; Sunstein \& Reisch, 2017; e.g., food choices, dieting,

1474 healthy lifestyle: Arno \& Thomas, 2016; policy and markets: Abdukadirov, 2016); processes of 1475 attraction-selection-transformation-manipulation-attrition (ASTMA; Nye \& Roberts, 2019); life

1476 events, work, and relationships on personality development (e.g., Bleidorn et al., 2018; Mund \&

1477 Neyer, Chapter 9; Nye \& Roberts, 2019); situation strength (Cooper \& Withey, 2009; Dalal et al., 1478 2019; Judge \& Zapata, 2015; Keeler et al., 2019; Meyer et al., 2020; Meyer \& Dalal, 2009;

1479 Meyer, Dalal, \& Hermida, 2010; Rauthmannn, 2018c); traumata (e.g., Jayawickreme \& Blackie, 1480 2014, 2016; cf. Mangelsdorf et al., 2019); and psychotherapy and interventions (Hudson, Chapter 1481 50; Roberts et al., 2017). For example, Ickes et al. (1997, p. 166) have stated that "environments 1482 provide opportunities for personal dispositions to be manifested and reinforced" and that the fact 1483 that persons "display ... behaviors in settings they have specifically chosen ensures a substantial 1484 degree of consistency in their behavior." Thus, trait manifestation and behavioral consistency 1485 could also be seen partly as downstream products of environmental variables exerting their 1486 influences (see also Fleeson, 2007; Fleeson \& Law, 2015; Sherman et al., 2015). In an Environment $\rightarrow$ Environment calibration (mechanisms drive or moderate path $\mathrm{k}$ in

1488 Figure 2), an environment changes something about itself. Such an environment-focused change 1489 reflects the inherent dynamics of daily life over which persons sometimes do not have a direct 1490 influence (e.g., waiting for a bus where one cannot influence if or when the bus comes). For 1491 example, they have already been documented in the literature on chance encounters, events, and 1492 developments (e.g., Bandura, 1982; Hirschi, 2010). However, this does not mean that these 1493 processes or influences are random; they simply happen independent of a person and just concern 1494 intra-environment dynamics (e.g., passage of time, natural disasters, social and cultural changes 
1495 and shifts). Moreover, there can be different mechanisms linking different environment variables

1496 to each other (e.g., work and family), such as, for example, spillovers, compensations,

1497 segmentations, resource drains, congruence, and conflicts (see Edwards \& Rothbard, 2000 for a 1498 review).

From this description of the four general types of calibrations, it becomes clear that they

1500 will likely differ in their actual and perceived agency and locus of control for persons. For

1501 example, the following relations seem plausible regarding persons' general levels of agency:

1502 Person $\rightarrow$ Person $\geq$ Person $\rightarrow$ Environment $>$ Environment $\rightarrow$ Person $\geq$

1503 Environment $\rightarrow$ Environment. However, these relations reflect as of yet untested hypotheses.

1504 Interestingly, several empirical studies may suggest stronger person $\rightarrow$ environment than

1505 environment $\rightarrow$ person effects (see $n$ and o paths, respectively, in Figure 2; see also 4.4.2.2

1506 Summary). However, looking at experience sampling data that capture dynamics and processes

1507 unfolding in short-term timespans (e.g., hours), the effects of personality states (P) on perceived

1508 momentary situation characteristics (E) and vice versa are almost identical, though all small

1509 (Rauthmann et al., 2016). Notably, all surveyed studies only pertain to the effect paths found and

1510 did not sample any NMs. However, taken together, the currently available patterns of findings

1511 seem to suggest that - on average and especially across longer periods of time (as opposed to

1512 shorter timespans) - environment $\rightarrow$ person NMs may exert a smaller or less tractable influence

1513 than person $\rightarrow$ environment NMs. If this were the case, then this would be a powerful

1514 demonstration of persons' agency over their lives and their development. Still, no firm

1515 conclusions can be reached to date as the empirical quantification of NMs and their influences

1516 has not progressed so far (see 6.5 Conceptualizing, Measuring, and Studying Calibrations and

1517 Navigation Mechanisms). 


\subsection{Navigation Mechanisms}

The different forms of calibration previously outlined happen and can be achieved via navigation mechanisms (NMs; see Table 8). The following two sections will only focus on alloplastic NMs for person $\rightarrow$ environment and for environment $\rightarrow$ person calibrations, with a stronger emphasis on the former (i.e., how persons get into and out of environments). The autoplastic NMs for person $\rightarrow$ person and for environment $\rightarrow$ environment calibrations will be left aside. Though these are, of course, also important to consider and to understand, they are beyond the scope of this chapter that is more concerned with person-environment relations than with relations within the person or the environment, respectively.

\subsubsection{Navigation Mechanisms for Person $\rightarrow$ Environment Calibrations. Persons have}

been described as constantly striving to create equilibria between their personalities and environments (Cramer et al., 2012). To do so, they may employ - willingly and unwillingly different person $\rightarrow$ environment NMs (Allport, 1937; Buss, 1987; Caspi \& Roberts, 2001; Ickes et al., 1997; Plomin et al., 1977; Rauthmann \& Sherman, 2016; Roberts \& Caspi, 2003; Scarr \& McCartney, 1983; Snyder, 1983; Snyder \& Ickes, 1985). Via the habitual utilization of person $\rightarrow$ environment NMs, persons can try to gradually "optimize" their surroundings according to their personalities (especially, needs, values, and goals), and thus person and environment variables will become correlated or synchronized over time (Fraley \& Roberts, 2005; see also 4.2 Person-Environment Correlations). This fundamental principle has been voiced in multiple, discipline-spanning literatures. Personality, social, and developmental psychological literatures have already detailed how persons structure and regulate their daily lives (e.g., Duckworth, Gendler, \& Gross, 2016; Haase et al., 2013; Ickes et al., 1997; Rauthmann \& Sherman, 2016; Tesser, 2002). Further, industrial/organizational and work psychology has also emphasized how 
1541 persons craft their jobs (e.g., Holland, 1959, 1987; Wrzesniewksi, 2001). Lastly, biologically or

1542 genetically oriented disciplines have also noted persons' capacities to shape their environments

1543 (e.g., niche-picking, niche-building: Scarr \& McCartney, 1983; niche construction: Laland \&

1544 Brown, 2006; genotype-specific habitat selection: Hedrick, 1990; experience-producing drives:

1545 Bouchard, Lykken, Tellegen, \& McGue, 1996). Still, niche construction seems to have been a 1546 neglected process in evolutionary biology (Laland \& Sterelny, 2006; Odling-Smee, Laland, \&

1547 Feld, 2013). However, recent efforts have been made to develop a niche construction theory (for 1548 reviews, see Laland, Matthews, and Feldman, 2016 and Scott-Phillips et al., 2014), focused on 1549 "the process whereby organisms, through their metabolism, their activities, and their choices, 1550 modify their own and/or each other's niches" (Odling-Smee et al. 2003, p. 419). Indeed, niche 1551 construction seems to be - besides natural selection - a key driver of evolution as " $\mathrm{t}] \mathrm{he}$ 1552 properties of environments cause (some of) the properties of organisms through the action of 1553 natural selection, but equally the properties of organisms cause (some of) the properties of 1554 selective environments through niche construction" (Laland, 2004, p. 316). Taken together, in the 1555 long haul, person $\rightarrow$ environment NMs may thus stand in the service of (active) developmental 1556 regulation (Baltes, 1997; Caspi et al., 2005; Haase et al., 2013; Roberts \& Caspi, 2003), 1557 ontogenetic gene-environment correlations (Plomin et al., 1977, 2008), and even cultural and 1558 evolutionary processes (Arslan \& Penke, 2015; Day, Laland, \& Odling-Smee, 2003; Kendal, 1559 Tehrani, \& Odling-Smee, 2011; Laland, 2004; Laland, Odling-Smee, \& Feldman, 1996, 1999;

1560 Nettle \& Penke, 2010; Penke, 2010; Penke, Denissen, \& Miller, 2007a,b; Penke \& Jokela, 2016).

1561 For example, concepts from evolutionary psychology such as frequency-, density-, and 1562 competition-dependent selection imply that different genes, behavioral syndromes, and 
1563 environmental resources are distributed differently and can have profound adjustment, fitness,

1564 and phylogenetic effects (Penke, 2010). ${ }^{15}$

5.2.1.1 A Systematic Account. Table 9 summarizes general person $\rightarrow$ environment NM

1566 types from different literatures (see Rauthmann \& Sherman, 2016 and Rauthmann, 2018c who

1567 referred to these as "situation management strategies"). The person $\rightarrow$ environment NMs in Table

15689 may differ with respect to different properties (e.g., intentionality, effort, control, extent of

1569 actual change induced, etc.), but these remain to be empirically tested (see later also 6.5

1570 Conceptualizing, Measuring, and Studying Calibrations and Navigation Mechanisms for more

1571 open questions). The different person $\rightarrow$ environment NM types can also be brought into a

1572 meaningful system, as depicted in Figure 9. Notably, the person $\rightarrow$ environment NMs may operate

1573 and unfold at both actualgenetic and ontogenetic timescales, depending also on the timescales of

1574 the person and environment variables involved in the calibration. Further, they should not be

1575 viewed as mutually exclusive, but some can be combined, mixed, or used simultaneously. Lastly,

1576 each person $\rightarrow$ environment NM type may have its advantages and disadvantages, and so their

1577 relative (dys-)functionalities may vary across and within persons and environments.

1578

1579

1580

1581
- Table 9 -

- Figure 9 -

At a very basic level from an agency perspective of a person, a person may have the opportunity to influence something about her-/himself (autoplastic mechanisms), the current

\footnotetext{
15 The PERM currently concerns only more actualgenetic and ontogenetic processes explicitly. While phylogenetic processes (e.g., transformation of gene pools, environments, cultures, etc.) may also be included into it to some degree, the PERM has not yet been devised to address such issues clearly (see, e.g., Laland, Odling-Smee, \& Feldman, 1996, 1999). This chapter is thus at this point not so much concerned with evolutionary mechanisms (e.g., migration, selection, drift, etc.) although it will be important to consider evolutionary, cultural, and behavioral ecological theorization, methods, and findings in the future. Moving forward, these topics (see, e.g., Kendal et al., 2011) should be addressed an eco-evo-devo perspective adopted (e.g., Gilbert, Bosch, \& Ledón-Rettig, 2015).
} 
1582 environment (alloplastic mechanisms), or both at once or in rapid succession. The

1583 person $\rightarrow$ environment NMs in Figure 9 concern alloplastic mechanisms where something

1584 happens to the environment as a function of the person. As can be seen in Figure 9, the evaluation 1585 of an environment may result in either of two broad person $\rightarrow$ environment NMs: "maintaining"

1586 versus “terminating." Specifically, Rauthmann (2016) hypothesized that perceiving the

1587 environment as more pleasant than unpleasant should result more likely in maintaining, while

1588 perceiving it as more unpleasant than pleasant more likely in terminating. ${ }^{16}$ However,

1589 maintaining may also happen if the person cannot influence her/his environment and thus is

1590 rather passive. The maintenance person $\rightarrow$ environment NMs can fall into (1) remaining and just

1591 letting things happen (passive maintenance), (2) trying to preserve the environment as is (active

1592 maintenance), or (3) trying to get the most out of the environment (active maximizing). The latter

1593 may entail mild-intensity modifications and thus could also be grouped under the modification

1594 person $\rightarrow$ environment NMs instead of maintenance.

If the maintenance person $\rightarrow$ environment NMs are not desired, sensible, or possible, then

1596 termination person $\rightarrow$ environment NMs will (have to) be used. These can be either behavioral or

1597 mental. If they are mental, then a person changes something about the perceived environment.

1598 Technically (and as per Rauthmann et al., 2015a), this means persons change something about

1599 themselves, namely, their own perception of the environment (re-construal). However, the object

1600 of such re-construal still concerns the environment, meaning that the perceived but not actual

1601 environment is calibrated to oneself. As such, the re-construal mechanism is grouped here under

1602 person $\rightarrow$ environment NMs instead of being an autoplastic regulation mechanism (where the

\footnotetext{
16 This process is in actuality more complex as these environment perceptions will also be compared to how well one can handle the environment and thus also depend on persons' self-perceptions (e.g., of abilities, coping, etc.). Further, environments can have both pleasant and unpleasant (perceived) characteristics at once, and thus both need to be considered in conjunction with (or in relation to) each other simultaneously.
} 
1603 object is the self, not the environment). Some persons will engage in re-construal even if there is 1604 no necessity to do so; however, in most cases, re-construal is likely more of a secondary option 1605 when behavioral person $\rightarrow$ environment NMs - which focus on the actual (and not just perceived) 1606 environment - are not desirable, sensible, or possible. Those behavioral person $\rightarrow$ environment 1607 NMs can be termed "shaping" because a person somehow shapes the environment into a different 1608 version. Such shaping may be achieved by (1) willfully or inadvertently eliciting certain reactions 1609 (evocation), (2) leaving the current environment and entering a new one (selection), or (3) staying 1610 in the current environment and doing something with it (modification). Depending on the extent 1611 of purposeful changes made to the environment (mild-intensity: modulation; strong-intensity:

1612 creation), the pre-existing one may have slightly or moderately changed (modulation) or may 1613 have become an entirely new one (creation). In both selection and creation person $\rightarrow$ environment 1614 NMs, a new situation is the result, but by different means: Selection means exiting one 1615 environment and going into another one that is already there, while creation entails actively 1616 shaping an existing environment to become a new one. Of course, in daily life and across the 1617 lifespan, multiple person $\rightarrow$ environment NMs may be used or combined, even at the same time 1618 and perhaps even in the service of each other (i.e., there can also be correlations, interactions, fits, 1619 and transactions among and between navigation mechanisms).

$1620 \quad$ 5.2.1.2 Empirical Evidence. The different person $\rightarrow$ environment NMs in Table 9 and 1621 Figure 9 have not been systematically studied in unison, that is, how all of them operate on 1622 actualgenetic and ontogenetic timescales. Indeed, a grave limitation of most studies examining 1623 person-environment transactions is their inference, rather than actual measurement, of the past or 1624 current operation of person $\rightarrow$ environment NMs. For example, researchers could (and have) 1625 interpreted monodirectional person $\rightarrow$ environment transaction patterns (see 9-12 in Figure 8) as 
1626 evidence for selection effects: some prior traits (p) may predict later niche characteristics (e)

1627 (e.g., personality traits at age 18 predict work characteristics at age 26: Roberts, Caspi, \& Moffitt,

1628 2003). Such a conclusion can be warranted if one knows that the niches have changed (e.g.,

1629 persons changed jobs), but then selection may still be entangled with evocation, modulation, or

1630 creation because persons also shape and craft their niches in addition to picking them (e.g.,

1631 vocations, jobs: Holland, 1959, 1987; Wrzesniewksi, 2001). Similarly, different environment $\rightarrow$

1632 person transaction patterns (see 13-16 in Figure 8) have been interpreted as socialization effects

1633 (e.g., Denissen et al., 2014). ${ }^{17}$ However, in all of these instances possible explanatory

1634 mechanisms have been reduced to only one category (e.g., selection) and inferred from a pattern

1635 of findings (e.g., that prior person variables predict later environment variables). Both of these

1636 aspects are problematic.

The operation of alternative mechanisms cannot be ruled out that easily. For example,

1638 what may often seem like selection could easily also be evocation, modulation, or creation. After

1639 all, often certain trait levels may predict certain characteristics levels (see "contact" in

1640 Rauthmann et al., 2015c), but such observations do not allow firm conclusions regarding

1641 explanatory mechanisms. However, there are ways to tease apart some of the

1642 person $\rightarrow$ environment NMs even without measuring them explicitly. For example, under the

1643 condition that persons remain in the same niches (i.e., no ontogenetic selection effects; e.g.,

1644 staying in the same job at the same organization), then showing that person characteristics levels

1645 (e.g., Big Five traits) predict changes in environment characteristics levels (e.g., in a job) across

1646 time can signify transformative person $\rightarrow$ environment NMs, such as re-construal, evocation,

1647 modulation, or creation. There is research that suggests such transformative power of traits

\footnotetext{
17 These do not concern person $\rightarrow$ environment NMs, but navigation mechanisms for environment $\rightarrow$ person calibrations.
} 
1648 shaping niches (e.g., Sutin \& Costa, 2010; Wille, Beyers, \& de Fruyt, 2012) and of persons

1649 crafting their jobs and work according to their goals, values, and abilities (e.g., Wrzesniewski,

1650 2001). However, most studies have used self-reports for assessing both person and environment

1651 variables. Thus, it is not clear whether the underlying person $\rightarrow$ environment NMs have targeted

1652 the perceived environment via mental mechanisms (re-construal), the actual environment via

1653 behavioral mechanisms (evocation, modulation, creation), or both. A remedy is to use different

1654 data sources for measuring person and environment variables (see Denissen et al., 2014 for an

1655 example). When perceptions of environments can be differentiated from the exposure to actual

1656 environments, then it seems so far that persons' unique or idiosyncratic construals of daily

1657 situations $(\mathbb{E})$ are more strongly tied to personality traits $(\mathbb{p})$ than the exposure to or contact with

1658 certain situations (Abrahams, Rauthmann, \& De Fruyt, in prep.; Bolger \& Schilling, 1991; Hong,

1659 Chan, \& Lim, 2020; Rauthmann et al., 2015c).

Different person $\rightarrow$ environment NMs also cannot be disentangled easily because they are

1661 rarely if ever measured at all. The operation of a person $\rightarrow$ environment NM is usually post hoc

1662 inferred solely from a certain pattern of findings (e.g., see discussion of "selection effects"

1663 above). Because there can be multiple explanations, such inferences are often too weak to

1664 warrant the firm conclusion that one certain person $\rightarrow$ environment NM can explain the person-

1665 environment transaction pattern. Worsening matters is that the environment variables have often

1666 also not been assessed in a proper way (see Rauthmann et al., 2015a for a through discussion),

1667 that is, they often reflect actually person variables either conceptually (e.g., job satisfaction is a

1668 person variable although it pertains to a job) or by research design (e.g., the perception of an

1669 environment characteristic by one person is just a perception, not a "true" environment variable;

1670 for details, see Rauthmann et al., 2015a). Thus, any person-environment transaction with such 
1671 problematic measurement of the environment actually just represents a person-person transaction

1672 and can also inflate the associations found via common-method variance. Luckily, the

1673 measurement of situation information has progressed (e.g., Rauthmann, Horstmann, \& Sherman,

1674 2020) so that this aspect of the problem will hopefully cease to exist soon (see also 6.1 Taking

1675 Environment Assessment Seriously). A direct remedy to the "missing mechanisms problem" is

1676 obviously to actually measure the person $\rightarrow$ environment NMs (or any other type of navigation

1677 strategy; see Table 8 for all calibrations) and model their impacts (mediational or moderational,

1678 depending on the theory used) on the person-environment transaction patterns found.

1679 Unfortunately, there do not seem to be any validated and widely used measurement systems of

1680 person $\rightarrow$ environment NMs available so far (see also 6.5 Conceptualizing, Measuring, and

1681 Studying Calibrations and Navigation Mechanisms later).

1682 One of the first attempts to directly measure person $\rightarrow$ environment NMs was conducted in

1683 the "Narrative Dataset" by Ryne Sherman (see Brown, Blake, \& Sherman, 2017; Rauthmann \&

1684 Sherman, 2016). Two hundred and ninety-eight participants wore narrative clips (life-logging

1685 devices) that took pictures of persons' everyday lives every few seconds (. In a later laboratory

1686 session, participants segmented their entire photo stream into different situations. Among other

1687 things, they then also indicated in a forced-choice format why a situation had changed, using five

1688 person $\rightarrow$ environment NM options. Of all situations reported $(k=5,280)$, the following

1689 percentage changed because of a certain person $\rightarrow$ environment NM (in descending order, with 1690 items in parentheses): $46.6 \%$ for selection (I left the situation for another situation), $23.9 \%$ for

1691 manipulation (I did something to intentionally change the situation), $13.8 \%$ for construal (My

1692 perceptions or goals in the situation changed), $11.0 \%$ for evocation (The situation change was

1693 beyond my control), and $4.7 \%$ for Other (a leftover category if neither of the other four categories 
1694 applied). Thus, apparently persons report considerable agency over their daily situations and their changes, suggesting that person $\rightarrow$ environment calibrations especially with the navigation 1696 mechanisms of selection and manipulation (a mixture of modulation and creation) may be pervasive: together, they explain changes for $70.5 \%$ of situations.

person $\rightarrow$ environment NMs and thus provide first direct empirical evidence for their operations,

1700 there are also glaring limitations. First, a relatively undifferentiated person $\rightarrow$ environment NM

1701 measure was used that was created in an ad hoc fashion (after all, there was nothing else to use).

1702 It did not include all person $\rightarrow$ environment NMs (e.g., it focused only on terminating but not on

1703 maintaining because the task was to study situation change), it meshed different ones (e.g.,

1704 modulation and creation are blended into one), and it only allowed a forced-choice among the

1705 person $\rightarrow$ environment NMs. Regarding the latter issue, it stands to reason that different

1706 person $\rightarrow$ environment NMs do not have to be mutually exclusive but could actually be used

1707 simultaneously or at least in rapid succession (e.g., modulation and re-construal in conjunction).

1708 Thus, there need to be more systematic efforts to conceptualize and measure

1709 person $\rightarrow$ environment NMs.

1710 Second, all data are self-reports only from the persons in the situations. While there is

1711 intuitive appeal to this approach (after all, those persons in their own situations could be viewed

1712 as "experts"), the sole reliance on what persons report about their situations changing is

1713 problematic. For example, they may suffer from different self-serving, memory, or other biases

1714 that could explain the strong observed levels of person agency over situations: persons could

1715 erroneously conclude that a situation has changed because they think (or wished) they used

1716 selection or manipulation, but they may be (for different reasons) blind to other reasons (e.g., 
1717 evocation). Thus, it is important to measure person $\rightarrow$ environment NMs not just via self-reports

1718 but ideally also observationally or behaviorally (i.e., what persons actually do to/with their

1719 environments). This has been difficult so far, but novel smartphone and machine sensor

1720 technology may alleviate this problem and make such measurements possible in a continuous,

1721 fine-grained, and unobtrusive manner (e.g., Blake et al., in press; Harari et al., 2017, in press;

1722 Harari et al., Chapter 29; Miller, 2012; Pérez-Edgar, MacNeill, \& Fu, 2020), of course keeping

1723 legal and ethical issues in mind.

$1724 \quad$ 5.2.2 Navigation Mechanisms for Environment $\rightarrow$ Person Calibrations. Table 8 also

1725 lists six macroscopic "mechanisms" of how environments may calibrate persons. This list is

1726 neither exhaustive nor systematic, and in reality, there is likely a myriad of different mechanisms.

1727 However, the list offered here reflects a first attempt at tying together different streams of

1728 research under the umbrella of belonging to navigation mechanisms for environment $\rightarrow$ person

1729 calibrations (i.e., environment $\rightarrow$ person NMs). Additionally, most of the environment $\rightarrow$ person

1730 NMs listed are not just "inverted" forms of person $\rightarrow$ environment NMs. Indeed, it would be

1731 possible to conceptualize environments as "affording" maintenance, re-construal, evocation,

1732 selection, modulation, or creation and thus making persons do these things. While

1733 straightforward, this somewhat diminishes persons' agencies and also is a conceptual cop-out:

1734 There is no reason to believe that environment-based mechanisms have to follow the same molds

1735 as person-based mechanisms, even if that would be easier to process and be more elegant. Thus, I

1736 explicitly tried to compile environment $\rightarrow$ person NMs that would be largely unique to

1737 environments' agency over persons, launching persons into certain presses that would impact

1738 their mental and behavioral functioning. It is beyond the scope of this chapter to also detail each

1739 of the environment $\rightarrow$ person NMs, but I hope that this non-exhaustive list could serve as a viable

1740 starting point to think about how environments draw, repel, and shape persons. 


\section{Recommendations and Future Directions}

The preceding sections attempted to organize and review the scattered literatures on person-environment relations. But where to go from here? Below, I compile ten suggestions and recommendations on how to proceed.

\subsection{Taking Environment Assessment Seriously}

To examine the phenomena and paths in the PERM, psychometrically optimized assessments of person and environment variables are vital. While trait variables have received a tremendous amount of attention and spurred multiple well-validated and widely used assessment instruments, the assessment of person states (e.g., Horstmann \& Ziegler, 2020) and of any environment variables still lags behind (Rauthmann et al., 2015a). Recently, there have been some efforts to remedy this in the study of psychological situations (Rauthmann \& Sherman, 2020), with newer research aiming to measure perceived or psychologically active characteristics of situations by judgments of persons (for an overview of taxonomic systems and measures, see Rauthmann et al., 2020).

However, the assessment of environment variables has further issues that need to be resolved. First, there are exciting developments for multi-trait multi-method multi-occasion data modeling (e.g., Geiser et al., 2015, Chapter 35; Hintz et al., 2019) that present powerful dataanalytical strategies to model many of the PERM's variables and effects on a latent basis. Nonetheless, latent occasion variables often remain truly latent, simply inferred from the measurement of variables at different occasions (e.g., personality states) without the actual measurement of environment variables. This is akin to much of twin-study research that has decomposed phenotypical variance into genetic and environment contributions but failed to account for the actual environment variables (which, unfortunately, are usually not directly measured) that may explain the shared or non-shared environment variance. These kinds of 
1765 models had to historically cope with the fact that almost no measures of environment variables

1766 were available, so they have proceeded without incorporating any manifest environment

1767 indicators. To fully understand person-environment relations, however, both person and

1768 environment variables need to be assessed with great care. Thus, manifest environment variables

1769 need to be directly sampled in future research (see Zapko-Willmes, Riemann, \& Kandler, 2018,

1770 for an example of a twin family study approach with multiple assessments of the person variable

1771 “authoritarianism” and the environment variable "differential parenting”).

1772 Second, if psychological characteristics of environments (especially E) - as manifest

1773 variables - are to be assessed (see Rauthmann et al., 2015a,b for a rationale for this approach),

1774 then it is important to not just use one person's perception of a situation. Indeed, such a

1775 perception is actually merely a person variable (namely, P) and not an environment variable. To

1776 obtain actual environment variables from ratings, it is necessary to form composite scores from

1777 several raters - ideally even of different perspectives (in situ: from within a given situation; juxta

1778 situm: from bystanders who are not directly affected; ex situ: from written texts, audio, or video

1779 footage). Such a multi-source composite will better approximate an actual environment variable

1780 instead of capitalizing on a person variable (i.e., one person's perception). Specifically, such a

1781 variable will likely represent the shared variance or consensus among different raters (Rauthmann

1782 \& Sherman, 2019; Zapko-Willmes et al., 2018).

\section{6.2 Employing Appropriate Research Designs}

1784 To capture the effect paths outlined in the PERM and provide robust estimates of them, 1785 real-life multi-method multi-situation multi-time multi-group (RL-MM-MS-MT-MG) designs

1786 should be used (see Rauthmann et al., 2015a) that enable testing multiple constraints on 1787 generality (Simons, Shoda, \& Lindsay, 2017) and thus the robustness of findings. First, those

1788 designs should approximate real life (RL) as well as possible. This means that both field and 
1789 laboratory data can be sampled (Wrzus \& Mehl, 2015), but in each instance there should be

1790 ecologically valid settings involved which may require the use of novel technologies to truly do

1791 so (e.g., using virtual and augmented reality in the laboratory and wearables in the field; Blake et

1792 al., in press; Harari et al., Chapter 29; Riva et al., 2016). The underlying idea is that findings

1793 should generalize to persons' everyday lives and be applicable there. This is especially important

1794 for experimental designs (often conducted in a laboratory) because they usually eradicate

1795 preexisting person-environment correlations. The person-environment relations found, then, are

1796 different ones than those in the field where there are natural person-environment correlations.

Second, designs should involve multi-method data (MM) from different sources (e.g., see

1798 the BIOPSIES mentioned earlier for trait and state assessments; Rauthmann, 2017). This is

1799 especially important when sampling environment variables (see 6.1 Taking Environment

1800 Assessment Seriously), but should also be taken into account when sampling person variables.

1801 The shared variance among the different data sources or methods may better approximate the

1802 latent variables to be measured, and differences between methods (or their uniquenesses) may be

1803 of interest (Geiser et al., 2015; Hintz et al., 2019). The underlying idea is that findings should

1804 generalize across different methods used.

1805 Third, designs should sample person and environment variables in multiple situations

1806 (MS) per person and not just in one or a few. If the situations (measurement occasions) have been

1807 sampled representatively for each person in their daily life, then this will be especially beneficial

1808 to sampled momentary person (P) and environment variables (E). Further, having several

1809 measurement occasions allows estimating density distributions of the measured variables and

1810 thus quantifying intra- and inter-individual variabilities (Fleeson, 2001). The underlying idea is

1811 that such designs can quantify the short-term fluctuations in effects and also examine to what

1812 extent effects reside at the within- or between-person levels (as measurement occasions will be

1813 nested within persons). 
Fourth, designs should use multiple time-points (MT) to sample person and environment

1815 variables. The time-points would ideally be far apart (e.g., months or years as intervals), and at

1816 each time-point multiple measurement occasions can be sampled (measurement burst design:

1817 Sliwinski, 2008). Such designs allow quantifying the levels of stability vs. change in all variables

1818 and effects uncovered and may be used to answer developmental or lifespan questions (e.g.,

1819 Freund \& Baltes, 2000; Haase et al., 2013). The underlying idea is thus that the temporal

1820 robustness of findings can be examined (i.e., the extent to which they generalize across time).

$1821 \quad$ Lastly, designs should make use of multiple groups of persons and/or environments

1822 (MG). Groups of interest may vary with research hypotheses and by discipline, but person groups

1823 could be distinguished, for example, by sex/gender, education, (un-)employment, mental health

1824 (healthy vs. disordered), zygosity (monozygotic vs. dizygotic), postal zip code, or even country.

1825 Environment groups (classes) could be distinguished, for example, by categories such as

1826 "everyday vs. extreme", "hazardous vs. non-hazardous", "leisure vs. work", etc. The underlying

1827 idea is that findings may or may not generalize across (perhaps qualitatively) different sets of

1828 persons and environments, and commonalities and differences can be examined closely.

Taken together, a full RL-MM-MS-MT-MG design sampling person and environment

1830 variables in ecologically valid settings with multiple methods at multiple measurement occasions

1831 for multiple time-points in multiple groups of persons and/or environments would be a most

1832 powerful design. Such a design would permit estimating several forms of robustness,

1833 replicability, or generalizability at once. However, even "lesser" designs (e.g., RL-MM-MS:

1834 Rauthmann et al., 2015c) can also permit relatively strong conclusions about person-environment

1835 relations. Nonetheless, as a general recommendation, more than one and ideally as many of the

1836 five design requirements as possible (RL, MM, MS, MT, MG) should be combined in one design. 


\subsection{Zooming into Processes, Sequences, and Cycles}

This chapter is only concerned with relatively abstract classes of processes, such as ontogenesis and actualgenesis or manifestation and accretion. However, how exactly and which different processes unfold and to what extent, when, and how they concatenate to sequences or even cycles has been omitted. Nonetheless, to study personality dynamics, processes, and functioning, it will be important to zoom in on these neglected topics (cf. Baumert et al., 2017; Blum et al., Chapter 2; Fleeson, 2017; Wrzus \& Roberts, 2017). This will hopefully lead to a richer understanding of person-environment relations. Indeed, it will likely be necessary to adjust the generic PERM in Figure 2 in certain ways to suit dynamic personality research even better. An example of this is given in Figure 6 as well as in Box 5 and Figure 10. Specifically, in Figure 6, a process model of person-environment fit and its consequences is shown - effectively acting as a magnifying glass on the macroscopic effects captured in the PERM. In Box 5 and Figure 10, a corresponsive pattern of personality development is exemplarily traced within a modified PERM. The PERM should be seen as a flexible framework that can, and should, be modified according to different research aims, substantive research questions, and specific hypotheses. One particularly fruitful avenue will be to use the PERM for a more dynamic understanding of personality and its development.

- Box $5-$

- Figure $10-$

\subsection{Explanation vs. Prediction and Causality}

Person-environment correlations, interactions, fits, and even transaction patterns may be used to explain or predict certain outcomes, such as diversity in personality expressions (in different populations of persons and environments), continuity and change in personality 
1860 development, success at work, longevity, or intra- and interpersonal adjustment. Notably, it is

1861 important to distinguish explanation from prediction and appreciate the tension between them.

1862 For example, according to Yarkoni and Westfall (2017), there is a trade-off between explanatory

1863 models that are supposed to be simple, theoretically elegant, and contain abstract principles

1864 versus predictive models that are more complex, not theoretically grounded, and pragmatically

1865 optimize the prediction of an outcome by any means. Especially with the advent of machine

1866 learning and "Big Data" approaches (Bleidorn \& Hopwood, 2019), prediction has moved nearer

1867 into the grasp of personality science. Nonetheless, explanation is still the predominant concern in

1868 psychology. This has led to a wide-spread acceptance of experiments (i.e., manipulating an

1869 independent variable to gauge its causal effects on a dependent variable, while controlling,

1870 standardizing, or randomizing many other variables that could have influences) to establish

1871 causality (Shadish, Cook, \& Campbell, 2002). In this view, explanation ("If X explains Y, then X

1872 causes $\left.\mathrm{Y}^{\prime \prime}\right)$ is in fact different from prediction because predictions are still possible even without

1873 predictors causing the criterion variables. Causality has been traditionally a thorny issue in

1874 psychology, but research on personality (Costantini \& Perugini, 2018; Lee, 2012; Mõttus \&

1875 Kandler, 2018) and person-environment relations (Asendorpf \& Motti-Stefanidi, 2018) is of

1876 course also interested in making causal claims. Experimental studies are likely to remove

1877 naturally occurring person-environment correlations and thus may lead to artificial effects. On the

1878 other hand, non-experimental studies may contain too much "noise", spurious effects, or wash

1879 out otherwise interesting effects.

1880 In keeping with the requirements of RL-MM-MS-MT-MG designs that include time-

1881 sensitive information, cross-lagged analyses can be performed to establish temporo-sequential

1882 chains that could approach causality (Gollob \& Reichardt, 1987). However, they only establish a

1883 certain direction of effects which represent Granger causality (Granger, 1969). In the absence of 
1884 coupling such designs with experimental manipulation, many would argue that only quasi-

1885 causality can be established. However, more sophisticated frameworks for establishing causality

1886 exist (e.g., Pearl, 2009; Rubin, 1974; Spirtes, Glymour, \& Scheines, 2001), and these can and

1887 should also be applied to PERM effects. For example, several parts of the PERM may be

1888 translated into directed acyclic graphs (for an accessible discussion and demonstration, see Lee,

1889 2012). Further, conceptual rigor and proper operationalizations as well as theories and sound

1890 hypotheses are needed in the first place. For example, Lee (2012) alerts us that "[a]

1891 developmental process may predetermine Y well before $\mathrm{X}$, even if X is measured first” and

1892 "[t]hus, the soundness of any causal conclusion depends on both conforming data and the

1893 correctness of the requisite assumptions" (p. 373). Taken together, for person-environment

1894 relation research to move forward in both explanation and prediction as well as establishing

1895 causalities, it will be important to prudently use and firmly interlock theory, research designs, and

1896 sophisticated data-analytical strategies.

6.5 Conceptualizing, Measuring, and Studying Calibrations and Navigation Mechanisms

The NMs in Figure 9 have been identified partially in and scattered throughout different

1899 literatures (see Table 9). Although different kinds of NMs should be distinguished (Table 8),

1900 arguably among the more interesting ones for personality researchers will be the mechanisms for

1901 person $\rightarrow$ environment calibrations. This chapter only provided a very general roadmap towards

1902 structuring and potentially distinguishing those NMs (see 5.2.1.1 A Systematic Account). More

1903 conceptual clarity and theorizing are needed. Additionally, there are to date no validated

1904 measurement tools for assessing (inter-individual differences in the use of) NMs available (cf.

1905 Brown et al., 2017 for an ad hoc, forced-choice, and self-report measure). Thus, future research

1906 should develop methodologies for assessing the NMs from different data sources (e.g., self-

1907 ratings, peer-ratings, behavioral observation, actual behavioral measurement) in a valid and 
1908 reliable manner. Such measurement can then help address several substantive research questions

1909 that remain unanswered so far. For example, future empirical research - based on better

1910 conceptualizations and psychometrically validated measures - could investigate for the NMs: (1)

1911 the extent of inter-individual differences in their habitual or preferential use; (2) to what extent

1912 they are consciously and deliberately used; (3) which role underlying needs and motivations play;

1913 (4) how time, energy, and resources are allocated to perform them and which decision rules

1914 persons use (e.g., when to remain vs. terminate a situation); (5) which opportunities and obstacles 1915 govern their usage; (6) their proximal and distal functional values and adaptive purposes (why, 1916 when, and for what are they used?); (7) their styles of utilization (how or in what manner are they 1917 used?); (8) their nomological networks (causes/antecedents, concomitants, and consequences in 1918 actualgenetic and ontogenetic contexts); (9) if, how, and when they contribute to accentuating, 1919 changing, stabilizing, or destabilizing inter-individual differences; (10) levels of genetic variance 1920 at different ages; (11) stability vs. change and trajectories across the lifespan (as well as inter1921 individual differences in these); and (12) if, when, and how their repeated actualgenetic use may 1922 coalesce into niche picking, crafting, and fitting on an ontogenetic scale.

\section{6.6 Distinguishing Different Types of Person-Environment Fit}

1924 Because profile-oriented and variable-oriented person-environment fit analyses capture 1925 distinct phenomena (in each case "fit" is quantified differently and captures different aspects), 1926 researchers need to specify exactly which kind of "fit" they are examining, why they refer to "fit" 1927 in the first place, and which data-analytical strategies they have exactly used. In any case, person1928 environment fit will not be a monolithic or static phenomenon in people's lives. As this chapter 1929 hopefully made clear, there can be multiple forms of fit, depending on the types and content of 1930 variables used as well as the levels of abstraction. For example, one could conceptually envision 1931 a hierarchical model of person-environment fit with a global and undifferentiated fit factor $f_{\mathrm{g}}$ at 
1932 the apex and then several more domain-specific fits $f_{\mathrm{s}}$ at lower levels (e.g., fit to culture, work

1933 team, company, sports, education environment, friends, romantic partner, a specific restaurant,

1934 etc.). Additionally, each of these types of fit may be accompanied by other dynamics and

1935 processes (De Cooman et al., 2019; for a suggested general starting point, see Figure 6). Taken

1936 together, it is of crucial importance to clearly specify what kind of person-environment fit is

1937 studied and what its scope shall be as the nomological networks (with causes/antecedents,

1938 concomitants, and consequences) of one fit may not necessarily generalize to others fits.

1939 6.7 A Transactional Perspective on Traits and Personality

1940 Attentive readers may have noticed that neither the exact relations between person traits

$1941(\mathbb{P})$ and states $(\mathbb{P})$ nor their respective exact definitions have been made clear in this chapter (save

1942 for Figure 10 that served only for illustration purposes; see Box 5 for details). This was done in a

1943 deliberate attempt to avoid several thorny issues in the definitions of traits and states and thus to

1944 focus more on the person-environment relations. However, moving forward, traits and states do

1945 require being specified better. For example, a systems-theoretical approach (e.g., Rauthmann,

1946 2015a; Sosnowska et al., 2019) might model the concurrent and temporal structures and

1947 dynamics of circumscribed PERM systems. To the extent that some structures and dynamics

1948 show a non-chance patterning and are recurrent, these may together represent a "trait." Thus, a

1949 trait would be an emergent phenomenon arising from networks or systems of functionally related

1950 variables (for similar views, see Cramer et al., 2012) rather than a separate entity in the system.

1951 Such a transactional perspective has several implications for how we understand

1952 personality as a whole as well as traits and person dynamics in particular. First, here traits "exist"

1953 between persons and environments as well as within their structural and dynamic relations. This

1954 view is not new, however, and has already been espoused by early interpersonal theorists. For

1955 example, for Sullivan (1953) "personality is the relatively enduring pattern of interpersonal 
1956 situations that characterize a life" (pp. 110-111). New treatises of normal and abnormal

1957 personality functioning also subscribe to such a view (Hopwood, 2018). Indeed, there is also

1958 some empirical evidence that traits manifest in the ways in which persons engage with their

1959 environments (e.g., Srivastava, Angelo, \& Vallereux, 2008), and traits could also be

1960 conceptualized as tendencies towards establishing certain situations.

Second, by knowing in which kinds of situations or environments a person habitually is

1962 and how she/he navigates those situations, it should be possible to know the person better and

1963 derive accurate personality judgments. For example, it has been argued that "one's choices of the

1964 settings in which to live one's life ... may reflect features of one's conceptions of self, one's

1965 characteristic dispositions, one's attitudes and values, and other attributes of personality" (Snyder

1966 \& Ickes, 1985, p. 915). Further, early theorizing has posited that the self is manifest in

1967 “symbolic" environments, such as one’s house or places (e.g., Cooper, 1974; Proshanksy, Fabian,

1968 \& Kaminoff, 1983). An empirical example is Gosling et al.'s (2002) study that examined to what

1969 extent judges' trait ratings correlated with targets' self- and peer-ratings based solely on viewing

1970 the targets' bedrooms or offices. It rests on the idea that persons' behaviors manifest in imprints

1971 in their offline and online environments and leave behavioral residues that can be judged by

1972 persons (e.g., Gosling, Gifford, \& McCunn, 2013; Gosling et al., 2008; Graham, Sandy, \&

1973 Gosling, 2011; Kosinski, Stillwell, \& Graepel, 2013; Mehl, Gosling, Pennebaker, 2006; Vazire \&

1974 Gosling, 2004). Thus, environmental niche variables e that are an effect of single or repeated

1975 person states PS, which in turn are instances of person traits ps, can be used to infer ps.

1976 Lastly, a transactional perspective resolves the apparent dichotomy between persons and

1977 situations (Fleeson \& Noftle, 2008a,b; Hogan, 2009) because both always need to be considered

1978 in unison. This perspective further underscores how silly the person-situation debate actually was

1979 (though it was productive in terms of pushing researchers to confront thorny issues and 
1980 concretize their thoughts; see Kenrick \& Funder, 1988). Indeed, to explain complex phenomena

1981 such as that "[p]eople create, seek out, or otherwise gravitate to environments that are compatible

1982 with their traits" (Hampson, 2012, p. 318) or that persons show considerable amounts of within-

1983 person variability of behavior (Beck \& Jackson, Chapter 4), it is important to consider persons

1984 and environments jointly. To give an example where such joint consideration is especially useful,

1985 consider Ickes, Snyder, and Garcia’s (1997, p. 166) assertion that “[persons'] preferred

1986 environments provide opportunities for personal dispositions to be manifested and reinforced"

1987 and that "[o]nce individuals are in their chosen situations, their words and actions are genuine

1988 reflections of their personalities." Several hypotheses can be derived here, such as that (1)

1989 persons should have cognitive-affective preferences towards some environments but not others;

1990 (2) persons may be motivated to gravitate towards preferred, and shun non-preferred

1991 environments; (3) selected environments should entail higher congruence between traits and

1992 manifested state enactments (i.e., associations between persons' traits and states are moderated by

1993 their environment selection); (4) those environments should additionally serve to reinforce, or

1994 stabilize, the state enactments, thus leading to higher state-to-state consistency; and (5) persons

1995 may perhaps pick up on their "genuine" trait-state congruence in those environments and thus

1996 feel authentic (but see for counterevidence Cooper et al., 2018). These hypotheses can only be

1997 tested if we subscribe to a more transactional perspective as well as sample and model both

1998 person and environment variables at the same time.

$1999 \quad 6.8$ Formalization

To avoid even more complexity and provide a more accessible treatise of person-

2001 environment relations, this chapter did not engage in full mathematical formalization of all

2002 functions or elements and relations depicted in Figure 2. However, there is already recent

2003 theorization that moves towards more formalization. For example, Todd and Gigerenzer (2020) 
2004 propose in their ecological rationality approach that person variables represent an adaptive 2005 toolbox (AT) of heuristics that can be used to make decisions and take action (B) with respect to 2006 certain environmental settings $(\mathrm{E})$, thus yielding: $\mathrm{B}=f(\mathrm{AT}, \mathrm{E})$. They exemplify their approach 2007 with trust in doctors, parental investment in middle-born children, and burglars choosing a 2008 property to break in. The ecological rationality approach has the aim to concretize the often 2009 abstract "function" relating person variables (adaptive decision heuristics) and environment 2010 variables. Further, Wood, Spain, and Harms (2020) have presented a functional approach to 2011 representing the interplay of persons, situations, and behaviors in a formalized way using 2012 functional fields (see also Wood, Chapter 24; Wood et al., 2019), integrating ideas from Lewin’s 2013 (1943) field theory and modern causal modeling (Pearl, 2009). Functional units may exert causal 2014 forces, and these can be represented in vector spaces. Indeed, such thinking has already been 2015 espoused quite early, as in Lewin (1943) but also in Raymond Cattell's impressive body of work 2016 (see Boyle, 2019 for an overview). Further, dynamic systems theory also provides a rich and 2017 mathematically formalizable framework to understand person-environment systems (Allen, 2018 Sylaska, \& Mayer, Chapter 26; Rauthmann, 2015a; Richardson, Dale, \& Marsh, 2014;

2019 Scheinerman, 1996; Sosnowska et al., 2019; Micheals, Vallacher, \& Nowak, Chapter 38). Lastly, 2020 stronger formalization is a requirement for computational modeling and proof-of-principle 2021 hypothesis testing (Fraley \& Roberts, 2005; Mõttus, Allerhand, \& Johnson, 2020; Read et al., 2022 2010) where person and environment variables as well as their relations need to be carefully 2023 operationalized and according parameters and models formulated. For example, Fraley and 2024 Roberts (2005) concluded based on simulated dynamic models that reactive, evocative, and pro2025 active "transactional processes serve to amplify the degree of stability in individual differences" 2026 and "also allow persons, constants, and environments to function as a coherent system of 2027 elements that mutually sustain and reinforce one another" (p. 68). Recently, Mõttus, et al. (2020) 
2028 have also provided simulated examples of person-environment relations where accumulated

2029 situational experiences impacted the variance in personality characteristics across time and gave

2030 rise to structural personality factors.

2031 Formalization is conditional upon conceptual clarity and rigor. Currently hampering

2032 stronger formalization, however, are terminological inconsistencies and conceptual blurriness that

2033 are pervasive throughout the literature. ${ }^{18}$ Moreover, some phenomena do not even have clear

2034 labels (yet). For example, person variables could be examined as states or traits. The entirety of

2035 all traits may represent, or at least approximate, personality. On the other hand, we have no clear

2036 label for the entirety of a person's momentary states. Can we speak of "stat(e)ality"? Moreover,

2037 environment variables can be defined for the shorter-term (situations) and longer-term (niches).

2038 However, how are their respective variables to be labeled (e.g., Blum \& Schmitt, 2015)? Should

2039 situations have states, and niches traits? Or could both a situation or a niche have either a state

2040 (when only one person judges their characteristics) or a trait (when there is cross-person

2041 consistency, or consensus, in judging their characteristics), respectively? ${ }^{19}$ And how is the

2042 entirety of state or trait characteristics of environments (situations or niches) referred to? Can we

2043 speak of "situationality", "nichality", or "environmentality" in some or either one of these? These

2044 are issues that would need to be resolved in the future to move towards a more unified,

2045 consensual language that will help set the basis of a formalized and precisely communicable

2046 perspective on person-environment relations.

\section{6.9 Theory, Multidisciplinary Integration, Replication, and Collaboration}

2048 Identifying meaningful and robust effects is a core aim of any scientific endeavor. For

2049 person-environment relations, previous empirical research has shown that distinguishing between

\footnotetext{
18 And unfortunately likely also even within this very chapter!

${ }^{19}$ I thank Gabriela Blum for bringing up this idea in a personal email communication (May, 2019).
} 
2050 conceptually more and less plausible relations makes a difference: effects for conceptually

2051 plausible pairs of person and environment variables appear to be stronger and more robust vis-à-

2052 vis implausible ones (Horstmann et al., 2020; Rauthmann et al., 2016). This underscores that a

2053 priori hypothesis generation and theory will be important. However, there are barely any strong

2054 theories explicitly for person-environment relations. For example, this chapter did not present a

2055 theory, but rather a formal meta-model or framework within which to systematically couch

2056 different phenomena and effects. This may not be too problematic as, in fact, the argument could

2057 be made that person-environment relations research still needs to be somewhat atheoretical at this

2058 point: Exploratory and descriptive work with large datasets and broad questions should, over

2059 time, lead to more refined hypotheses and theory-building. However, we still do not have enough

2060 of such work. Indeed, the PERM alerts us to several blind-spots that still need to be filled.

Once more systematic work with robust findings has been conducted, a major aim will be

2062 to integrate the insights gleaned and craft actual theories. Such synthesis will also entail utilizing

2063 synergies from multidisciplinary projects. The cross-talk between different disciplines (e.g.,

2064 genetics, behavioral ecology) that are also concerned with person-environment relations, but from

2065 different perspectives, will be immensely important going forward. For example, an eco-evo-

2066 devo framework (Gilbert et al., 2015) - which is also relevant to PERM phenomena - already

2067 demonstrates the utility and value of cross-fertilization between different disciplines to address

2068 complex questions. The resulting hopefully inter- or even transdisciplinary theories will then be

2069 generative in the sense that they make lacunas obvious, channel research efforts, and produce

2070 hypotheses for confirmatory testing. This, however, also requires different theories, approaches,

2071 and methods within psychology to be integrated. For example, this chapter has hopefully

2072 demonstrated that person-environment relations have been examined in several psychological

2073 disciplines (especially personality, social, developmental, evolutionary, genetic, applied, 
2074 environmental/ecological) - usually in a separate and insular manner. Particularly environmental

2075 and ecological psychology has seemed to embrace "transactionalism" where persons and

2076 environments are interdependent, the person-environment relation is the unit of analysis, and

2077 persons and environments can transform each other across time (e.g., Altman \& Rogoff, 1987;

2078 Gifford, 2007; Saegert \& Winkel, 1990; Stokols, 1995; Stokols, Clitheroe, \& Zmuidzinas, 2000;

2079 Stokols \& Shumaker, 1981; Walsh et al., 2000; Wapner, 1982). However, much of that work is

2080 barely integrated into common personality, social, and developmental psychological literatures.

2081 Additionally, there seems to be some rift between more basic and applied disciplines as they tend

2082 to also form distinct literatures (e.g., applications to occupational performance: Law et al., 1996;

2083 Strong et al., 1999; social work practice: German, 1981; leadership: Gammel, Chapter 43;

2084 psychopathology and psychotherapy: Ringwald et al., Chapter 11). Further, there are also several 2085 personality-psychological models concerned with dynamics and processes (e.g., PERSOC model:

2086 Back et al., 2011; KAPA: Cervone, Chapter 22; Cybernetic Big Five Theory: DeYoung, 2015;

2087 Whole Trait Theory: Fleeson, Chapter 21; Fleeson \& Jayawickreme, 2015; Jayawickreme et al.,

2088 2019; Five-Factor Theory: McCrae, 2019; McCrae \& Costa, 2008; socioanalytic and neo-

2089 socioanalytic model of personality: Hogan \& Roberts, 2000; Roberts \& Damian, 2017; Roberts \&

2090 Nickel, 2017; Roberts \& Wood, 2006; Cognitive-affective processing system: Mischel \& Shoda,

2091 1995, 1999, 2008; socio-genomic model of personality: Roberts, 2018; TESSERA model of

2092 personality development: Wrzus, Chapter 5; Wrzus \& Roberts, 2017) that would also need to be

2093 integrated with each other (cf. Quirin et al., in revision) and merged with a person-environment

2094 relations perspective. Altogether, it would be desirable to create one larger and strong "river"

2095 from the various parallel "streams" of literatures to study person-environment relations more

2096 holistically and in a unified manner. 
Any theories developed will have to integrate methodology, and any methodology (e.g., new technological advances, RL-MM-MS-MT-MG designs) needs to incorporate theory - in other words, both need to be balanced to yield synergies because each of them alone will not be sufficient to grasp the complex nature of person-environment relations. While methodologies including research methods and data-analytical strategies - are rapidly evolving, theory may currently not be keeping the pace enough. A solid exploratory phase is important for now, but researchers should already try to craft proto-theories or at least have bigger pictures in mind. Theories will have to rely on a good data basis as science is a cumulative process. Thus, 2105 insights need to be robust or we at least need to understand their constraints on generality 2106 (Simons et al., 2017). Towards this end, it will be important to employ RL-MM-MS-MT-MG 2107 designs and explicitly attend to the robustness, replicability, or generalizability of findings. This 2108 includes identifying contextual and moderator conditions that render established effects weaker or stronger. Moreover, many of the empirical findings surveyed in this chapter still await corroboration and replication. In other words, for most effects in the PERM, we simply do not

2111 have well-grounded knowledge yet. However, a strong cumulative knowledge base of replicable 2112 effects and a deeper understanding will, in turn, be essential in crafting good theories.

2113 Lastly, science is not only a cumulative, but also a cooperative endeavor. Studying 2114 person-environment relations may be time-consuming and require many resources. Thus, it is 2115 vital to start working towards multidisciplinary consortia that tackle large and complex questions 2116 with coordinated multi-site designs and sophisticated data-analytical strategies (for inspiration, 2117 see the entire Section III: Methods and Statistics of this handbook). This will be more economical 2118 and efficient than single and arguably more limited research projects. This recommendation is not 2119 to discourage well-crafted single research projects; rather, it should be understood as a call for 2120 more cooperation and cross-talk because that will also spur the cross-fertilization of ideas across 
2121 multiple psychological disciplines and beyond. A multidisciplinary consortium could further

2122 conceptually hone the crude concepts presented in this chapter and conduct cutting-edge research

2123 that might lead to a truly inter- and even transdisciplinary approach to person-environment

2124 relations.

\subsection{Working Towards Principles of Person-Environment Relations}

This chapter deliberately did not introduce any "principles" of person-environment

2127 relations just yet. While there are some findings that seem robust and may begin to materialize as

2128 principles (e.g., that construal may be tied more strongly to personality traits than other

2129 navigation mechanisms, that selection effects often seem to be ontogenetically stronger than

2130 socialization effects), I think it is still too early and thus premature to declare any principles.

2131 When Roberts and colleagues first formulated principles of personality development (e.g.,

2132 Roberts \& Wood, 2006), they could already look back at impressive amounts of work in a

2133 circumscribed domain of research. This is not yet the case with person-environment relations

2134 research that is scattered across different literatures. More importantly, however, there appears to

2135 be no overarching framework or theory guiding research on person-environment relations, and so

2136 it is difficult to integrate findings and distill a set of principles from robust and recurring patterns

2137 of insights (see 6.9). While the principles of personality development now effectively serve to

2138 integrate different findings on the continuity and change of personality and are thus regularly

2139 updated (e.g., Roberts \& Nickel, in press), no such thing exists for person-environment relations

2140 yet. My hope is that this chapter can represent a first step towards thinking more systematically

2141 about person-environment relations and locating different phenomena - with their studies and

2142 findings - within a common proto-framework that is still in flux and will likely change once

2143 more empirical findings arrive. In other words, hopefully this chapter and many other efforts can

2144 set the stage so that principles of person-environment relations can be chiseled out in the future. 
2152 being a special case of the former two. It is my hope that this chapter can be used as a starting

2153 point to think more systematically and deeply about person-environment relations as well as their

2154 causes, concomitants, and consequences in daily life and across the entire lifespan. 


\section{References}

Abdukadirov, S. (Ed.). (2016). Nudge Theory in Action: Behavioral design in policy and markets. Springer.

Abrahams, L., Rauthmann, J. F., \& De Fruyt, P. (in prep.). Person-situation dynamics in educational contexts: A self- and other-rated experience sampling study of teachers' states, traits, situations, and outcomes. Manuscript in preparation. See also osf.io/p642h

Abramowitz, J. S., Deacon, B. J., \& Whiteside, S. P. (2019). Exposure therapy for anxiety: Principles and practice. Guilford Publications.

Allport, G. W. (1937). Personality: A psychological interpretation. Holt, Rinehart, \& Winston. Allport, G. W. (1961). Pattern and Growth in Personality. Holt, Rinehart, \& Winston.

Altman, I., \& Rogoff, B. (1987). World views in psychology and environmental psychology: Trait, interactional, organismic and transactional perspectives. In I. Altman \& D. Stokols (Eds.), Handbook of environmental psychology (pp. 245-281). Wiley.

Arno, A., \& Thomas, S. (2016). The efficacy of nudge theory strategies in influencing adult dietary behaviour: A systematic review and meta-analysis. BMC Public Health, 16, 676.

Arslan, R. C., \& Penke, L. (2015). Evolutionary genetics. In D. M. Buss (Ed.), Handbook of Evolutionary Psychology Vol. 2: Integrations (2. ed., pp. 1047-1066). Wiley.

Asendorpf, J. B. (1990). The measurement of individual consistency. Methodika, 4, 1-23.

Asendorpf, J. B. (2020). Personality as a situation: A target-centered perspective on social situations. In J. F. Rauthmann, R. A. Sherman, \& D. C. Funder (Eds.), The Oxford Handbook of Psychological Situations. Oxford University Press.

Asendorpf, J. B., \& Motti-Stefanidi, F. (2018). Mediated disposition - environment transactions: The DAE model. European Journal of Personality, 32, 167-185.

Asendorpf, J. B., \& Rauthmann, J. F. (2020,). States and situations, traits and environments. In P. J. Corr \& G. Matthews (Eds.), Cambridge Handbook of Personality Psychology. Cambridge University Press.

Asendorpf, J. B., \& van Aken, M. A. G. (2003). Personality-relationship transaction in adolescence: Core versus surface personality characteristics. Journal of Personality, 71, 629-666.

Asendorpf, J. B., \& Wilpers, S. (1998). Personality effects on social relationships. Journal of Personality and Social Psychology, 74, 1531-1544. 
Atherton, O. E., Tackett, J. L., Ferrer, E., \& Robins, R. W. (2017). Bidirectional pathways between relational aggression and temperament from late childhood to adolescence. Journal of Research in Personality, 67, 75-84.

Atherton, O. E., Zheng, L. R., Bleidorn, W., \& Robins, R. W. (2019). The co-development of effortful control and school behavioral problems. Journal of Personality and Social Psychology, 117, 659-673.

Back, M. D., Baumert, A., Denissen, J. J. A., Hartung, F.-M., Penke, L., Schmukle, S. C., Schönbrodt, F. D., Schröder-Abé, M., Vollmann, M., Wagner, J., \& Wrzus, C. (2011). PERSOC: A unified framework for understanding the dynamic interplay of personality and social relationships. European Journal of Personality, 25, 90-107.

Bahns, A. J., Crandall, C. S., Gillath, O., \& Preacher, K. J. (2017). Similarity in relationships as niche construction: Choice, stability, and influence within dyads in a free choice environment. Journal of Personality and Social Psychology, 112, 329-355.

Balliet, D., Tybur, J. M., \& Van Lange, P. A. (2017). Functional interdependence theory: An evolutionary account of social situations. Personality and Social Psychology Review, 21, 361-388.

Baltes, P. B. (1987). Theoretical propositions of life-span developmental psychology: On the dynamics between growth and decline. Developmental Psychology, 23, 611-626.

Baltes, P. B. (1997). On the incomplete architecture of human ontogeny: Selection, optimization, and compensation as foundation of developmental theory. American Psychologist, 52, 366-380.

Baltes, P. B., \& Baltes, M. M. (1990). Psychological perspectives on successful aging: The model of selective optimization with compensation. In P. B. Baltes \& M. M. Baltes (Eds.), Successful aging: Perspectives from the behavioral sciences (pp. 1-34). Cambridge University Press.

Baltes, P. B., Lidenberger, U., \& Staudinger, U. M. (2006). Life span theory in developmental psychology. In W. Damon \& R. M. Lerner (Eds.). Handbook of child psychology, volume one: Theoretical models of human development ( $6^{\text {th }}$ ed., pp. 569-664). John Wiley \& Sons, Inc.

Baltes, P. B., Staudinger, U. M., \& Lindenberger, U. (1999). Lifespan psychology: Theory and application to intellectual functioning. Annual Review of Psychology, 50, 471-507. 
Bandura, A. (1978). The self system in reciprocal determinism. American Psychologist, 33, 344358.

Bandura, A. (1982). The psychology of chance encounters and life paths. American Psychologist, 37, 747-755.

Barranti, M., Carlson, E. N., \& Côté, S. (2017). How to test questions about similarity in personality and social psychology research: Description and empirical demonstration of response surface analysis. Social Psychological and Personality Science, 8, 465-475.

Baumeister, R. F., \& Leary, M. R. (1995). The need to belong: Desire for interpersonal attachments as a fundamental human motivation. Psychological Bulletin, 117, 497-529.

Baumert, A., Schmitt, M., Perugini, M., Johnson, W., Blum, G., Borkenau, P., Costantini, G., Denissen, J. J. A., Fleeson, W., Grafton, B., Jayawickreme, E., Kurzius, E., MacLeod, C., Miller, L. C., Read, S. J., Roberts, B., Robinson, M. D., Wood, D., \& Wrzus, C. (2017). Integrating personality structure, personality process, and personality development. European Journal of Personality, 31, 503-528.

Beck, E. D., \& Jackson, J. J. (2020). Idiographic traits: A return to Allportian approaches to personality. Current Directions in Psychological Science. https://doi.org/10.1177/0963721420915860

Bell, R. Q., \& Chapman, M. (1986). Child effects in studies using experimental or brief longitudinal approaches to socialization. Developmental Psychology, 22, 595-603.

Belsky, J., \& Pluess, M. (2009). Beyond diathesis stress: Differential susceptibility to environmental influences. Psychological Bulletin, 135, 885-908.

Biesanz, J. C. (2010). The social accuracy model of interpersonal perception: Assessing individual differences in perceptive and expressive accuracy. Multivariate Behavioral Research, 45, 853-885.

Biesanz, J. C., \& West, S. G. (2000). Personality coherence: Moderating self-other profile agreement and profile consensus. Journal of Personality and Social Psychology, 79, 425437.

Blake, A. B., Lee, D., De la Rosa, R., \& Sherman R. A. (in press). Wearable Cameras, Machine Vision, and Big Data Analytics: Insights into People and the Places they go. In Woo, S. E., Tay, L., \& Proctor, R. (Eds.), Big Data Methods for Psychological Research: New Horizons and Challenges. APA Books. 
Bleidorn, W., \& Denissen, J. J. A. (in press). Personality and life transitions in early adulthood. In O. P. John \& R. W. Robins (Eds.), Handbook of personality: Theory and research. Guilford.

Bleidorn, W., \& Hopwood, C. J. (2019). Using Machine Learning to Advance Personality Assessment and Theory. Personality and Social Psychology Review, 23, 190-203.

Bleidorn, W., Hopwood, C. J., \& Lucas, R. E. (2018). Life Events and Personality Change. Journal of Personality, 86, 83-96.

Bleidorn, W., Kandler, C., Hülsheger, U. R., Riemann, R., Angleitner, A., \& Spinath, F. M. (2010). Nature and nurture of the interplay between personality traits and major life goals. Journal of Personality and Social Psychology, 99, 366-379.

Bleidorn, W., Schönbrodt, F., Gebauer, J. E., Rentfrow, P. J., Potter, J., \& Gosling, S. D. (2016). To Live Among Like-Minded Others: Exploring the Links Between Person-City Personality Fit and Self-Esteem. Psychological Science, 27, 419-427.

Blum, G. S., Rauthmann, J. F., Göllner, R., Lischetzke, T., \& Schmitt, M. (2018). The Nonlinear Interaction of Person and Situation (NIPS) Model: Theory and Empirical Evidence. European Journal of Personality, 33, 286-305.

Blum, G. S., \& Schmitt, M. (2015). Towards a state-trait-model for situations. European Journal of Personality, 29, 386-388.

Blum, G. S., \& Schmitt, M. (2017). The nonlinear interaction of person and situation (NIPS) model and its values for a psychology of situations. In J. F. Rauthmann, R. A. Sherman, \& D. C. Funder (Eds.), Oxford Handbook of Psychological Situations. Oxford University Press.

Boele, S., Sijtsema, J. J., Klimstra, T. A., Denissen, J. J. A., and Meeus, W. H. J. (2017). PersonGroup Dissimilarity in Personality and Peer Victimization. European Journal of Personality, 31, 220-233.

Bolger, N., \& Schilling, E. A. (1991). Personality and problems of everyday life: the role of neuroticism in exposure and reactivity to daily stressors. Journal of Personality, 59, 355386.

Bond, M. H. (2013). A general model for explaining situational influence on individual social behavior: Refining Lewin's formula. Asian Journal of Social Psychology, 16, 1-15. 
Bouchard, T. J., Jr., Lykken, D. T., Tellegen, A., \& McGue, M. (1996). Genes, drives, environment, and experience: EPD theory revised. In C. Benbow \& D. J. Lubinski (Eds.), Intellectual talent: Psychometric and social issues (pp. 5-43). John Hopkins University Press.

Bowers, K. S. (1973). Situationism in psychology: An analysis and a critique. Psychological Review, 80, 307-336.

Bowlby, J. (1973). Attachment and loss: Vol. II. Separation: Anxiety and anger. Basic Books.

Box, G. P., \& Draper, N. R. (1987). Empirical model-building and response surfaces. John Wiley \& Sons.

Boyle, G. J. (2019). Overarching personality paradigm: A neo-Cattellian psychometric model. Personality and Individual Differences, 147, 317-325.

Breil, S. M., Geukes, K., Wilson, R. E., Nestler, S., Vazire, S., \& Back, M. D. (2019). Zooming into real-life extraversion-How personality and situation shape sociability in social interactions. Collabra Psychology, 5(1), Article 7.

Briley, D. A. (2020). Behavioral genetic approaches for situation research. In J. F. Rauthmann, R. A. Sherman, \& D. C. Funder (Eds.), The Oxford Handbook of Psychological Situations. Oxford University Press.

Briley, D. A., Livengood, J., Derringer, J., Tucker-Drob, E. M., Fraley, R. C., \& Roberts, B. W. (2019). Interpreting behavior genetic models: Seven developmental processes to understand. Behavior Genetics, 49, 196-210.

Briley, D. A., \& Tucker-Drob, E. M. (2014). Genetic and environmental continuity in personality development: A meta-analysis. Psychological Bulletin, 140, 1303-1331.

Brown, N. A., Blake, A. B., \& Sherman, R. A. (2017). A snapshot of the life as lived: Wearable cameras in social and personality psychological science. Social Psychological and Personality Science, 8, 592-600.

Buss, D. M. (1987). Selection, evocation, and manipulation. Journal of Personality and Social Psychology, 53, 1214-1221.

Buss, D. M. (1991). Evolutionary personality psychology. Annual Review of Psychology, 42, 459-491.

Buss, D. M., \& Greiling, H. (1999). Adaptive individual differences. Journal of Personality, 67, 209-243. 
Cable, D. M., \& Edwards, J. R. (2004). Complementary and supplementary fit: A theoretical and empirical integration. Journal of Applied Psychology, 89, 822-834.

Cable, D. M., \& Judge, T. A. (1996). Person-organization fit, job choice decisions, and organizational entry. Organizational Behavior \& Human Decision Processes, 67, 294311.

Caldwell, D. F., \& O’Reilly, C. A. (1990). Measuring person-job fit with a profile comparison process. Journal of Applied Psychology, 75, 648-657.

Camperio Ciani, A. S., Capiluppi, C., Veronese, A., \& Sartori, G. (2007). The adaptive value of personality differences revealed by small island population dynamics. European Journal of Personality, 21, 3-22.

Caplan, R. D. (1987). Person-environment fit theory and organizations: Commensurate dimensions, time perspectives, and mechanisms. Journal of Vocational Behavior, 31, 248267.

Caspi, A., \& Bem, D. J. (1990). Personality continuity and change across the life course. In L. Pervin (Ed.), Handbook of personality: Theory and research ( $1^{\text {st }}$ ed., pp. 549-575). Guilford Press.

Caspi, A., Bem, D. J., \& Elder, G. H., Jr. (1989). Continuities and consequences of interactional styles across the life course. Journal of Personality, 57, 375-406.

Caspi, A., McClay, J., Moffitt, T., Mill, J., Martin, J., Craig, I. W., Taylor, A., \& Poulton, R. (2002). Role of genotype in the cycle of violence in maltreated children. Science, 297, 851-854.

Caspi, A., \& Moffitt, T. E. (1991). Individual differences are accentuated during periods of social change: The sample case of girls at puberty. Journal of Personality and Social Psychology, 61, 157-168.

Caspi, A. \& Moffitt, T. E. (1993). When do individual differences matter? A paradoxical theory of personality coherence. Psychological Inquiry, 4, 247-271.

Caspi, A., \& Roberts, B. W. (1999). Personality continuity and change across the life course. In L. A. Pervin \& O. P. John (Eds.), Handbook of personality: Theory and research (Vol. 2, pp. 300-326). Guilford Press.

Caspi, A., \& Roberts, B. W. (2001). Personality development across the life span: The argument for change and continuity. Psychological Inquiry, 12, 49-66. 
Caspi, A., Roberts, B. W., \& Shiner, R. L. (2005). Personality Development: Stability and Change. Annual Review of Psychology, 56, 453-484.

Cattell, R. B., Cattell, A, K. S., \& Rhymer, R. M. (1947). P-technique demonstrated in determining psycho-physiological source traits in a normal individual. Psychometrika, 12, 267-288.

Cesario, J., Grant, H., \& Higgins, E. T. (2004). Regulatory fit and persuasion: Transfer from “feeling right". Journal of Personality and Social Psychology, 86, 388-404.

Chatman, J. (1991). Matching people and organizations: Selection and socialization in public accounting firms. Administrative Science Quarterly, 36, 459-484.

Chemero, A. (2001). What we perceive when we perceive affordances. Ecological Psychology, 13, 111-116.

Chemero, A. (2003). An outline of a theory of affordances. Ecological Psychology, 15, 181-195.

Chopik, W. J., \& Motyl, M. (2016). Ideological Fit Enhances Interpersonal Orientations. Social Psychological and Personality Science, 7, 759-768.

Ciani, A. C., \& Capiluppi, C. (2011). Gene flow by selective emigration as a possible cause for personality differences between small islands and mainland populations. European Journal of Personality, 25, 53-64.

Clark, M. S., Lemay, E. P., Jr., \& Reis, H. T. (2020). Other People as Situations: Relational Context Shapes Psychological Phenomena. In J. F. Rauthmann, R. A. Sherman, \& D. C. Funder (Eds.), The Oxford Handbook of Psychological Situations. Oxford University Press.

Cole, D. A., \& Maxwell, S. E. (2003). Testing mediational models with longitudinal data: Questions and tips in the use of structural equation modeling. Journal of Abnormal Psychology, 112, 558-577.

Cooper, A. B., Sherman, R. A., Rauthmann, J. F., Serfass, D. G., \& Brown, N. A. (2018). Feeling good and authentic: Experienced authenticity in daily life is predicted by positive feelings and situation characteristics, not trait-state consistency. Journal of Research in Personality, 77, 57-69.

Cooper, C. (1974). The house as symbol of the self. In J. Lang, C. Burnette, W. Moleski, \& D. Vachon (Eds.), Designing/or human behavior (pp. 130-146). Dowden, Hutchinson, \& Ross. 
Cooper, W. H., \& Withey, M. J. (2009). The strong situation hypothesis. Personality and Social Psychology Review, 13, 62-72.

Corker, K. S., \& Donnellan, M. B. (2020). Person-situation transactions across the life span. In J. F. Rauthmann, R. A. Sherman, \& D. C. Funder (Eds.), The Oxford Handbook of Psychological Situations. Oxford University Press.

Costa, P. T., Jr., Herbst, J. H., McCrae, R. R., \& Siegler, I. C. (2000). Personality at midlife: Stability, intrinsic maturation, and response to life events. Assessment, 7, 365-378.

Costa, P. T., Jr., McCrae, R. R., \& Löckenhoff, C. E. (2019). Personality across the life span. Annual Review of Psychology, 70, 423-448.

Costantini, G., \& Perugini, M. (2018). A framework for testing causality in personality research. European Journal of Personality, 32, 254-268.

Craik, K. H. (1994). Manifestations of individual differences in personality within everyday environments. In D. Bartussek \& M. Amelang (Eds.), Fortschritte der Differentiellen Psychologie und Psychologischen Diagnostik: Festschrift zum 60. Geburtstag von Professor Dr. Kurt Pawlik [Advances in differential psychology and psychological assessment: Festschrift on the occasion of the $60^{\text {th }}$ birthday of Professor Dr. Kurt Pawlik] (pp. 19 -25). Hogrefe.

Cramer, A. O. J., Van der Sluis, S., Noordhof, A., Wichers, M., Geschwind, N., Aggen, S. H., Kendler, K. S., \& Borsboom, D. (2012). Dimensions of normal personality as networks in search of equilibrium: You can't like parties if you don't like people. European Journal of Personality, 26, 414-431.

Craik, K. H. (2000). The lived day of an individual: A person-environment perspective. In W. B. Walsh, K. H. Craik, \& R. H. Price (Eds.), Person-environment psychology: New directions and perspectives (pp.233-266). Erlbaum.

Cronbach, L. J. (1955). Process affecting scores on "understanding of others" and "assumed similarity". Psychological Bulletin, 52, 177-193.

Cronbach, L. J., \& Gleser, G. C. (1953). Assessing similarity between profiles. Psychological Bulletin, 50, 456-473.

Cronbach, L. J., \& Gleser, G. C. (1957). Psychological tests and personnel decisions. University of Illinois Press. 
Dalal, R. S., Alaybek, B., Sheng, Z., Holland, S. J., \& Tomassetti, A. J. (2019). Extending Situational Strength Theory to Account for Situation-Outcome Mismatch. Journal of Business and Psychology, 1-24.

Dalal, R. S., Meyer, R. D., Bradshaw, R. P., Green, J. P., Kelly, E. D., \& Zhu, M. (2015). Personality strength and situational influences on behavior: A conceptual review and research agenda. Journal of Management, 41, 261-287.

Day, R. L., Laland, K. N., \& Odling-Smee, F. J. (2003). Rethinking adaptation: the nicheconstruction perspective. Perspectives in Biology and Medicine, 46, 80-95.

De Fruyt, F., BartelsS, M., Van Leeuwen, K., De Clercq, B., Decuyper, M., \& Mervielde, I. (2006). Five types of personality continuity in childhood and adolescence. Journal of Personality and Social Psychology, 91, 538-552.

Denissen, J. J. A., Bleidorn, W., Hennecke, M., Luhmann, M., Orth, U., Specht, J., \& Zimmermann, J. (2018). Uncovering the Power of Personality to Shape Income. Psychological Science, 29, 3-13.

Denissen, J. J. A., Luhmann, M., Chung, J. M., \& Bleidorn, W. (2019). Transactions between life events and personality traits across the adult lifespan. Journal of Personality and Social Psychology, 116, 612-633.

Denissen, J. J. A., \& Penke, L. (2008). Individual reaction norms underlying the Five Factor Model of personality: First steps towards a theory-based conceptual framework. Journal of Research in Personality, 42, 1285-1302.

Denissen, J. J. A., Ulferts, H., Lüdtke, O., Muck, P. M., \& Gerstorf, D. (2014). Longitudinal transactions between personality and occupational roles: A large and heterogeneous study of job beginners, stayers, and changers. Developmental Psychology, 50, 1931-1942.

Dermody, S. S., Thomas, K. M., Hopwood, C. J., Durbin, C. E., \& Wright, A. G. C. (2017). Modeling the complexity of dynamic, momentary interpersonal behavior: Applying the time-varying effect model to test predictions from interpersonal theory. Journal of Research in Personality, 68, 54-62.

Deventer, J., Wagner, J., Lüdtke, O., \& Trautwein, U. (2019). Are personality traits and relationship characteristics reciprocally related? Longitudinal analyses of codevelopment in the transition out of high school and beyond. Journal of Personality and Social Psychology, 116, 331-347. 
DeYoung, C. G. (2015). Cybernetic Big Five Theory. Journal of Research in Personality, 56, $33-$ 58.

Diekman, A. B., Steinberg, M., Brown, E. R., Belanger, A. L., \& Clark, E. K. (2017). A goal congruity model of role entry, engagement, and exit: Understanding communal goal processes in STEM gender gaps. Personality and Social Psychology Review, 21, 142-175.

Diener, E., Larsen, R. J., \& Emmons, R. A. (1984). Person $\times$ Situation interactions: Choice of situations and congruence response models. Journal of Personality and Social Psychology, 47, 580-592.

Dodge, K. A. (1986). A social information-processing model of social competence in children. In M. Perlmutter (Ed.), Minnesota Symposium on Child Psychology (pp. 77-125). Erlbaum.

Duckworth, A. L., Gendler, T. S., \& Gross, J. J. (2016). Situational strategies for self-control. Perspectives on Psychological Science, 11, 35-55.

Dumont, F., \& Carson, A. D. (1995). Precursors of vocational psychology in ancient civilizations. Journal of Counseling \& Development, 73, 371-378.

Edwards, J. R. (2001). Ten difference score myths. Organizational Research Methods, 4, 265287.

Edwards, J. R. (2002). Alternatives to difference scores: Polynomial regression analysis and response surface methodology. In F. Drasgow \& N. W. Schmitt (Eds.), Advances in measurement and data analysis (pp. 350-400). Jossey-Bass.

Edwards, J. R. (2008). Person-environment fit in organizations: An assessment of theoretical progress. The Academy of Management Annals, 2, 167-230.

Edwards, J. R., Cable, D. M., Williamson, I. O., Lambert, L. S., \& Shipp, A. J. (2006). The phenomenology of fit: linking the person and environment to the subjective experience of person-environment fit. Journal of Applied Psychology, 91, 802-827.

Edwards, J. R., Caplan, R. D., \& Harrison, R. V. (1998). Person-environment fit theory: Conceptual foundations, empirical evidence, and directions for future research. In C. L. Cooper (Ed.), Theories of organizational stress (pp. 28-67). Oxford University Press.

Edwards, J. R., \& Cooper, C. L. (1990). The person-environment fit approach to stress: Recurring problems and some suggested solutions. Journal of Organizational Behavior, 11, 293307. 
Edwards, J. R., \& Parry, M. E. (1993). On the use of polynomial regression equations as an alternative to difference scores in organizational research. Academy of Management Journal, 36, 1577-1613.

Edwards, J. R., \& Rothbard, N. P. (2000). Mechanisms linking work and family: Clarifying the relationship between work and family constructs. The Academy of Management Review, 25, 178-199.

Edwards, J. R., \& Shipp. A. J. (2007). The relationship between person-environment fit and outcomes: An integrative theoretical framework. In C. Ostroff \& T. A. Judge (Eds.), Perspectives on organizational fit (pp. 209-258). Jossey-Bass.

Elder, G. H., Jr., \& Shanahan, M. J. (2006). The life course and human development. In W. Damon \& R. M. Lerner (Eds.). Handbook of child psychology, Volume one: Theoretical models of human development (6 ${ }^{\text {th }}$ ed., pp. 665-715). John Wiley \& Sons, Inc.

Ellis, B. J., Boyce, W. T., Belsky, J., Bakermans-Kranenburg, M. J., \& Van IJzendoorn, M. H. (2011). Differential susceptibility to the environment: An evolutionaryneurodevelopmental theory. Development and psychopathology, 23, 7-28.

Emmons, R. A., \& Diener, E. (1986a). A goal-affect analysis of everyday situational choices. Journal of Research in Personality, 20, 309-326.

Emmons, R. A., \& Diener, E. (1986b). Situation selection as a moderator of response consistency and stability. Journal of Personality and Social Psychology, 51, 1013-1019.

Emmons, R. A., Diener, E., \& Larsen, R. J. (1985). Choice of situations and congruence models of interactionism. Personality and Individual Differences, 6, 693-702.

Emmons, R. A., Diener, E., \& Larsen, R. J. (1986). Choice and avoidance of everyday situations and affect congruence: Two models of reciprocal interactionism. Journal of Personality and Social Psychology, 51, 815-826.

Endler, N. S. (1975). A person-situation interaction model for anxiety. In C. D. Spielberger \& I. G. Sarason (Eds.), Stress and Anxiety (Vol. 1, pp. 145-164). Hemisphere.

Endler, N. S., \& Hunt, J. M. (1966). Sources of behavioral variance as measured by the S-R Inventory of Anxiousness. Psychological Bulletin, 65, 336-346.

Endler, N. S., \& Magnusson, D. (1976). Toward an interactional psychology of personality. Psychological Bulletin, 83, 956-974. 
2492 Ekehammar, B. (1974). Interactionism in personality from a historical perspective. Psychological Bulletin, 81, 1026-1048.

Erikson, E. H. (1968). Identity: Youth and crisis. Norton \& Co.

Finkel, S. E. (1995). Causal analysis with panel data. Sage.

Fleeson, W. (2001). Toward a structure- and process-integrated view of personality: Traits as density distributions of states. Journal of Personality and Social Psychology, 80, 10111027.

Fleeson, W. (2007). Situation-based contingencies underlying trait-content manifestation in behavior. Journal of Personality, 75, 825-861.

Fleeson, W. (2012). Perspectives on the person: Rapid growth and opportunities for integration. In K. Deaux \& M. Snyder (Eds.), The Oxford Handbook of Personality and Social Psychology (pp. 33-63). Oxford University Press.

Fleeson, W. (2017). The production mechanisms of traits: Reflections on two amazing decades. Journal of Research in Personality, 69, 4-12.

Fleeson, W., \& Gallagher, P. (2009). The implications of big-five standing for the distribution of trait manifestation in behavior: Fifteen experience-sampling studies and a meta-analysis. Journal of Personality and Social Psychology, 97, 1097-1114.

Fleeson, W., \& Jayawickreme, E. (2015). Whole trait theory. Journal of Research in Personality, $56,82-92$.

Fleeson, W., \& Law, M. K. (2015). Trait enactments as density distributions: The role of actors, situations, and observers in explaining stability and variability. Journal of Personality and Social Psychology, 109, 1090-1104.

Fleeson, W., \& Noftle, E. (2008a). The end of the person-situation debate: An emerging synthesis in the answer to the consistency question. Social and Personality Psychology Compass, 2, 1667-1684.

Fleeson, W., \& Noftle, E. E. (2008b). Where does personality have its influence? A supermatrix of consistency concepts. Journal of Personality, 76, 1355-1385.

Ford, D. H., \& Lerner, R. M. (1992). Developmental systems theory: An integrative approach. Sage Publications, Inc. 
2521 Ford, M. T., Matthews, R. A., Wooldridge, J. D., Mishra, V., Kakar, U. M., \& Strahan, S. R.

2522

2523

2524

2525

2526

2527

2528

2529

2530

2531

2532

2533

2534

2535

2536

2537

2538

2539

2540

2541

2542

2543

2544

2545

2546

2547

2548

2549

2550

2551 (2014). How do occupational stressor-strain effects vary with time? A review and metaanalysis of the relevance of time lags in longitudinal studies. Work \& Stress, 28, 9-30.

Fournier, M. A., Moskowitz, D. S., \& Zuroff, D. C. (2008). Integrating dispositions, signatures, and the interpersonal domain. Journal of Personality and Social Psychology, 94, 531-545.

Fournier, M. A., Moskowitz, D. S., \& Zuroff, D. C. (2009). The interpersonal signature. Journal of Research in Personality, 43, 155-162.

Fraley, C., \& Roberts, B. W. (2005). Patterns of continuity: A dynamic model for conceptualizing the stability of individual differences in psychological constructs across the life course. Psychological Review, 112, 60-74.

French, J. R. P., Jr., Rodgers, W., \& Cobb, S. (1974). Adjustment as person-environment fit. In G. V. Coelho, D. A. Hamburg, \& J. E. Adams (Eds.), Coping and adaption (pp. 316-333). Basic Books.

Freund, A. M., \& Baltes, P. B. (1998). Selection, optimization, and compensation as strategies of life management: correlations with subjective indicators of successful aging. Psychology and Aging, 13, 531-543.

Freund, A. M., \& Baltes, P. B. (2000). The orchestration of selection, optimization, and compensation: An action-theoretical conceptualization of a theory of developmental regulation. In W. J. Perrig \& A. Grob (Eds.), Control of human behaviour, mental processes and consciousness (pp. 35-58). Erlbaum.

Freund, A. M., \& Baltes, P. B. (2002). Life-management strategies of selection, optimization and compensation: Measurement by self-report and construct validity. Journal of Personality and Social Psychology, 82, 642-662.

Fried, E. I. (2017) What are psychological constructs? On the nature and statistical modelling of emotions, intelligence, personality traits and mental disorders, Health Psychology Review, $11,130-134$.

Funder, D. C. (1995). On the accuracy of personality judgment: A realistic approach. Psychological Review, 102, 652-670.

Funder, D. C. (1999). Personality judgment: A realistic approach to person perception. Academic Press.

Funder, D. C. (2001). Personality. Annual Review of Psychology, 52, 197-221. 
2552 Funder, D. C. (2006). Towards a resolution of the personality triad: Persons, situations, and behaviors. Journal of Research in Personality, 40, 21-34.

Funder, D. C. (2008). Persons, situations and person-situation interactions. In O. P. John, R. Robins, \& L. A. Pervin (Eds.), Handbook of Personality (3 ${ }^{\text {rd }}$ ed., pp. 568-580). Guilford.

Funder, D. C. (2009). Persons, behaviors and situations: An agenda for personality psychology in the postwar era. Journal of Research in Personality, 43, 120-126.

Funder, D. C., \& Ozer, D. J. (2019). Evaluating Effect Size in Psychological Research: Sense and Nonsense. Advances in Methods and Practices in Psychological Science, 2, 156-168.

Furnham, A. (1981). Personality and activity preference. British Journal of Social Psychology, 20, 57-68.

Furr, R. M. (2008). A framework for profile similarity: Integrating similarity, normativeness, and distinctiveness. Journal of Personality, 76, 1267-1316.

Furr, R. M. (2010). The double-entry intraclass correlation as an index of profile similarity: Meaning, problems, and alternatives. Journal of Personality Assessment, 92, 1-15.

2566

Garretsen, H., Stoker, J. I., Soudis, D., Martin, R., \& Rentfrow, P. J. (2019). The relevance of personality traits for urban economic geography: Making space for psychological factors. Journal of Economic Geography, 19, 541-565.

Geiser, C., Götz, T., Preckel, F., \& Freund, P. A. (2017). States and traits: Theories, models, and assessment. European Journal of Psychological Assessment, 33, 219-223.

Geiser, C., Litson, K., Bishop, J., Keller, B. T., Burns, G. L., Servera, M., \& Shiffman, S. (2015). Analyzing person, situation, and person $\times$ situation interaction effects: Latent state-trait models for the combination of random and fixed situations. Psychological Methods, 20, 165-192.

Germain, C. B. (1981). The Ecological Approach to People-Environment Transactions. Social Casework, 62, 323-331.

Geukes, K., Nestler, S., Hutteman, R., Küfner, A. C. P., \& Back, M. D. (2017). Trait Personality and State Variability: Predicting Individual Differences in Within- and Cross-Context Fluctuations in Affect, Self-Evaluations, and Behavior in Everyday Life. Journal of Research in Personality, 69, 124-138.

Gibson, J. J. (1977). The theory of affordances. In R. Shaw \& J. Bransford (Eds.), Perceiving, Acting, and Knowing: Toward an Ecological Psychology (pp. 67-82). Lawrence Erlbaum. 
Gollob, H. F., \& Reichardt, C. S. (1987). Taking account of time lags in causal models. Child Development, 58, 80-92.

Gosling, S. D., Gaddis, S., \& Vazire, S. (2008). First impressions based on the environments we create and inhabit. In N. Ambady \& J. J. Skowronski (Eds.), First Impressions (pp. 334356). Guilford.

Gosling, S. D., Gifford, R., \& McCunn, L. (2013). The selection, creation, and perception of interior spaces: An environmental psychology approach. In G. Brooker \& L. Weinthal (Eds.), The Handbook of Interior Design (pp. 278-290). Berg.

Gosling, S. D., Ko, S. J., Mannarelli, T., \& Morris, M. E. (2002). A room with a cue: Personality judgments based on offices and bedrooms. Journal of Personality and Social Psychology, 82, 379-398.

Gifford, R. (2007). Environmental psychology: Principles and practice (4th ed.). Optimal Books. Gilbert, S. F., Bosch, T. C., \& Ledon-Rettig, C. (2015). Eco-Evo-Devo: Developmental symbiosis and developmental plasticity as evolutionary agents. Nature Reviews Genetics, $16,611-622$.

Graham, L. T., \& Sandy, C. J., \& Gosling, S. D. (2011). Manifestations of individual differences in physical and virtual environments. In T. Chamorro-Premuzic, S. von Stumm, \& A. Furnham (Eds.), Handbook of Individual Differences (pp. 773-800). Wiley-Blackwell.

Granger, C. W. J. (1969). Investigating causal relations by econometric models and cross-spectral methods. Econometrica, 37, 424-438.

Gross, J. J. (1998). The emerging field of emotion regulation: An integrative review. Review of General Psychology, 2, 271-299.

Haase, C., Heckhausen, J., \& Wrosch, C. (2013). Developmental regulation across the life span: Toward a new synthesis. Developmental Psychology, 49, 964-972.

Hamaker, E. L., Kuiper, R. M., \& Grasman, R. P. P. P. (2015). A critique of the cross-lagged panel model. Psychological Methods, 20, 102-116.

Hampson, S. E. (2012). Personality processes: Mechanisms by which personality traits "get outside the skin". Annual Review of Psychology, 63, 315-339.

Hankin, B. L., \& Abramson, L. Y. (2001). Development of gender differences in depression: An elaborated cognitive vulnerability-transactional stress theory. Psychological Bulletin, 127, 773-796. 
Harari, G. M., Müller, S. R., Aung, M. S. H., \& Rentfrow, P. J. (2017) Smartphone sensing methods for studying behavior in everyday life. Current Opinion in Behavioral Sciences, 18, 83-90.

Harari, G. M., Müller, S. R., Stachl, C., Wang, R., Wang, W., Bühner, M., Rentfrow, J. P., Campbell, A. T., \& Gosling, S. D. (in press). Sensing sociability: Individual differences in young adults' conversation, calling, texting and app use behaviors in daily life. Journal of Personality and Social Psychology. https://doi.org/10.1037/pspp0000245

Hardin, C. D., \& Higgins, E. T. (1996). Shared reality: How social verification makes the subjective objective. In E. T. Higgins \& R. M. Sorrentino (Eds.), Handbook of motivation and cognition: Vol. 3. The interpersonal context (pp. 28-84). Guilford.

Harms, P. D., Roberts, B. W., \& Winter, D. (2006). Becoming the Harvard man: Personenvironment fit, personality development, and academic success. Personality and Social Psychology Bulletin, 32, 851-865.

Harrison, D. A. (2007). Pitching fits in applied psychological research: Making fit methods fit theory. In C. Ostroff \& T. Judge (Eds.), Perspectives on organizational fit (pp. 389-416). Erlbaum.

Hedrick, P. W. (1990). Genotypic-specific habitat selection: A new model and its application. Heredity, 65, 145-149.

Heckhausen, J. (1997). Developmental regulation across adulthood: Primary and secondary control of age-related challenges. Developmental Psychology, 33, 176-187.

Hennecke, M., Bleidorn, W., Denissen, J. J. A., \& Wood, D. (2014). A three-part framework for self-regulated personality development across adulthood. European Journal of Personality, 28, 289-299.

Hertwig, R., \& Grüne-Yanoff, T. (2017). Nudging and boosting: Steering or empowering good decisions. Perspectives on Psychological Science, 12, 973-986.

Higgins, E. T. (2005). Value from regulatory fit. Current Directions in Psychological Science, 14, 209-213.

Hintz, F. A., Geiser, C., \& Shiffman, S. (2019). A latent state-trait model for analyzing states, traits, situations, method effects, and their interactions. Journal of Personality, 87, 434454. 
Hirschi, A. (2010). The role of chance events in the school-to-work transition: The influence of demographic, personality and career development variables. Journal of Vocational Behavior, 77, 39-49.

Hogan, J., \& Roberts, B. W. (1996). Issues and non-issues in the fidelity-bandwidth trade-off. Journal of Organizational Behavior, 7, 627-637.

Hogan, R. T. (1982). A socioanalytic theory of personality. In M. M. Page (Ed.), Nebraska Symposium on Motivation (pp. 55-89). University of Nebraska Press.

Hogan, R. T. (2009). Much ado about nothing: The person-situation debate. Journal of Research in Personality, 43, 249.

Hogan, R. T., \& Roberts, B. W. (2000). A Socioanalytic perspective on person/environment interaction. In W. B. Walsh, K. H. Craik, \& R. H. Price (Eds.), New directions in personenvironment psychology (pp. 1-24). Erlbaum.

Holland, J. L. (1959). A theory of vocational choice. Journal of Counseling Psychology, 6, 35-45.

Holland, J. L. (1966). The psychology of vocational choice. Blaisdell.

Holland, J. L. (1987). Some speculation about the investigation of person-environment transactions. Journal of Vocational Behavior, 31, 337-340.

Holland, J. L., \& Nichols, R. C. (1964). Explorations of a theory of vocational choice: III. A longitudinal study of change in major field of study. The Personnel and Guidance Journal, 43, 235-242.

Hollenstein, T., \& Lougheed, J. P. (2013). Beyond storm and stress: Typicality, transactions, timing, and temperament to account for adolescent change. American Psychologist, 68, 444-454.

Hong, R. Y., Chan, W. Y., \& Lim, J. Y. R. (2020). Pathological Personality Traits and the Experience of Daily Situations. Clinical Psychological Science, 8, 333-342.

Hopwood, C. J. (2018). Interpersonal Dynamics in Personality and Personality Disorders. European Journal of Personality, 32, 499-524.

Horstmann, K. T., Rauthmann, J. F., Sherman, R. A., \& Ziegler, M. (2020, in press). Unveiling an exclusive link: Predicting behavior with personality, situation perception, and affect in a pre-registered experience sampling study. Journal of Personality and Social Psychology. 
Horstmann, K. T., \& Ziegler, M. (2020, in press). Assessing Personality States: What to Consider when Constructing Personality State Measures. European Journal of Personality. https://doi.org/10.1002/per.2266.

Hudson, N. W., Roberts, B. W., \& Lodi-Smith, J. (2012). Personality trait development and social investment in work. Journal of Research in Personality, 46, 334-344.

Human, L. J., \& Biesanz, J. C. (2013). Targeting the good target: An integrative review of the characteristics and consequences of being accurately perceived. Personality and Social Psychology Review, 17, 248-272.

Human, L. J., Mignault, M.-C., Biesanz, J. C., \& Rogers, K. H. (2019). Why are well-adjusted people seen more accurately? The role of personality-behavior congruence in naturalistic social settings. Journal of Personality and Social Psychology, 117, 465-482.

Humberg, S., Nestler, S., \& Back, M. D. (2019). Response surface analysis in personality and social psychology: Checklist and clarifications for the case of congruence hypotheses. Social Psychological and Personality Science, 10, 409-419.

Ickes, W., Snyder, M., \& Garcia, S. (1997). Personality influences on the choice of situations. In R. Hogan, J. A. Johnson, \& S. R. Briggs (Eds.) Handbook of personality psychology (pp. 165-195). Academic Press.

Ilmarinen, V.-J., Vainikainen, M.-P., Verkasalo, M. J., \& Lönnqvist, J.-E. (2017). Homophilous friendship assortment based on personality traits and cognitive ability in middle childhood: The moderating effect of peer network size. European Journal of Personality, 31, 208-219.

Jayawickreme, E., \& Blackie, L. E. R. (2014). Posttraumatic growth as positive personality change: Evidence, controversies and future directions. European Journal of Personality, 28, 312-331.

Jayawickreme, E., \& Blackie, L. E. R. (2016). Exploring the Psychological Benefits of Hardship: A Critical Reassessment of Posttraumatic Growth. Springer.

Jayawickreme, E., Zachry, C. E., \& Fleeson, W. (2019). Whole trait theory: An integrative approach to examining personality structure and process. Personality and Individual Differences, 136, 2-11. 
Jeronimus, B. F., Riese, H., Sanderman, R., \& Ormel, J. (2014). Mutual reinforcement between neuroticism and life experiences: A five-wave, 16-year study to test reciprocal causation. Journal of Personality and Social Psychology, 107, 751-764.

Johnson, W. (2007). Genetic and environmental influences on behavior: Capturing all the interplay. Psychological Review, 114, 423-440.

Jokela, M. (2014). Flow of cognitive capital across rural and urban United States. Intelligence, $46,47-53$.

Jokela, M., Bleidorn, W., Lamb, M. E., Gosling, S. D., \& Rentfrow, P. J. (2015). Geographically varying associations between personality and life satisfaction in the London metropolitan area. Proceedings of the National Academy of Sciences, 112, 725-730.

Jokela, M., Elovainio, M., Kivimaki, M., \& Keltikangas-Jarvinen, L. (2008). Temperament and migration patterns in Finland. Psychological Science, 19, 831-837.

Jonkmann, K., Thoemmes, F., Lüdtke, O., \& Trautwein, U. (2014). Personality traits and living arrangements in young adulthood: Selection and socialization. Developmental Psychology, 50, 683-698.

Judd, C. M., Westfall, J., \& Kenny, D. A. (2012). Treating stimuli as a random factor in social psychology: A new and comprehensive solution to a pervasive but largely ignored problem. Journal of Personality and Social Psychology, 103, 54-69.

Judge, T. A., \& Zapata, C. P. (2015). The person-situation debate revisited: Effect of situation strength and trait activation on the validity of the big five personality traits in predicting job performance. Academy of Management Journal, 58, 1149-1170.

Kaiser, A., Bonsu, J. A., Charnigo, R. J., Milich, R., \& Lynam, D. R. (2016). Impulsive personality and alcohol use: Bidirectional relations over one year. Journal of Studies on Alcohol and Drugs, 77, 473-482.

Kandler, C., Bleidorn, W., Riemann, R., Angleitner, A., \& Spinath, F. M. (2012). Life events as environmental States and genetic traits and the role of personality: A longitudinal twin study. Behavior Genetics, 42, 57-72.

Kandler, C., Kornadt, A. E., Hagemeyer, B., \& Neyer, F. J. (2015). Patterns and sources of personality development in old age. Journal of Personality and Social Psychology, 109, 175-191. 
Keeler, K. R., Kong, W., Dalal, R. S., \& Cortina, J. M. (2019). Situational strength interactions: Are variance patterns consistent with the theory? Journal of Applied Psychology, 104, 1487-1513.

Kendal, J., Tehrani, J. J., \& Odling-Smee, J. (2011). Human niche construction in interdisciplinary focus. Philosophical Transactions of the Royal Society B: Biological Sciences, 366, 785-792.

Kendler, K. S., \& Baker, J. H. (2007). Genetic influences on measures of the environment: A systematic review. Psychological Medicine, 37, 615-626.

Kenny, D. A. (1994). Interpersonal Perception: A Social Relations Analysis. Guilford.

Kenrick, D. T., \& Funder, D. C. (1988). Profiting from controversy: Lessons from the personsituation debate. American Psychologist, 43, 23-34.

Kiff, C. J., Lengua, L. J., \& Zalewski, M. (2011). Nature and nurturing: Parenting in the context of child temperament. Clinical Child and Family Psychology Review, 14, 251-301.

Klimstra, T. A., Akse, J., Hale III, W. W., Raaijmakers, Q. A., \& Meeus, W. H. (2010). Longitudinal associations between personality traits and problem behavior symptoms in adolescence. Journal of Research in Personality, 44, 273-284.

Kosinski, M., Stillwell, D., \& Graepel, T. (2013). Private traits and attributes are predictable from digital records of human behavior. Proceedings of the National Academy of Sciences, 110, 5802-5805.

Kristof, A. L. (1996). Person-organization fit: An integrative review of its conceptualizations, measurement, and implications. Personnel Psychology, 49, 1-49.

Kristof-Brown, A. L., \& Guay, R. P. (2011). Person-environment fit. In S. Zedeck (Ed.), American Psychological Association handbook of industrial and organizational psychology (Vol. 3, pp. 3-50). American Psychological Association.

Krueger, J. I. (2009). A componential model of situation effects, person effects, and situation-byperson interaction effects on social behavior. Journal of Research in Personality, 43, 127 136.

Laceulle, O. M., \& van Aken, M. A. G. (2018). Transactions of personality and the social environment during development. In V. Zeigler-Hill \& T. K. Shackelford (Eds.), The SAGE handbook of personality and individual differences: Origins of personality and 
individual differences (pp. 241-259). Sage Reference. https://doi.org/10.4135/9781526451200.n14.

Laceulle, O. M., van Aken, M. A. G., Ormel, J., \& Nederhof, E. (2015). Stress-sensitivity and reciprocal associations between stressful events and adolescent temperament. Personality and Individual Differences, 81, 76-83.

Lakey, B. (2016). Understanding the $\mathrm{P} \times \mathrm{S}$ aspect of within-person variation: A variance partitioning approach. Frontiers in Psychology,7:2004. doi:10.3389/fpsyg.2015.02004.

Laland, K. N. (2004). Extending the extended phenotype. Biology and Philosophy, 19, 313-325.

Laland, K. N., \& Brown, G. R. (2006). Niche construction, human behavior, and the adaptive-lag hypothesis. Evolutionary Anthropology, 15, 95-104.

Laland, K. N., Matthews, B., \& Feldman, M. W. (2016). An introduction to niche construction theory. Evolutionary Ecology, 30, 191-202.

Laland, K. N., Odling-Smee, F. J., \& Feldman, M. W. (1996). The evolutionary consequences of niche construction: A theoretical investigation using two-locus theory. Journal of Evolutionary Biology, 9, 293-316.

Laland, K. N., Odling-Smee, F. J., \& Feldman, M. W. (1999). Evolutionary consequences of niche construction and their implications for ecology. Proceedings of the National Academy of Sciences, 96, 10242-10247.

Laland, K. N., Odling-Smee, J., \& Myles, S. (2010). How culture shaped the human genome: bringing genetics and the human sciences together. Nature Reviews Genetics, 11, 137148.

Laland, K. N., \& Sterelny, K. (2006). Perspective: Seven reasons (not) to neglect niche construction. Evolution, 60, 1751-1762.

Lang, F. R., Reschke, F. S., \& Neyer, F. J. (2006). Social relationships, transitions, and personality development across the life span. In D. K. Mroczek \& T. D. Little (Eds.), Handbook of personality development (pp. 445-466). Lawrence Erlbaum.

Law, M., Cooper, B., Strong, S., Stewart, D., Rigby, P., \& Letts, L. (1996). The PersonEnvironment-Occupation Model: A transactive approach to occupational performance. Canadian Journal of Occupational Therapy, 63, 9-23.

Leary, M. R. (1999). The social and psychological importance of self-esteem. In R. M. Kowalski \& M. R. Leary (Eds.), The social psychology of emotional and behavioral problems: 
Interfaces of social and clinical psychology (pp. 197-221). American Psychological Association.

Leary, M. R., \& Baumeister, R. F. (2000). The nature and function of self-esteem: Sociometer theory. In Advances in experimental social psychology (Vol. 32, pp. 1-62). Academic Press.

Leary, M. R., Tambor, E. S., Terdal, S. K., \& Downs, D. L. (1995). Self-esteem as an interpersonal monitor: The sociometer hypothesis. Journal of Personality and Social Psychology, 68, 518-530.

Lee, J. J. (2012). Correlation and causation in the study of personality. European Journal of Personality, 26, 372-390.

Leising, D., \& Müller-Plath, G. (2009). Person-situation integration in research on personality problems. Journal of Research in Personality, 43, 218-227.

Leising, D., Scharloth, J., Lohse, O., \& Wood, D. (2014). What types of terms do people use when describing an individual's personality? Psychological Science, 25, 1787-1794.

Lerner, R. M. (1978). Nature, nurture, and dynamic interactionism. Human Development, 21, 120.

Lerner, R. M., \& Walls, T. (1999). Revisiting Individuals as Producers of Their Development: From dynamic interactionism to developmental systems. In J. Brandtstädter \& R. M. Lerner (Eds.), Action \& self-development: Theory and research through the life span (pp. 3-36). Sage Publications, Inc.

Lewin, K. (1936). Principles of topological psychology. McGraw-Hill.

Lewin, K. (1943). Defining the "field at a given time." Psychological Review, 50, 292-310.

Lilienfeld, S. O, Sauvigné, K. C., Lynn, S. J., Cautin, R. L., Latzman, R. D., \& Waldman, I. D. (2015). Fifty psychological and psychiatric terms to avoid: A list of inaccurate, misleading, misused, ambiguous, and logically confused words and phrases. Frontiers in Psychology, 6:1100. doi:10.3389/fpsyg.2015.01100

Little, B. (1987). Personality and environment. In D. Stokols \& I. Altman (Eds.), Handbook of environmental psychology (Vol. 1, pp. 205-244). Wiley.

Lodi-Smith, J., \& Roberts, B. W. (2007). Social investment and personality: A meta-analysis of the relationship of personality traits to investment in work, family, religion, and volunteerism. Personality and Social Psychology Review, 11, 68-86. 
2825 Luhmann, M., Fassbender, I., Alcock, M., \& Haehner, P. (2020). A dimensional taxonomy of perceived characteristics of major life events. Journal of Personality and Social Psychology. Advance online publication. https://doi.org/10.1037/pspp0000291

Luhmann, M., Hofmann, W., Eid, M., \& Lucas, R. E. (2012). Subjective well-being and adaptation to life events: A meta-analysis. Journal of Personality and Social Psychology, 102, 592-615.

Luhmann, M., Orth, U., Specht, J., Kandler, C., \& Lucas, R. E. (2014). Studying changes in life circumstances and personality: It's about time. European Journal of Personality, 28, 256266.

Lüdtke, O., Roberts, B. W., Trautwein, U., \& Nagy, G. (2011). A random walk down university avenue: Life paths, life events, and personality trait change at the transition to university life. Journal of Personality and Social Psychology, 101, 620-637.

Luo, J., Derringer, J., Briley, D. A., \& Roberts, B. W. (2017). Genetic and environmental pathways underlying personality traits and perceived stress: Concurrent and longitudinal twin studies. European Journal of Personality, 31, 614-629.

Magnusson, D. (1990). Personality development from an interactional perspective. In L. A. Pervin (Ed.), Handbook of personality: Theory and research (pp. 193-222). Guilford.

Magnusson, D., \& Endler, N. S. (Eds.) (1977a). Personality at the crossroads: Current issues in interactional psychology. Erlbaum.

Magnusson, D., \& Endler, N. S. (1977b). Interactional psychology: Present status and future prospects. In D. Magnusson \& N. S. Endler (Eds.), Personality at the crossroads (pp. 335). Erlbaum.

Magnusson, D., \& Stattin, H. (2006). The person in context: A holistic-interactionist approach. In W. Damon \& R. M. Lerner (Eds.). Handbook of child psychology, volume one: Theoretical models of human development ( $6^{\text {th }}$ ed., pp. 400-464). John Wiley \& Sons, Inc. Mangelsdorf, J., Eid, M., \& Luhmann, M. (2019). Does growth require suffering? A systematic review and meta-analysis on genuine posttraumatic and postecstatic growth. Psychological Bulletin, 145, 302-338.

Manuck, S. B., \& McCaffery, J. M. (2014). Gene-environment interaction. Annual Review of Psychology, 65, 41-70. 
2855

2856

2857

2858

2859

2860

2861

2862

2863

2864

2865

2866

2867

2868

2869

2870

2871

2872

2873

2874

2875

2876

2877

2878

2879

2880

2881

2882

2883

2884

Markus, H. R., \& Kitayama, S. (2010). Cultures and selves: A cycle of mutual constitution. Perspectives on Psychological Science, 5, 420-430.

Marshall, M. A., \& Brown, J. D. (2006). Trait aggressiveness and situational provocation: A test of the traits as situational sensitivities (TASS) model. Personality and Social Psychology Bulletin, 32, 1100-1113.

McAdams, D. P., \& Pals, J. L. (2006). A new Big Five: Fundamental principles for an integrative science of personality. American Psychologist, 61, 204-217.

McCrae, R. R. (2019). Food for thought. In A. Realo (Ed.), In praise of an inquisitive mind: A festschrift in honor of Jüri Allik on the occasion of his $70^{\text {th }}$ birthday (pp. 57-66). University of Tartu Press.

McCrae, R. R. (2008). A note on some measures of profile agreement. Journal of Personality Assessment, 90, 105-109.

McCrae, R. R., \& Costa, P. T., Jr. (2008). The Five-Factor Theory of personality. In O. P. John, R. W. Robins, \& L. A. Pervin (Eds.), Handbook of personality: Theory and research $\left(3^{\text {rd }}\right.$ ed., pp. 159-181). Guilford.

Meagher, B. R. (2020). Ecologizing social psychology: The physical environment as a necessary constituent of social processes. Personality and Social Psychology Review, 24, 3-23.

Meglino, B. M., Ravlin, E. C., \& Adkins, C. L. (1992). The measurement of work value congruence: A field study comparison. Journal of Management, 18, 33-43.

Mehl, M. R., Gosling, S. D., \& Pennebaker, J. W. (2006). Personality in its natural habitat: Manifestations and implicit folk theories of personality in daily life. Journal of Personality and Social Psychology, 90, 862-877.

Meyer, R. D., \& Dalal, R. S. (2009). Situational strength as a means of conceptualizing context. Industrial and Organizational Psychology, 2, 99-102.

Meyer, R. D., Dalal, R. S., \& Hermida, R. (2010). A review and synthesis of situational strength in the organizational sciences. Journal of Management, 36, 121-140.

Meyer, R. D., Kelly, E. D., \& Bowling, N. A. (2020). Situational strength theory: A formalized conceptualization of a popular idea. In J. F. Rauthmann, R. A. Sherman, \& D. C. Funder (Eds.), The Oxford Handbook of Psychological Situations. Oxford University Press.

Miller, G. F. (2007). Reconciling evolutionary psychology and ecological psychology: How to perceive fitness affordances. Acta Psychologica Sinica, 39, 546-555. 
Miller, G. F. (2012). The smartphone psychology manifesto. Perspectives on Psychological Science, 7, 221-237.

Mischel, W. (1977). The interaction of person and situation. In D. Magnusson \& N. S. Endler (Eds.), Personality at the cross-rods: Current issues in interactional psychology (pp. 333352). Lawrence Erlbaum.

Mischel, W., \& Shoda, Y. (1995). A cognitive-affective system theory of personality: Reconceptualizing situations, dispositions, dynamics, and invariance in personality structure. Psychological Review, 102, 246-268.

Mischel, W., \& Shoda, Y. (1999). Integrating dispositions and processing dynamics within a unified theory of personality: The Cognitive Affective Personality System (CAPS). In L. A. Pervin \& O. P. John (Eds.), Handbook of Personality: Theory and Research (pp. 197218). Guilford.

Mischel, W., \& Shoda, Y. (2008). Toward a unified theory of personality: Integrating dispositions and processing dynamics within the Cognitive-Affective Processing System (CAPS). In O. P. John, R. W. Robins, \& L. A. Pervin (Eds.), Handbook of Personality (3 ${ }^{\text {rd }}$ Ee., pp. 208-241). Guilford.

Molho, C., \& Balliet, D. P. (2020). Navigating interdependent social situations. In J. F. Rauthmann, R. A. Sherman, \& D. C. Funder (Eds.), The Oxford Handbook of Psychological Situations. Oxford University Press.

Mortimer, J. T., \& Lorence, J. (1979). Work experience and occupational value socialization: A longitudinal study. American Journal of Sociology, 84, 1361-1385.

Mõttus, R. (2016). Towards more rigorous personality trait-outcome research. European Journal of Personality, 30, 292-303.

Mõttus, R., Allerhand, M., \& Johnson, W. (2017). Computational modeling of person-situation transactions: How accumulation of situational experiences can shape the distributions of trait scores. In J. F. Rauthmann, R. A. Sherman, \& D. C. Funder (Eds.), The Oxford Handbook of Psychological Situations. Oxford University Press.

Mõttus, R., \& Kandler, C. (2018). Special Issue: From Correlations to Explanations - Editorial: Causality is hard, but no cause for concern. European Journal of Personality, 32, 149324. 
Muchinsky, P. M., \& Monahan, C. J. (1987). What is person-environment congruence? Supplementary versus complementary models of fit. Journal of Vocational Behavior, 31, 268-277.

Mund, M., Finn, C., Hagemeyer, B., \& Neyer, F. J. (2016). Understanding dynamic transactions between personality traits and partner relationships. Current Directions in Psychological Science, 25, 411-416.

Mund, M. \& Nestler, S. (2019). Beyond the cross-lagged panel model: Next-generation statistical tools for analyzing interdependencies across the life course. Advances in Life Course Research, 41, 100249. https://doi.org/10.1016/j.alcr.2018.10.002

Mund, M., \& Neyer, F. J. (2014). Treating personality-relationship transactions with respect: Narrow facets, advanced models, and extended time frames. Journal of Personality and Social Psychology, 107, 352-368.

Murray, H. A. (1938). Explorations in personality. Oxford University Press.

Neyer, F. J., \& Asendorpf, J. B. (2001). Personality-relationship transaction in young adulthood. Journal of Personality and Social Psychology, 81, 1190-1204.

Neyer, F. J., \& Lehnart, J. (2007). Relationships matter in personality development: Evidence from an 8-year longitudinal study across young adulthood. Journal of Personality, 75, 535-568.

Neyer, F. J., Mund, M., Zimmermann, J., \& Wrzus, C. (2014). Personality-relationship transactions revisited. Journal of Personality, 82, 539-550.

Nestler, S., Humberg, S., \& Schönbrodt, F. D. (2019). Response surface analysis with multilevel data: Illustration for the case of congruence hypotheses. Psychological Methods, 24, 291308.

Nettle, D., \& Penke, L. (2010). Personality: Bridging the literatures from human psychology and behavioural ecology. Philosophical Transactions of the Royal Society B, 365, 4043-4050.

Nye, C. D., \& Roberts, B. W. (2019) A neo-socioanalytic model of personality development. In B. Baltes, C. Rudolph, \& H. Zacher (Eds.), Work Across the Lifespan (pp. 47-80). Elsevier.

Obschonka, M., Stützer, M., Rentfrow, P. J., Shaw-Taylor, L., Satchell, M., Silbereisen, R. K., Potter, J., \& Gosling, S. D. (2018). In the shadow of coal: How large-scale industries 
contributed to present-day regional differences in personality and well-being. Journal of Personality and Social Psychology, 115, 903-927.

Odling-Smee, F. J., Laland, K. N., \& Feldman, M. W. (2003). Niche construction: The neglected process in evolution. Princeton University Press.

Oishi, S. (2010). The Psychology of Residential Mobility: Implications for the Self, Social Relationships, and Well-Being. Perspectives on Psychological Science, 5, 5-21.

Olweus, D. (1977). A critical analysis of the modern interactionist position. In D. Magnusson \& N. S. Endler (Eds.), Personality at the Crossroads: Current Issues in Interactional Psychology (pp. 221-233). Wiley.

O’Reilly, C. A., Chatman, J., \& Caldwell, D. F. (1991). People and organizational culture: A profile comparison approach to assessing person-organization fit. Academy of Management Journal, 34, 487-516.

Orne, M. T. (1962). On the social psychology of the psychological experiment: With particular reference to demand characteristics and their implications. American Psychologist, 17, 776-783.

Parsons, F. (1909). Choosing a vocation. Houghton Mifflin.

Pearl, J. (2009). Causality: Models, reasoning, and inference (Vol. 2). Cambridge University Press.

Penke, L. (2010). Bridging the gap between modern evolutionary psychology and the study of individual differences. In D. M. Buss \& P. H. Hawley (Eds.), The evolution of personality and individual differences (pp. 243-279). Oxford University Press.

Penke, L., Denissen, J. J. A., \& Miller, G. F. (2007a). The evolutionary genetics of personality (target article). European Journal of Personality, 21, 549-587.

Penke, L., Denissen, J. J. A., \& Miller, G. F. (2007b). Evolution, genes, and inter-disciplinary personality research (authors' reply). European Journal of Personality, 21, 639-665.

Penke, L., \& Jokela, M. (2016). The evolutionary genetics of personality revisited. Current Opinion in Psychology, 7, 104-109.

Pérez-Edgar, K., MacNeill, L. A., \& Fu, X. (2020). Navigating through the experienced environment: insights from mobile eye tracking. Current Directions in Psychological Science. https://doi.org/10.1177/0963721420915880 
2976 2977

2978

2979

2980

2981

2982

2983

2984

2985

2986

2987

2988

2989

2990

2991

2992

2993

2994

2995

2996

2997

2998

2999

3000

3001

3002

3003

3004

3005

Pervin, L. A. (1968). Performance and satisfaction as a function of individual-environment fit. Psychological Bulletin, 69, 56-68.

Pettigrew, T. F. (1997). Personality and social structure: Social psychological contributions. In R. T. Hogan, J. A. Johnson, \& S. R. Briggs (Eds.), Handbook of personality psychology (pp. 417-438). Academic Press.

Pincus, A. L., Hopwood, C. J., \& Wright, A. G. C. (2017). The interpersonal situation: An integrative framework for the study of personality, psychopathology, and psychotherapy. In J. F. Rauthmann, R. A. Sherman, \& D. C. Funder (Eds.), The Oxford Handbook of Psychological Situations. Oxford University Press.

Plomin, R., DeFries, J. C., \& Loehlin, J. C. (1977). Genotype-environment interaction and correlation in the analysis of human behavior. Psychological Bulletin, 84, 309-322.

Plomin, R., DeFries, J. C., McClearn, G. E., \& McGuffin, P. (2008). Behavioral genetics (5 ${ }^{\text {th }}$ ed.). Freeman.

Plomin, R., Lichtenstein, P., Pedersen, N. L., McClearn, G. E., \& Nesselroade, J. R. (1990). Genetic influence on life events during the last half of the life span. Psychology and Aging, 5, 25-30.

Proshansky, H. M., Fabian, A. K., \& Kaminoff, R. (1983). Place identity: Physical world socialization of the self. Journal of Environmental Psychology, 3, 57-83.

Purcell, S. (2002). Variance component models for gene-environment interaction in twin analysis. Twin Research and Human Genetics, 5, 554-571.

Quirin, M., Robinson, M. D., Rauthmann, J. F., Kuhl, J., Read, S. J., Tops, M., \& DeYoung, C. (in revision). Uncovering the causal mechanisms of personality: The Personality Dynamics Approach. Manuscript currently under review.

Rauthmann, J. F. (2013). Effects of supplementary and complementary personality-situation fit on personality processes. Journal Psychologie des Alltagshandelns / Psychology of Everyday Activity, 6, 41-62.

Rauthmann, J. F. (2015a). Persönlichkeit als informationsprozessierendes System: Ein systemisch-synergetischer Zugang. [Personality as an information processing system: A systemic-synergetic approach.] Journal Psychologie des Alltagshandelns / Psychology of Everyday Activity, 8, 36-64. 
3006

3007

3008

3009

3010

3011

3012

3013

3014

3015

3016

3017

3018

3019

3020

3021

3022

3023

3024

3025

3026

3027

3028

3029

3030

3031

3032

3033

3034

3035

Rauthmann, J. F. (2015b). Structuring situational information: A road map of the multiple pathways to different situational taxonomies. European Psychologist, 20, 176-189.

Rauthmann, J. F. (2016). Motivational factors in the perception of psychological situation characteristics. Social and Personality Psychology Compass, 10, 92-108.

Rauthmann, J. F. (2017). Implicit personality development. In J. Specht (Ed.), Personality development across the lifespan (pp. 537-554). Elsevier.

Rauthmann, J. F. (2018a). Person-situation interactions. In B. J. Carducci (Ed.), The Wiley Encyclopedia of Personality and Individuals Differences (Volume Area II: Research Methods and Techniques of Assessment). John Wiley \& Sons, Inc.

Rauthmann, J. F. (2018b). Using situations as diagnostic agents for personality assessment. Invited commentary on a target article on "Personality-situation interplay in personnel selection" by Filip Lievens. European Journal of Personality, 31, 479-481.

Rauthmann, J. F. (2018c). Person-situation transactions can illuminate personality processes and dynamics. Invited commentary on a target article on "Personality dynamics and processes" by Anna Baumert et al. European Journal of Personality, 31, 562-564.

Rauthmann, J. F. (2020, May). Person-Environment Relations (Figures). Retrieved from osf.io/ds6mw

Rauthmann, J. F., Gallardo-Pujol, D., Guillaume, E. M., Todd, E., Nave, C. S., Sherman, R. A., Ziegler, M., Jones A. B., \& Funder, D. C. (2014). The Situational Eight DIAMONDS: A taxonomy of major dimensions of situation characteristics. Journal of Personality and Social Psychology, 107, 677-718.

Rauthmann, J. F., Horstmann, K. T., \& Sherman, R. A. (in prep.). Different data-analytical strategies to compute fit. Manuscript in preparation. See also osf.io/3ens5

Rauthmann, J. F., Horstmann, K. T., \& Sherman, R. A. (2020). The Psychological Characteristics of Situations: Towards an Integrated Taxonomy. In J. F. Rauthmann, R. A. Sherman, \& D. C. Funder (Eds.), The Oxford Handbook on Psychological Situations. Oxford University Press.

Rauthmann, J. F., Jones, A. B., \& Sherman, R. A. (2016). Directionality of person-situation transactions: Are there spillovers among and between situation experiences and personality states? Personality and Social Psychology Bulletin, 42, 893-909. 
3036 3037

3038

3039

3040

3041

3042

3043

3044

3045

3046

3047

3048

3049

3050

3051

3052

3053

3054

3055

3056

3057

3058

3059

3060

3061

3062

3063

3064

3065

3066

Rauthmann, J. F., \& Sherman, R. A. (2016). Situation change: Stability and change of situation variables between and within persons. Frontiers in Psychology, 6, 1938. doi:10.3389/fpsyg.2015.01938

Rauthmann, J., \& Sherman, R. (2019). Toward a research agenda for the study of situation perceptions: A variance componential framework. Personality and Social Psychology Review, 23, 238-266.

Rauthmann, J. F., \& Sherman, R. A. (2020, in press). The situation of situation research: Knowns and unknowns. Current Directions in Psychological Science.

Rauthmann, J. F., \& Sherman, R. A. (in prep.). Person-situation fit in daily life: Levels, stabilities, and correlates of trait-situation and state-situation fit. Manuscript in preparation. See also osf.io/t9sz6

Rauthmann, J. F., Sherman, R. A., \& Funder, D. C. (2015a). Principles of situation research: Towards a psychological understanding of situations (target article). European Journal of Personality, 29, 363-181.

Rauthmann, J. F., Sherman, R. A., \& Funder, D. C. (2015b). New horizons in research on psychological situations and environments (rejoinder to commentaries in response to the target article). European Journal of Personality, 29, 419-432.

Rauthmann, J. F., Sherman, R. A., \& Funder, D. C. (2020). The Oxford Handbook on Psychological Situations. Oxford University Press.

Rauthmann, J. F., Sherman, R. A., Nave, C. S., \& Funder, D. C. (2015c). Personality-driven situation experience, contact, and construal: How people's personality traits predict characteristics of their situations in daily life. Journal of Research in Personality, 55, 98111.

Read, S. J., Monroe, B. M., Brownstein, A. L., Yang, Y., Chopra, G., \& Miller, L. C. (2010). A neural network model of the structure and dynamics of human personality. Psychological Review, 117, 61-92.

Reis, H. T. (2008). Reinvigorating the Concept of Situation in Social Psychology. Personality and Social Psychology Review, 12, 311-329.

Reitz, A. K., Motti-Stefanidi, F., \& Asendorpf, J. B. (2016). Me, us, and them: Testing sociometer theory in a socially diverse real-life context. Journal of Personality and Social Psychology, 110, 908-920. 
Rentfrow, P. J. (Ed.) (2013). Geographical psychology: Exploring the interaction of environment and behavior. American Psychological Association.

Rentfrow, P. J., \& Gosling, S. D. (in press). Putting personality in its place: A geographical perspective on the distribution and expression of personality traits. In O. P. John and R. Robins, (Eds.) Handbook of Personality: Theory and Research. Guilford Press.

Rentfrow, P. J., Gosling, S. D., \& Potter, J. (2008). A Theory of the emergence, persistence, and expression of regional variation in basic traits. Perspectives on Psychological Science, 3, 339-369.

Rentfrow, P. J., \& Jokela, M. (2017). Regional differences in personality: Causes and consequences. In A. T. Church (Ed.), The Praeger handbook of personality across cultures: Trait psychology across cultures (pp. 225-249). Praeger/ABC-CLIO.

Rentfrow, P. J., \& Jokela, M. (2020). Geographical Variation in the Big Five Personality Domains. In D. Cohen \& S. Kitayama (Eds.) Handbook of Cultural Psychology $\left(2^{\text {nd }}\right.$ ed., pp. 768-792). Guilford Press.

Revelle, W. (1995). Personality processes. Annual Review of Psychology, 46, 295-328.

Richard, F. D., Bond, C. F., \& Stokes-Zoota, J. J. (2003). One Hundred Years of Social Psychology Quantitatively Described. Review of General Psychology, 7, 331-363.

Richardson, M. J., Dale, R., \& Marsh, K. L. (2014). Complex dynamical systems in social and personality psychology: Theory, modeling, and analysis. In H. T. Reis \& C. M. Judd (Eds.), Handbook of research methods in social and personality psychology (pp. 253282). Cambridge University Press.

Richards, J. M. (1993). Career development: A ten-year longitudinal study in population scientists. Journal of Career Assessment, 1, 181-192.

Riva, G., Baños, R. M., Botella, C., Mantovani, F., \& Gaggioli, A. (2016). Transforming experience: the potential of augmented reality and virtual reality for enhancing personal and clinical change. Frontiers in Psychiatry, 7, 164. https://doi.org/10.3389/fpsyt.2016.00164

Roberts, B. W. (2006). Personality development and organizational behavior. Research in Organizational Behavior, 27, 1-40.

Roberts, B. W. (2018). A Revised Sociogenomic Model of Personality Traits. Journal of Personality, 86, 23-35. 
Roberts, B. W., \& Caspi, A. (2003). The cumulative continuity model of personality development: Striking a balance between continuity and change in personality traits across the life course. In R. M. Staudinger \& U. Lindenberger (Eds.), Understanding Human Development: Lifespan Psychology in Exchange with Other Disciplines (pp. 183214). Kluwer Academic Publishers.

Roberts, B. W., Caspi, A., \& Moffitt, T. E. (2003). Work experiences and personality development in young adulthood. Journal of Personality and Social Psychology, 84, 582593.

Roberts, B. W., \& Damian, R. I. (2017). The principles of personality trait development and their relation to psychopathology. In D. Lynam \& D. Samuel (Eds.), Purdue symposium on psychological sciences. Oxford University Press.

Roberts, B. W., \& DelVecchio, W. F. (2000). The rank-order consistency of personality from childhood to old age: A quantitative review of longitudinal studies. Psychological Bulletin, 126, 3-25.

Roberts, B. W., Luo, J., Briley, D. A., Chow, P., Su, R., \& Hill, P. L. (2017). A systematic review of personality trait change through intervention. Psychological Bulletin, 143, 117-141.

Roberts, B. W., \& Nickel, L. B. (2017). A critical evaluation of the Neo-Socioanalytic Model of personality. In J. Specht (Ed.), Personality development across the lifespan (pp.157-178). Elsevier Academic Press.

Roberts, B. W., \& Nickel, L. B. (in press). Personality development across the life course: A Neo-socioanalytic perspective. In O. P. John \& R. W. Robins (Eds.), Handbook of Personality Theory and Research. Guilford Press.

Roberts, B. W., O’Donnell, M., \& Robins, R. W. (2004). Goal and personality trait development in emerging adulthood. Journal of Personality and Social Psychology, 87, 541-550.

Roberts, B. W., \& Pomerantz, E. M. (2004). On traits, situations, and their integration: A developmental perspective. Personality and Social Psychology Review, 8, 402-416.

Roberts, B. W., \& Robins, R. W. (2004). A longitudinal study of person-environment fit and personality development. Journal of Personality, 72, 89-110.

Roberts, B. W., Walton, K., \& Viechtbauer, W. (2006). Patterns of mean-level change in personality traits across the life course: A meta-analysis of longitudinal studies. Psychological Bulletin, 132, 1-25. 
Roberts, B. W., \& Wood, D. (2006). Personality development in the context of the NeoSocioanalytic Model of personality (Chapter 2, pp. 11-39). In D. Mroczek \& T. Little (Eds.), Handbook of Personality Development. Lawrence Erlbaum Associates.

Roberts, B. W., Wood, D., \& Lodi-Smith, J. (2005). Evaluating Five Factor Theory and social investment perspectives on personality trait development. Journal of Research in Personality, 39, 166-184.

Roisman, G. I., Newman, D. A., Fraley, R. C., Haltigan, J. D., Groh, A. M., \& Haydon, K. C. (2012). Distinguishing differential susceptibility from diathesis-stress: Recommendations for evaluating interaction effects. Development and Psychopathology, 24, 389-409.

Ross, L., \& Nisbett, R. E. (2011). The person and the situation: Perspectives of social psychology. Pinter \& Martin Publishers.

Rounds, J. B., Dawis, R. V., \& Lofquist, L. H. (1987). Measurement of person-environment fit and prediction of satisfaction in the theory of work adjustment. Journal of Vocational Behavior, 31, 297-318.

Rowe, D. C. (1987). Resolving the person-situation debate: Invitation to an interdisciplinary dialogue. American Psychologist, 42, 218-227.

Rubin, D. B. (1974). Estimating causal effects of treatments in randomized and nonrandomized studies. Journal of Educational Psychology, 66, 688-701.

Runyan, M. W. (1978). The life course as a theoretical orientation: Sequences of person-situation interaction. Journal of Personality, 46, 569-593.

Ryff, C. D. (1987). The place of personality and social structure research in social psychology. Journal of Personality and Social Psychology, 53, 1192-1202.

Sameroff, A. J. (1995). General systems theories and developmental psychopathology. In D. Cicchetti \& D. J. Cohen (Eds.), Developmental psychopathology (pp. 659-695). Wiley.

Saudino, K. J., Pedersen, N. L., Lichtenstein, P., McClearn, G. E., \& Plomin, R. (1997). Can personality explain genetic influences on life events? Journal of Personality and Social Psychology, 72, 196-206.

Scalco, M. D., Trucco, E. M., Coffman, D. L., \& Colder, C. R. (2015). Selection and socialization effects in early adolescent alcohol use: A propensity score analysis. Journal of Abnormal Child Psychology, 43, 1131-1143. 
Scarr, S. (1996). How people make their own environments: Implications for parents and policy makers. Psychology, Public Policy, and Law, 2, 204-228.

Scarr, S., \& McCartney, K. (1983). How people make their own environments: A theory of genotype $\rightarrow$ environment effects. Child Development, 54, 424-435.

Scheinerman, E. R. (1996). An invitation to dynamical systems. Prentice Hall.

Schmader, T., \& Sedikides, C. (2018). State authenticity as fit to environment: The implications of social identity for fit, authenticity, and self-segregation. Personality and Social Psychology Review, 22, 228-259.

Schmitt, M., \& Sabbagh, C. (2004). Synergistic person x situation interaction in distributive justice judgment and allocation behavior. Personality and Individual Differences, 37, 359371.

Schmitt, M., Eid, M., \& Maes, J. (2003). Synergistic person x situation interaction in distributive justice behavior. Personality and Social Psychology Bulletin, 29, 141-147.

Schneider, B. (2001). Fits about fit. Applied Psychology: An International Review, 50, 141-152.

Schneider, B., Goldstein, H. W., \& Smith, D. B. (1995). The ASA framework: An update. Personnel Psychology, 48, 747-773.

Schönbrodt, F. D. (2016a). RSA: An R package for response surface analysis (version 0.9.10). Retrieved from https://cran.r-project.org/package=RSA

Schönbrodt, F. D. (2016b). Testing fit patterns with polynomial regression models. Retrieved from osf.io/3889z

Scott-Phillips, T. C., Laland, K. N., Shuker, D. M., Dickins, T. E., \& West, S. A. (2014). The niche construction perspective: A critical appraisal. Evolution, 68, 1231-1243.

Saegert, S., \& Winkel, G. H. (1990). Environmental psychology. Annual Review of Psychology, 41, 441-477.

Shadish, W. R., Cook, T. D., \& Campbell, D. T. (2002). Experimental and quasi-experimental designs for generalized causal inference. Houghton, Mifflin and Company.

Sherman, R. A., \& Funder, D. C. (2009). Evaluating correlations in studies of personality and behavior: Beyond the number of significant findings to be expected by chance. Journal of Research in Personality, 43, 1053-1063. 
Sherman, R. A., Nave, C. S., \& Funder, D. C. (2012). Properties of persons and situations related to overall and distinctive personality-behavior congruence. Journal of Research in Personality, 46, 87-101.

Sherman, R. A., \& Pashler, H. (2019, May 24). Powerful Moderator Variables in Behavioral Science? Don't Bet on Them (Version 3). https://doi.org/10.31234/osf.io/c65wm

Sherman, R. A., Rauthmann, J. F., Brown, N., Serfass, D., \& Jones, B. (2015). The independent effects of personality and situations on real-time expressions of behavior and emotion. Journal of Personality and Social Psychology, 109, 872-888.

Shiner, R. L. (2009). The development of personality disorders: Perspectives from normal personality development in childhood and adolescence. Development and psychopathology, 21, 715-734.

Simons, D. J., Shoda, Y., \& Lindsay, D. S. (2017). Constraints on Generality (COG): A Proposed Addition to All Empirical Papers. Perspectives on Psychological Science, 12, 1123-1128.

Sliwinski, M. J. (2008). Measurement-burst designs for social health research. Social and Personality Psychology Compass, 2, 245-261.

Smith, R. E., Shoda, Y., Cumming, S. P., \& Smoll, F. L. (2009). Behavioral signatures at the ballpark: Intraindividual consistency of adults' situation-behavior patterns and their interpersonal consequences. Journal of Research in Personality, 43, 187-195.

Snow, R. (1989). Aptitude-Treatment Interaction as a framework for research on individual differences in learning. In P. Ackerman, R. J. Sternberg, \& R. Glaser (Eds.), Learning and Individual Differences (pp. 13-59). W. H. Freeman.

Snyder, M. (1981). On the influence of individuals on situations. In N. Cantor \& J. F. Kihlström (Eds.), Personality, cognition, and social interaction (pp. 309-329). Erlbaum.

Snyder, M. (1983). The influence of individuals on situations: Implications for understanding the links between personality and social behavior. Journal of Personality, 51, 497-516.

Snyder, M., \& Ickes, W. (1985). Personality and social behavior. In G. Lindzey \& E. Aronson (Eds.), Handbook of social psychology ( $3^{\text {rd }}$ ed., pp. 883-948). Random House.

Sosnowska, J., Kuppens, P., De Fruyt, F., \& Hofmans, J. (2019). A dynamic systems approach to personality: The Personality Dynamics (PersDyn) model. Personality and Individual Differences, 144, 11-18. 
Soto, C. J. (2019). How replicable are links between personality traits and consequential life outcomes? The Life Outcomes of Personality Replication Project. Psychological Science, 30, 711-727.

Spielberger, C. D. (1988). State-Trait Anger Expression Inventory. Research Edition. PAR.

Spirtes, P., Glymour, C., \& Scheines, R. (2001). Causation, prediction, and search (2 $2^{\text {nd }}$ ed.). MIT Press.

Spokane, A. R., Meir, E. I., \& Catalano, M. (2000). Person-environment congruence and Holland's theory: A review and reconsideration. Journal of Vocational Behavior, 57, 137187.

Srivastava, S., Angelo, K. M., \& Vallereux, S. R. (2008). Extraversion and positive affect: A day reconstruction study of person-environment transactions. Journal of Research in Personality, 42, 1613-1618.

Steyer, R., Ferring, D., \& Schmitt, M. J. (1992). States and Traits in Psychological Assessment. European Journal of Psychological Assessment, 8, 79-98.

Steyer, R., Mayer, A., Geiser, C., \& Cole, D. A. (2015). A theory of states and traits-Revised. Annual Review of Clinical Psychology, 11, 71-98.

Steyer, R., Schmitt, M., \& Eid, M. (1999). Latent State-Trait Theory and Research in Personality and Individual Differences. European Journal of Personality, 13, 389-408.

Stephenson, W. (1952). Some observations on Q Technique. Psychological Bulletin, 49, 483-498.

Stoffregen, T. A. (2000a). Affordances and events. Ecological Psychology, 12, 1-28.

Stoffregen, T. A. (2000b). Affordances and events: Theory and research. Ecological Psychology, 12, 93-107.

Stoffregen, T. A. (2003). Affordances as properties of the animal-environment system. Ecological Psychology, 15,115-134.

Stoffregen, T. A. (2004). Breadth and limits of the affordance concept. Ecological Psychology, $16,79-85$.

Stokols, D. (1995). The paradox of environmental psychology. American Psychologist, 50, 821837.

Stokols, D., Clitheroe, H. C., Jr., \& Zmuidzinas, M. (2000). Modeling and managing change in people-environment transactions. In W. B. Walsh, K. H. Craik, \& R. H. Price (Eds.), 
Person-environment psychology: New directions and perspectives (2nd ed., pp. 267-296). Lawrence Erlbaum Associates.

Stokols, D., \& Shumaker, S. A. (1981). People in places: A transactional view of settings. In J. H. Harvey (Ed.), Cognition, social behavior and the environment (pp. 441-488). Erlbaum.

Strong, S., Rigby, P., Stewart, D., Law, M., Letts, L., \& Cooper, B. (1999). Application of the Person-Environment-Occupation Model: A practical tool. Canadian Journal of Occupational Therapy, 66, 122-133.

Sturaro, C., Denissen, J. J. A., van Aken, M. A., \& Asendorpf, J. B. (2008). Person-environment transactions during emerging adulthood: The interplay between personality characteristics and social relationships. European Psychologist, 13, 1-11.

Sullivan, H. S. (1953). The interpersonal theory of psychiatry. Norton.

Sunstein, C. R., \& Reisch, L. A. (2017). The economics of nudge. Routledge.

Sutin, A. R., \& Costa, P. T. (2010). Reciprocal influences of personality and job characteristics across middle adulthood. Journal of Personality, 78, 257-288.

Tesser, A. (2002). Constructing a niche for the self: A bio-social, PDP-approach to understanding lives. Self and Identity, 1, 185-190.

Tett, R. P., Simonet, D. V., Walser, B., \& Cameron, B. (2013). Trait activation theory: Applications, further developments, and implications for workplace fit. In N. D. Christiansen \& R. P. Tett (Eds.), Handbook of personality at work (pp. 71-100). Routledge.

Thagard, P., \& Wood, J. V. (2015). Eighty phenomena about the self: Representation, evaluation, regulation, and change. Frontiers in Psychology, 6, 334. https://doi.org/10.3389/fpsyg.2015.00334

Thaler, R., \& Sunstein, C. (2008). Nudge. Penguin Books.

Thomas, A., \& Chess, S. (1977). Temperament and development. Brunner/Mazel.

Todd, P. M., \& Gigerenzer, G. (2020). The Ecological Rationality of Situations: Behavior = f(Adaptive Toolbox, Environment). In J. F. Rauthmann, R. A. Sherman, \& D. C. Funder (Eds.), The Oxford Handbook of Psychological Situations. Oxford University Press.

Turkheimer, E. (2000). Three laws of behavior genetics and what they mean. Current Directions in Psychological Science, 9, 160-164. 
Usami, S., Murayama, K., \& Hamaker, E. L. (2019). A unified framework of longitudinal models to examine reciprocal relations. Psychological Methods, 24, 637-657.

Vancouver, J. B., \& Schmitt, N. W. (1991). An exploratory examination of person-organization fit: Organizational goal congruence. Personnel Psychology, 44, 333-352.

Vazire, S. \& Gosling, S. D. (2004). e-perceptions: Personality impressions based on personal websites. Journal of Personality and Social Psychology, 87, 123-132.

Vazire, S., \& Sherman, R. A. (2017). Introduction to the special issue on within-person variability in personality (Editorial). Journal of Research in Personality, 69, 1-3.

Völkle, M. C., Gische, C., Driver, C. C., \& Lindenberger, U. (2018). The role of time in the quest for understanding psychological mechanisms. Multivariate behavioral research, 53, 782805.

Wachs, T. D. (1994). Fit, context, and the transition between temperament and personality. In C. F. Halverson Jr., G. A. Kohnstamm, \& R. P. Martin (Eds.), The developing structure of temperament and personality from infancy to adulthood (pp. 209-220). Erlbaum.

Wagerman, S. A., \& Funder, D. C. (2009). Situations. In P. J. Corr \& G. Matthews (Eds.), Cambridge Handbook of Personality Psychology (pp. 27-42). Cambridge University Press.

Wagner, J., Lüdtke, O., \& Voelkle, M. C. (2019). Using Dynamic Panel Models to Study Agerelated Differences and Time-related Changes in Personality. European Journal of Personality, 33, 420-434.

Walsh, W. B., Craik, K., \& Price, R. H. (2000). Person-environment psychology: New directions and perspectives ( $2^{\text {nd }}$ ed.). Lawrence Erlbaum Associates.

Wapner, S. (1987). A holistic, developmental, systems-oriented environmental psychology: Some beginnings. In D. Stokols \& I. Altman (Eds.), Handbook of environmental psychology (Vol. 2, pp. 1433-1465). Wiley.

Weidmann, R., Schönbrodt, F. D., Ledermann, T., \& Grob, A. (2017). Concurrent and longitudinal dyadic polynomial regression analyses of Big Five traits and relationship satisfaction: Does similarity matter? Journal of Research in Personality, 70, 6-15.

Wessels, N. M., Zimmermann, J., \& Leising, D. (2016). Toward a shared understanding of important consequences of personality. Review of General Psychology, 20, 426-436. 
Widaman, K. F., Helm, J. L., Castro-Schilo, L., Pluess, M., Stallings, M. C., \& Belsky, J. (2012). Distinguishing ordinal and disordinal interactions. Psychological Methods, 17, 615-622.

Wille, B., Beyers, W., \& De Fruyt, F. (2012). A transactional approach to person-environment fit: Reciprocal relations between personality development and career role growth across young to middle adulthood. Journal of Vocational Behavior, 81, 307-321.

Wilt, J., \& Revelle, W. (2015). Affect, Behaviour, Cognition and Desire in the Big Five: An Analysis of Item Content and Structure. European Journal of Personality, 29, 478-497.

Wood, D., \& Furr, R. M. (2016). The correlates of similarity estimates are often misleadingly positive: The nature and scope of the problem, and some solutions. Personality and Social Psychology Review, 20, 79-99.

Wood, D., \& Harms, P. D. (2016). Evidence of non-corresponsive causal relationships between personality traits and social power over time. Personality and Social Psychology Bulletin, $43,33-45$.

Wood, D., Lowman, G. H., Harms, P. D., \& Spain, S. M. (2019). Using functional fields to formally represent the meaning and logic of behavior: A worked example using Dark Triad-related actions. Personality and Individual Differences, 136, 24-37.

Wood, D., Spain, S. M., \& Harms, P. D. (2020). Functional approaches to representing the interplay of situations, persons, and behavior. In J. F. Rauthmann, R. A. Sherman, \& D. C. Funder (Eds.), The Oxford Handbook of Psychological Situations. Oxford University Press.

Woods, S. A., Lievens, F., De Fruyt, F., \& Wille, B. (2013). Personality across working life: The longitudinal and reciprocal influences of personality on work. Journal of Organizational Behavior, 34, S7-S25.

Wright, A. G. C., \& Hopwood, C. J. (2016). Advancing the assessment of dynamic psychological processes. Assessment, 23, 399-403.

Wright, A. G. C., \& Zimmermann, J. (2019). Applied ambulatory assessment: Integrating idiographic and nomothetic principles of measurement. Psychological Assessment, 31, 1467-1480.

Wrzesniewski, A. (2001). Crafting a job: Revisioning employees as active crafters of their work. Academy of Management Review, 26, 179-201. 
Wrzus, C., \& Mehl, M. (2015). Lab and/or field? Measuring personality processes and their social consequences. European Journal of Personality, 29, 250-271.

Wrzus, C., \& Neyer, F. J. (2016). Co-development of personality and friendships across the lifespan: An empirical review on selection and socialization. European Psychologist, 21, $254-273$.

Wrzus, C., \& Roberts, B. W. (2017). Processes of personality development in adulthood: The TESSERA framework. Personality and Social Psychology Review, 21, 253-277.

Wrzus, C., Wagner, G. G., \& Riediger, M. (2016). Personality-situation transactions from adolescence to old age. Journal of Personality and Social Psychology, 110, 782-799.

Yarkoni, T., \& Westfall, J. (2017). Choosing Prediction Over Explanation in Psychology: Lessons from Machine Learning. Perspectives on Psychological Science, 12, 1100-1122.

Youyou, W., Stillwell, D., Schwartz, H. A., \& Kosinski, M. (2017). Birds of a feather do flock together: Behavior-based personality-assessment method reveals personality similarity among couples and friends. Psychological Science, 28, 276-284.

Zaccaro, S. J., Green, J. P., Dubrow, S., \& Kolze, M. (2018). Leader individual differences, situational parameters, and leadership outcomes: A comprehensive review and integration. The Leadership Quarterly, 29, 2-43.

Zapko-Willmes, A., Riemann, R., \& Kandler, C. (2018). Unravelling quasi-causal environmental effects via phenotypic and genetically informed multi-rater models: The case of differential parenting and authoritarianism. European Journal of Personality, 32, 233253.

Zimmerman, J., \& Neyer, F. J. (2013). Do we become a different person when hitting the road? Personality development of sojourners. Journal of Personality and Social Psychology, $105,515-530$.

Zimmermann, J., Woods, W. C., Ritter, S., Happel, M., Masuhr, O., Jaeger, U., Spitzer, C., \& Wright, A. G. C. (2019). Integrating structure and dynamics in personality assessment: First steps toward the development and validation of a personality dynamics diary. Psychological Assessment, 31, 516-531.

Zuckerman, M. (1999). Diathesis-stress models. In M. Zuckerman (Ed.), Vulnerability to psychopathology: A biosocial model (pp. 2-23). American Psychological Association. 


\section{Box 1}

\section{Polynomial Regression and Response Surface Analysis}

The full second-degree polynomial regression in Equation 6 yields five regression weights ( $b_{1}$ : person variable; $b_{2}$ : environment variable; $b_{3}$ : square of the person variable; $b_{4}$ : person variable $\times$ environment variable interaction; $b_{5}$ : square of the environment variable) which can be used to compute the response surface parameters $a_{1-4}$. The latter define a three-dimensional response surface that is created when predicting an outcome variable on the $z$-axis by the full crossing of two predictor variables (e.g., a person and an environment variable), where one variable defines the $x$-axis and the other the $y$-axis. This is exemplified in Figure $4 d$ where the person, environment, and outcome variables are all measured on a standardized scale, ranging from -3 to +3 .

As can be seen, the response surface has a "Line of Congruence" (LOC; thick solid line) from $-3 /-3$ to $+3 /+3$ where values of the person and the environment variable, respectively, show exact numerical correspondence. The surface also has a "Line of Incongruence" (LOIC; thick dotted line) from $-3 /+3$ to $+3 /-3$ where the values of the person and the environment variable, respectively, show exact numerical noncorrespondence. Notably, neither the LOC nor the LOIC per se are the "fit" of the person and environment variable; "fit" is defined as an entire pattern of effects and thus more complex (Humberg et al., 2019; see 4.1.5 Outcome-based, Variable-oriented PersonEnvironment Fit). Both the LOC and LOIC have a linear slope ( $a_{1}$ and $a_{3}$, respectively) and a curvilinear slope ( $a_{2}$ and $a_{4}$, respectively). Applied to a scenario where an outcome variable is predicted from person and environment variables, the response surface parameters can be interpreted the following ways (for an accessible general summary, see Barranti, Carlson, \& Côté, 2017):

- The parameter $a_{1}\left(=b_{1}+b_{2}\right)$ concerns to what extent matches at higher values of the person and environment variable entail higher (or lower) values on an outcome variable than matches at lower values of the person and environment variable. The parameter will be strong when one or both of main effects of the person and/or the environment variable are present, potentially signaling additive effects. 
- The parameter $a_{2}\left(=b_{3}+b_{4}+b_{5}\right)$ concerns to what extent matches at more extreme values of the person and environment variable entail higher (or lower) values on an outcome variable than matches at less extreme values of the person and environment variable. The parameter will be strong when an interaction of the person with the environment variable and/or any non-linear effects of the person and the environment variables, respectively, are present.

- The parameter $a_{3}\left(=b_{1}-b_{2}\right)$ concerns to what extent one direction of mismatches in values (e.g., person variable values > environment variable values) entails higher (or lower) values on an outcome variable than the other direction of mismatches (e.g., environment variable values > person variable values). The parameter will be strong when either one of the main effects of the person and the environment variable, respectively, is (much) stronger than the other.

- The parameter $a_{4}\left(=b_{3}-b_{4}+b_{5}\right)$ concerns to what extent matches in values of person and environment variables entail higher (or lower) values on an outcome than mismatches.

Each response surface parameter allows a different insight into the data and together provide a more detailed picture on the effect pattern. It is also possible to construct confidence intervals around each parameter estimate and apply usual tests of statistical significance (if desired). While more parsimonious models with less predictors should be favored (and thus models with less predictors tested against models with more predictors), polynomial regressions may explain more variance and do consider quadratic effects which could be more common than expected (Edwards \& Parry, 1993). As can be seen in Figures 4a-c, response surfaces can be crafted to understand the relations between predictors and an outcome better even if not all predictors significantly or sizably predict the outcome. As such, they allow a more nuanced view on the data. Further, polynomial regressions are preferable to using difference scores (e.g., subtracting an environment score from a person score) as predictors of outcome variables because the latter possess several shortcomings despite having been used as indices of "fit" (Edwards, 2001, 2002). 


\section{Box 2}

\section{Analysis of Variable-oriented Person-Environment Correlations and Fits}

Estimating variable-oriented person-environment correlations or fits is straightforward: If there is one measurement occasion, then two concurrent person and environment variables are correlated, yielding a sample estimate of the correlation. This is usually all, but researchers may be interested in more. For example, additional questions could be: How strong are inter-individual differences in those correlations? Are those inter-individual differences stable across time (or age)? How do they develop or change? With which nomological variables (e.g., causes/antecedents, concomitants, consequences) are those inter-individual differences associated with? How strongly, when (moderations), and how (mediations) are they associated with those nomological variables? These and other questions can usually not be addressed when subscribing to a variable-oriented view, though they become readily answerable when using profile approaches (see 4.2.2 Person-oriented Profile Correlations).

However, there is a little-known work-around that allows estimating individual differences from a sample-level correlation. Asendorpf (1990) described such a procedure for obtaining individual consistency estimates (I) when correlating two scores across measurement occasions, but his procedure can be extended to any pair of correlated variables and thus also to variable-oriented person-environment correlations or fits. Specifically, for each individual in the sample, their individual correlation between any kind of commensurate person (PER) and environment variable (ENV), can be derived with following equation:

$$
I_{\text {PER.ENV }}=1-\frac{\left(z_{\mathrm{PER}}-z_{\mathrm{ENV}}\right)^{2}}{2}
$$

Equation 7

where $I_{\text {PER.ENV }}=$ individual person-environment correlation score, $z$ PER $=z$-score of an individual's score on the person variable PER, and $z \mathrm{ENV}=z$-score of an individual's score on the environment variable ENV. The resulting I IER.ENV scores will be heavily skewed. To use them for further analyses, a transformation (similar to Fisher's $r$-to- $z$ transformation) has to be applied to them first (see Asendorpf, 1990, p. 9 for exact details). It can be shown that the mean of all I PER.ENV scores represents the sample-level 
person-environment correlation. Deriving such $I_{\text {PER.ENV scores has so far not been done }}$ for person-environment relations research, but they can help expand the scope of variable-oriented analyses to examine the stability and nomological networks of interindividual differences in variable-oriented person-environment correlations or fits.

Another approach to estimating variable-oriented person-environment correlations or fits is available when there are several measurement occasions per person in the sample (e.g., as in experience sampling or longitudinal data). Then, hierarchical linear modeling or multi-level modeling (MLM) can be used to analyze such nested data (usually measurement occasions at level 1 nested within persons at level 2). In such models, one has to distinguish between a predictor (e.g., E: perceived level of Sociality in a given situation) and a criterion variable (e.g., P: perceived level of sociable behavior in a given situation). Although the designation as either predictor or criterion may seem arbitrary in non-lagged data where all associations are concurrent ${ }^{20}$, great care should be taken here and extracted slopes cautiously interpreted (as reversing predictors and outcomes could yield other conclusions). The level 1 associations between person and environment variables - here interpreted conceptually as person-environment correlations (although statistically in MLM they represent slopes) - have also been termed if-then patterns (Mischel \& Shoda, 1995, 1999, 2008), situation-behavior patterns (Smith et al., 2009), contingencies (Fleeson, 2007; Sherman et al., 2015), and signatures (Fournier et al., 2008, 2009). The advantage of using MLM is that the level 1 slopes can vary between individuals. If there are sizable inter-individual differences, these slopes may be associated, for example, across time with each other to examine their stability or with external correlates (such as personality traits, goals, well-being, etc.) to examine their nomological networks. In case of the latter, a level 2 predictor (e.g., a personality trait) is introduced that predicts the person-environment associations on level 1. Notably, such cross-level analyses then test interactions (see Sherman et al., 2015).

\footnotetext{
${ }^{20}$ If lagged data were used (for example, as in Rauthmann et al., 2016), then temporally clear predictoroutcome relations can be established. In such cases, person-environment transactions are examined. In crosssectional and non-lagged data, only concurrent associations can be modeled, and these are termed here conceptually person-environment correlations - even if statistically not a correlation coefficient, but slopes are estimated.
} 


\section{Box 3}

\section{Analysis of Profile-oriented Person-Environment Correlations and Fits}

In a profile approach, each person has a set of conceptually commensurate person and environment variables (Caplan, 1987) that are correlated. The profile correlation has been interpreted as person-environment fit, where the "gestalt," configuration, or pattern of the person and environment variables match up holistically with each other: one rankordered set of variables is correlated with the other rank-ordered set of variables within one person. ${ }^{21}$ The correlation (also termed in the literature as: association, agreement, concordance, correspondence, congruence, fit, matching, similarity) between two profiles can be quantified by $q$-correlations (Stephenson, 1952), which are mathematically regular Pearson product-moment correlations ranging from -1 (perfect negative correlation) to 0 (no systematic correlation) to +1 (perfect positive correlation). ${ }^{22}$ Notably, these correlations capture to what extent the rank-order among the person variables match the rank-order among the concordant environment variables. For example, if a person is

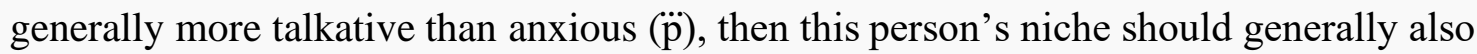
be more social than negative (ë). $Q$-correlations quantify how similar the shape of two profiles are (as the constituting profiles are standardized each to an elevation of $M=0$ and scatter of $S D=1$; Biesanz \& West, 2000; Furr, 2010). Although there are also other ways of quantifying the similarity between two profiles that take into account the elevation, scatter, and shape of the profiles to different degrees (e.g., $D^{2}, D^{\prime 2}$, and $D^{\prime \prime 2}$; Cronbach \& Gleser, 1953), $q$-correlations have been often favored because they (1)

\footnotetext{
${ }^{21}$ There are several methodological/statistical and conceptual complexities to this that cannot be elaborated on in detail here. Methodologically, different properties of the variables in the profiles (e.g., their number, positively and negatively keyed directions, variances, reliabilities etc.) as well as the mathematical estimation of the profile association index need to be considered. Conceptually, the point at which a profile association is termed "fit" also needs to be answered. For some, it is sufficient that the associated profiles are contentwise commensurate; any association is then a fit. However, one could also argue that "fit" only starts at a certain level of association (i.e., below that level we do not have conclusive evidence of "strong enough" fit and thus refrain from referring to fit there). Another approach would be to focus more on the conceptual content of the profiles. For example, the person and the environment profiles could "fit" together in a supplementary or a complementary way (see 4.3.1 Important Distinctions).

${ }^{22}$ Observed $q$-correlations can be tested in randomization procedures (e.g., Sherman \& Funder, 2009) against $q$-correlations obtained from random variable pairs (with the expectation that the latter are weaker than the former).
} 
produce findings consistent with other indices (e.g., De Fruyt et al., 2006), (2) are widely employed and thus can be compared to different literatures (e.g., Furr, 2008; Sherman, Nave, \& Funder, 2012; Wood \& Furr, 2016), and (3) perform favorably compared to other indices (McCrae, 2008; Rounds et al., 1987).

A profile-approach to person-environment fit is person-oriented because each person idiographically obtains a $q$-correlation score. When several persons are sampled, this also makes it possible to nomothetically quantify inter-individual differences in person-environment fit and position them in nomological networks (i.e., examining their causes/antecedents, concomitants, and consequences). As can be seen in the PERM (Figure 2; Table 4), the person-environment correlation or fit may be predicted from prior navigation mechanisms (paths t', $\mathrm{t}^{\prime \prime}$ ), outcome variables (path s), and itself (path m) over time, while also predicting later variables (path e). If profile fits are computed for a number of measurement occasions for each individual, then intra-individual density distributions in person-environment fit can be obtained. An example of such a withinperson distribution of $q$-correlations is given in the left panel in Figure 5. For each person, different density distribution parameters (e.g., mean, median, standard deviation, minimum, maximum, skewness, kurtosis) are available to be explored. This also means that in a sample of several persons, single persons or groups of persons can be compared in their density distribution parameters (see middle panel in Figure 5), revealing interindividual or group-level differences. Further, obtaining $q$-correlations in cross-sectional data for persons from different ages (e.g., 15-80 years) can yield a lifespan perspective on mean levels of person-environment fit (see right panel in Figure 5). Obtaining $q$ correlations from longitudinal data (i.e., at least two measurement points) for persons from different ages can yield a lifespan perspective on how inter-individual differences in person-environment fit among the sampled persons are conserved across time. Such data allow the examination of the levels of (1) rank-order stability in general, (2) rank-order stability in relation to age, and (3) inter-individual differences in intra-individual change of person-environment fit (which could then also potentially be explained or predicted by other variables). To my knowledge, no study has examined these questions yet.

Regularly computed "overall” $q$-correlations between "raw" profiles contain a certain amount of normativity (Cronbach, 1955; Cronbach \& Gleser, 1953; Furr, 2008) 
which can artificially inflate their size and also create misleadingly positive correlations with external variables (Wood \& Furr, 2016). For example, an overall profile correlation may be high because persons in the sample are like normative persons with a typical variable profile who encounter normative environments with a typical variable profile. Wood and Furr (2016) thus recommend a two-pronged data-analytical strategy of reporting overall profile correlations containing normativity and distinctive profile correlations controlling for normativity. An index of overall fit is simply the regular $q$ correlation, which will be high to some degree simply because the to-be-correlated profiles are each similar to normative profiles (e.g., where each element of the profile is a mean across many and ideally representative persons or environments). Normativity captures what is typical (or deemed socially acceptable or desirable) in a given socioculture. However, persons and environments may deviate from existing norms, and such deviations may be psychologically meaningful. A distinctive profile correlation is obtained when one first controls in both to-be-correlated profiles for their respective normative profiles and then correlates the resulting two residual profiles. Overall profile correlations confound both normative and distinctive aspects; by additionally quantifying distinctive profile correlations, the contribution of normativity can be made apparent. Thus, there are actually three kinds of profile-based person-environment fit: overall, normative, and distinctive fit. Importantly, these cannot be ordinarily disentangled with a variable-oriented approach but only within a profile-oriented one. ${ }^{23}$

\footnotetext{
${ }^{23}$ The procedure of obtaining overall and distinctive profile correlations is somewhat inelegant because it requires several piecemeal steps (especially when predictors, correlates, or outcomes are introduced) and does not provide any direct quantification of normative person-environment fit. A more powerful modeling procedure based on Biesanz' (2010) Social Accuracy Model and relying on random-coefficients modeling of profiles (level 1) nested within individuals (level 2) can be devised, though it has to date not been used in any empirical research yet (for details, see Rauthmann, Horstmann, and Sherman, in prep.), rests on several assumptions that may not be easily met, and is more difficult to understand.
} 


\section{Box 4 \\ Analysis of Cross-lagged Person-Environment Transactions}

The PERM in Figure 2 contains a cross-lagged model where a predictor at T1 predicts a criterion at $\mathrm{T} 2$ while controlling for the criterion at $\mathrm{T} 1$. Reversing the roles of predictors and criterion can help establish a lead-lag relationship (Finkel, 1995). These sorts of analyses can be conducted with multiple linear regressions or structural equation modeling (path analysis). However, there are several improvements to the basic idea of cross-lagged analyses available. For example, there are cross-lagged longitudinal mediation models (Cole \& Maxwell, 2003) and advancements of and alternatives to cross-lagged models (Mund \& Nestler, 2019). Further, high-intensity longitudinal measurements across time (Horstmann, Chapter 30) allow for more dynamic or even continuous modeling of the data (e.g., Costantini, Chapter 39; Geiser et al., Chapter 37; Driver \& Völkle, Chapter 34; Michaels et al., Chapter 38; Sadikaj et al., Chapter 33; Völkle et al., 2019; Wagner, Lüdtke, \& Völkle, 2019). Additionally, it is important to distinguish between within- and between-person effects (Asendorpf, Chapter 31; Hamaker, Kuiper, \& Grasman, 2015). Further, different instantiations of longitudinal models to study reciprocal relations exist, and these can all be couched within a single unified framework (see Usami, Murayama, \& Hamaker, 2019). 


\section{Box 5}

\section{Example of Corresponsive Personality Change via Person-Environment Relations}

The PERM in Figure 2 is a macroscopic model that can be further concretized for different research purposes. For example, it can be reassembled to capture downstream effects of person-environment fit (Figure 6). However, the PERM can also be rearranged by dropping the outcome variables and distinguishing different hierarchically nested person and environment variables (short-term, momentary: P, E; middle-term, aggregated: $\overline{\mathrm{P}}, \overline{\mathrm{E}}$; long-term, enduring: p, e; constraints, often individually immutable: genome g, culture c) and additionally combining Figures 7 and 8 into it. Such a reworking yields Figure 10 which is better equipped to track processes, sequences, and cycles more easily. Figure 10 is already filled with an illustrative example for a corresponsive pattern of personality change (see discussion in 4.4.1.1 Ontogenetic Processes).

There are some particularities of the model in Figure 10 that need to be noted before describing an exemplary corresponsive pattern. First, the hierarchical system is multi-determined, multi-final, and multi-functional. The depiction is, however, already simplified. Second, some effects (e.g., from g to P) have to "trickle down" via multiple intermediate levels (e.g., gene expression, cell structures, neuroanatomy, biochemical processes, etc.). Indeed, there are only few direct effects in the model. Most effects will be indirect or mediated by several variables, thus creating temporal and/or causal sequences or chains. Conversely, across time effects may accumulate and "trickle up" (e.g., repeated $\mathrm{P}$ sequences, or $\overline{\mathrm{P}}$, coalescing into $\mathrm{p}$ ). It will be rare though not entirely impossible that a short-term variable has a direct and immediate effect on a long-term one (with exceptions such as brain damage after a car crash, intense bouts of violence inducing psychological trauma, etc.). However, these direct, timescale-asymmetrical effects are not depicted in Figure 10 because they are either not the norm or hard to track. Repeated enactments of ABCDs (affects, behaviors, cognitions, desires; Wilt \& Revelle, 2015 ) or exposure to situations will, in most cases, have effects on more enduring 
variables or even constitute these. ${ }^{24}$ Third, the model contains effects occurring within or across different timescales, capturing ontogenetic, manifestive, actualgenetic, and accretive processes. These processes may interlock to bring forth different patterns of interest (e.g., a corresponsive pattern of personality change). Thus, patterns can be traced within this model and associated processes specified more easily.

The red highlighted paths in Figure 10 represent an exemplary tracing of a corresponsive personality change effect unfolding across time. Consider the red highlighted paths from the left (Points 0' and 0") to the right (Point 11). First, a trait p is to some degree a function of, and bound by, a genome and genetic activity g (Point $0^{\prime}$ ). The trait $\mathrm{p}$ is related to habitual ABCDs $\overline{\mathrm{P}}$ in daily life (Point 1 ) which are actualgenetically manifested in several specific ABCD states $\mathrm{P}$ at each moment (Point 2). These states $\mathrm{P}$ show some consistency and stability, and they are also actualgenetically related to momentary situation characteristics E (Point 3). Specifically, there are bidirectional transactions between $\mathrm{P}$ and $\mathrm{E}$ variables as well as (later) also correlations among them (Point 3). The E variables, in turn, are related to habitual environment characteristics $\bar{E}$ (Point 4) which are themselves related to even more enduring environment characteristics e (Point 5). The latter, in turn, are embedded into a cultural and historical context c (Point 0") - analogous to how experiences and behavior are embedded into the genetic, neurological, and biophysiological functioning of persons (Point $0^{\prime}$ ). The enduring environment characteristics e show some stability across time (Point 6), and the later ones are related to later instances of $\bar{E}$ (Point 7). Those $\bar{E}$ variables, in turn, are related to actualgenetic E instantiations (Point 8) which show some consistency and stability and also begin to exert direct effects on later P states (Point 9). Those $\mathrm{P}$ states, then, also show some consistency and stability and are related again to a habitual $\overline{\mathrm{P}}$ (Point 10). That habitual $\overline{\mathrm{P}}$ is part of the now changed trait $\mathrm{p}$ (Point 11). Tracing the red highlights thus granted the opportunity to pinpoint how a trait changed sequentially in a corresponsive way via 11 consecutive steps. Of course, this served just

\footnotetext{
${ }^{24}$ For example, each $\mathrm{P}$ could be seen as caused by an underlying $\mathrm{p}$ (reflective model), forming the $\mathrm{p}$ (formative model), or being functionally related within a complex network where temporally stable structures and dynamics constitute $\mathrm{p}$ as an emergent phenomenon (network model). Thus, there are different perspectives that can be invoked (Fried, 2017), and each of them come with different measurement and data modeling assumptions. Figure 10 thus only contains arrows if the causal direction is clear(er) and/or temporally warranted (i.e., prior variables predict later ones).
} 
as an illustrative example, and there are also several other ways how corresponsive patterns could emerge.

Taken together, as can be seen in Figure 10, something as simple as a corresponsive effect (i.e., traits craft niches which then change traits) is a function of several concatenated effects. One can zoom into the variables, effects, and sequences contained in Figure 10 even more. For example, each $\mathrm{P}$ variable actually represents different $\mathrm{ABCD}$ s that stand in certain relations to each other (e.g., in a network: Cramer et al., 2012). Further, each path or effect (e.g., the arrows) can reflect different mechanisms going on (e.g., calibration types with associated navigation mechanisms). Lastly, each process may itself be constituted of a myriad of microscopic sub-processes (e.g., Baumert et al., 2017; Wrzus \& Roberts, 2017). Though there can never be a gapless tracing of everything involved, these further complexities should still be taken into account. Only then can we move towards more causal understandings of personenvironment relations (see 6.4 Explanation vs. Prediction and Causality). 
Table 1

Overview and Summary of PERM Concepts

\begin{tabular}{|c|c|c|c|c|}
\hline Concepts & Amount & Items & Table & Figure \\
\hline Basic elements & 4 & $\begin{array}{l}\text { - Person variables: } \mathbb{P}, \mathbb{P} \\
\text { - Environment variables: } \mathbb{E}, \mathbb{e}\end{array}$ & 2 & 2 \\
\hline Measurement flections & 2 & $\begin{array}{l}\text { - Once (single-moment) vs. repeated } \\
\text { - Variable- vs. profile-oriented }\end{array}$ & 2 & - \\
\hline Predictive combinations & 60 & Variations from $\mathbb{O}=f(\mathbb{P}, \mathbb{p}, \mathbb{E}, \mathbb{e})$ & 3 & - \\
\hline Effect paths & 20 & Paths a-t & 4 & 2 \\
\hline Person-environment relations & 4 & $\begin{array}{l}\text { - Interactions } \\
\text { - Correlations } \\
\text { - Fit } \\
\text { - Transactions }\end{array}$ & 4 & 2 \\
\hline $\begin{array}{l}\text { Approaches to person- } \\
\text { environment interactions }\end{array}$ & $3(+2)$ & $\begin{array}{l}\text { - Variance decomposition } \\
\text { - Linear } \\
\text { - Non-linear } \\
\text { - (Effect patterns) } \\
\text { - (Outcome-based person-environment fit) }\end{array}$ & 5 & 3,4 \\
\hline $\begin{array}{l}\text { Approaches to person- } \\
\text { environment correlations }\end{array}$ & 2 & $\begin{array}{l}\text { - Variable-oriented } \\
\text { - Person-/Profile-oriented }\end{array}$ & 6 & 5,6 \\
\hline $\begin{array}{l}\text { Approaches to person- } \\
\text { environment fit }\end{array}$ & 2 & $\begin{array}{l}\text { - Outcome-based, variable-oriented (simple } \\
\text { correlations; polynomial regression and } \\
\text { response surface analysis) } \\
\text { - Person-/Profile-oriented (profile } \\
\text { correlations: overall, normative, } \\
\text { distinctive) }\end{array}$ & - & $4 d, 5,6$ \\
\hline $\begin{array}{l}\text { Timescales of transaction } \\
\text { processes }\end{array}$ & 4 & $\begin{array}{l}\text { - Ontogenetic (long } \rightarrow \text { long) } \\
\text { - Manifestive/expressive (long } \rightarrow \text { short) } \\
\text { - Actualgenetic (short } \rightarrow \text { short) } \\
\text { - Accretive/accumulative (short } \rightarrow \text { long) }\end{array}$ & - & 7 \\
\hline $\begin{array}{l}\text { Effect patterns of person- } \\
\text { environment transactions }\end{array}$ & 16 & $\begin{array}{l}\text { - Non-transaction patterns }(4 \mathrm{x}) \\
\text { - Bidirectional transaction patterns }(4 \mathrm{x}) \\
\text { - Monodirectional transaction patterns }(8 \mathrm{x})\end{array}$ & 7 & 8 \\
\hline Calibration types & 4 & $\begin{array}{l}\text { - Person } \rightarrow \text { Person } \\
\text { - Person } \rightarrow \text { Environment } \\
\text { - Environment } \rightarrow \text { Person } \\
\text { - Environment } \rightarrow \text { Environment }\end{array}$ & 8 & - \\
\hline $\begin{array}{l}\text { Person } \rightarrow \text { Environment } \\
\text { navigation mechanisms }\end{array}$ & 6 & $\begin{array}{l}\text { - Maintenance } \\
\text { - Construal } \\
\text { - Evocation } \\
\text { - Selection } \\
\text { - Modulation } \\
\text { - Creation } \\
\end{array}$ & 8,9 & 9 \\
\hline $\begin{array}{l}\text { Recommendation and } \\
\text { suggestions }\end{array}$ & 10 & $\begin{array}{l}\text { 1. Taking the assessment of environment } \\
\text { variables seriously } \\
\text { 2. Employing appropriate research designs }\end{array}$ & - & - \\
\hline
\end{tabular}


3. Zooming into processes, sequences, and cycles

4. Attending to explanation vs. prediction and causality

5. Conceptualizing, measuring, and studying calibration and navigation mechanisms

6. Distinguishing different types of personenvironment fit clearly

7. Devising a transactional perspective on traits

8. Providing (mathematical) formalizations

9. Increasing efforts for theory, integration, replication, and collaboration

10. Working towards principles of personenvironment relations 


\section{Table 2}

Basic Elements and their Flections

\begin{tabular}{|c|c|c|c|c|c|}
\hline \multirow[b]{2}{*}{ Element } & \multirow[b]{2}{*}{ Description } & \multicolumn{4}{|c|}{ Attributes } \\
\hline & & $\begin{array}{l}\text { Variable } \\
\text { Group }\end{array}$ & $\begin{array}{l}\text { Time- } \\
\text { scale }\end{array}$ & $\begin{array}{l}\text { Measurement } \\
\text { Occasions }\end{array}$ & $\begin{array}{l}\text { Measurement } \\
\text { Orientation }\end{array}$ \\
\hline $\mathbb{P}$ & Placeholder for any kind of short-term person variables (one or several) & Person & Short-term & Any & Any \\
\hline p & Placeholder for any kind of long-term person variables (one or several) & Person & Long-term & Any & Any \\
\hline $\mathbb{E}$ & Placeholder for any kind of short-term environment variables (one or several) & Environment & Short-term & Any & Any \\
\hline e & Placeholder for any kind of long-term environment variables (one or several) & Environment & Long-term & Any & Any \\
\hline $\mathrm{P}$ & Single short-term person variable measured once in time & Person & Short-term & Single-time & Variable (Single) \\
\hline $\mathrm{p}$ & Single long-term person variable measured once in time & Person & Long-term & Single-time & Variable (Single) \\
\hline $\mathrm{E}$ & Single short-term environment variable measured once in time & Environment & Short-term & Single-time & Variable (Single) \\
\hline $\mathrm{e}$ & Single long-term environment variable measured once in time & Environment & Long-term & Single-time & Variable (Single) \\
\hline$\overline{\mathrm{P}}$ & $\begin{array}{l}\text { Density distribution parameter or latent score of a repeatedly measured single } \\
\text { short-term person variable }\end{array}$ & Person & Short-term & Repeatedly & Variable (Single) \\
\hline$\overline{\mathrm{p}}$ & $\begin{array}{l}\text { Density distribution parameter or latent score of a repeatedly measured single } \\
\text { long-term person variable }\end{array}$ & Person & Long-term & Repeatedly & Variable (Single) \\
\hline$\overline{\mathrm{E}}$ & $\begin{array}{l}\text { Density distribution parameter or latent score of a repeatedly measured single } \\
\text { short-term environment variable }\end{array}$ & Environment & Short-term & Repeatedly & Variable (Single) \\
\hline$\overline{\mathrm{e}}$ & $\begin{array}{l}\text { Density distribution parameter or latent score of a repeatedly measured single } \\
\text { long-term environment variable }\end{array}$ & Environment & Long-term & Repeatedly & Variable (Single) \\
\hline$\dddot{\mathrm{P}}$ & $\begin{array}{l}\text { Profile of several different short-term person variables, each measured once in } \\
\text { time }\end{array}$ & Person & Short-term & Single-time & Person (Profile) \\
\hline$\dddot{\mathrm{p}}$ & $\begin{array}{l}\text { Profile of several different long-term person variables, each measured once in } \\
\text { time }\end{array}$ & Person & Long-term & Single-time & Person (Profile) \\
\hline$\dddot{\mathrm{E}}$ & $\begin{array}{l}\text { Profile of several different short-term environment variables, each measured once } \\
\text { in time }\end{array}$ & Environment & Short-term & Single-time & Person (Profile) \\
\hline$\dddot{\mathrm{e}}$ & $\begin{array}{l}\text { Profile of several different long-term environment variables, each measured once } \\
\text { in time }\end{array}$ & Environment & Long-term & Single-time & Person (Profile) \\
\hline$\dddot{\overline{\mathrm{P}}}$ & $\begin{array}{l}\text { Profile of density distribution parameters or latent scores of several different } \\
\text { short-term person variables, each measured repeatedly }\end{array}$ & Person & Short-term & Repeatedly & Person (Profile) \\
\hline$\dddot{\overline{\mathrm{p}}}$ & $\begin{array}{l}\text { Profile of density distribution parameters or latent scores of several different long- } \\
\text { term person variables, each measured repeatedly }\end{array}$ & Person & Long-term & Repeatedly & Person (Profile) \\
\hline$\dddot{\bar{E}}$ & $\begin{array}{l}\text { Profile of density distribution parameters or latent scores of several different } \\
\text { short-term environment variables, each measured repeatedly }\end{array}$ & Environment & Short-term & Repeatedly & Person (Profile) \\
\hline$\dddot{\mathrm{e}}$ & $\begin{array}{l}\text { Profile of density distribution parameters or latent scores of several different long- } \\
\text { term environment variables, each measured repeatedly }\end{array}$ & Environment & Long-term & Repeatedly & Person (Profile) \\
\hline
\end{tabular}

Note. Outcome variables $\mathbb{O}$ may be defined as any $\mathbb{P}, \mathbb{P}, \mathbb{E}$, or $\mathbb{e}$ variables. 
Table 3

Basic Predictive Combinations

\begin{tabular}{|c|c|c|c|c|}
\hline Formula & $\begin{array}{c}\text { Predictor } \\
\text { Variables }^{\mathrm{a}}\end{array}$ & $\begin{array}{c}\text { Prediction } \\
\text { Type }^{\mathrm{b}}\end{array}$ & $\begin{array}{l}\text { Element } \\
\text { Balance }^{c}\end{array}$ & $\begin{array}{c}\text { Time } \\
\text { Symmetry }^{\mathrm{d}}\end{array}$ \\
\hline $\mathbb{O}=f(\mathbb{P})$ & Person-only & One-way & - & - \\
\hline $\mathbb{O}=f(\mathbb{P})$ & Person-only & One-way & - & - \\
\hline $\mathbb{Q}=f(\mathbb{p}, \mathbb{P})$ & Person-only & Two-way & - & No \\
\hline $\mathbb{0}=f(\mathbb{e})$ & Environment-only & One-way & - & - \\
\hline $\mathbb{0}=f(\mathbb{E})$ & Environment-only & One-way & - & - \\
\hline $\mathbb{O}=f(e, \mathbb{E})$ & Environment-only & Two-way & - & No \\
\hline $\mathbb{O}=f(\mathbb{P}, \mathbb{e})$ & Mixed & Two-way & Yes & Yes \\
\hline $\mathbb{0}=f(\mathbb{p}, \mathbb{E})$ & Mixed & Two-way & Yes & No \\
\hline $\mathbb{0}=f(\mathbb{P}, \mathbb{e})$ & Mixed & Two-way & Yes & No \\
\hline $\mathbb{O}=f(\mathbb{P}, \mathbb{E})$ & Mixed & Two-way & Yes & Yes \\
\hline $\mathbb{O}=f(\mathbb{p}, \mathbb{e}, \mathbb{E})$ & Mixed & Three-way & No & No \\
\hline $\mathbb{O}=f(\mathbb{P}, \mathbb{e}, \mathbb{E})$ & Mixed & Three-way & No & No \\
\hline $\mathbb{O}=f(\mathbb{P}, \mathbb{P}, \mathbb{e})$ & Mixed & Three-way & No & No \\
\hline $\mathbb{O}=f(\mathbb{p}, \mathbb{P}, \mathbb{E})$ & Mixed & Three-way & No & No \\
\hline $\mathbb{O}=f(\mathbb{P}, \mathbb{P}, \mathbb{E}, \mathbb{e})$ & Mixed & Four-way & Yes & Yes \\
\hline
\end{tabular}

Note. The letters with double lines $(\mathbb{O}, \mathbb{P}, \mathbb{p}, \mathbb{E}, \mathbb{e})$ denote sets of variables that are undifferentiated placeholders that could be any form of a specific variable. For example, $\mathbb{P}$ refers to any kind of momentary person variable (and could denote $\mathrm{P}, \overline{\mathrm{P}}, \dddot{\mathrm{P}}$, or $\dddot{\overline{\mathrm{P}}}$; see Table 2 ), while a P refers to a specific momentary person variable measured once in time at one given measurement occasion.

Outcome variables $\mathbb{O}$ may be defined as any $\mathbb{P}, \mathbb{P}, \mathbb{E}$, or $\mathbb{e}$ variables. Taking this into account, there are actually $4 \times 15=60$ different predictive combinations possible. Note further that any of the predictors $(\mathbb{P}, \mathbb{P}, \mathbb{E}$, or $\mathbb{e})$ represent sets and thus could stand for only one predictor or multiple predictors of the same element (e.g., $\mathbb{P}_{1}, \mathbb{P}_{1}, \ldots, \mathbb{P}_{n}$ ) in each equation.

To obtain a palpable example of how the formula could look in each instance, following sample variables may be plugged in: $\mathbb{O}=$ making new friends, $\mathbb{P}=$ pleasant mood, $\mathbb{P}=$ trait extraversion, $\mathbb{E}=$ party, $\mathbb{e}=$ social network.

${ }^{\text {a }}$ Predictor variables: Are person and/or environment variables, respectively, used as predictors?

${ }^{\mathrm{b}}$ Prediction type: How many distinct sets of person and/or environment variables, respectively, are used as predictors?

${ }^{c}$ Element balance: Are person and environment variables (the elements) represented to equal amounts?

d Time symmetry: Are the person and environment variables (or sets of variables), respectively, either defined both as short-term or both as long-term variables, respectively? In other words: Do the timescales of the predictor variables match up? 


\section{Table 4}

Summary of PERM Effect Paths

\begin{tabular}{|c|c|c|c|c|c|}
\hline $\begin{array}{l}\text { Effect } \\
\text { Path }\end{array}$ & Variables & Description $^{\mathrm{a}}$ & Type & Timeframe & $\begin{array}{l}\text { Person-Environment } \\
\text { Relation }\end{array}$ \\
\hline $\mathrm{a}$ & Person $_{n} \rightarrow$ Outcome $_{n}$ & $\begin{array}{l}\text { A person variable is concurrently } \\
\text { associated with (predicts) an } \\
\text { outcome variable. }\end{array}$ & Prediction & Concurrent & \\
\hline$b$ & Environment $_{n} \rightarrow$ Outcome $_{n}$ & $\begin{array}{l}\text { An environment variable is } \\
\text { concurrently associated with } \\
\text { (predicts) an outcome variable. }\end{array}$ & Prediction & Concurrent & Interaction \\
\hline$c^{\prime}$ & Environment $_{n} \rightarrow\left(\right.$ Person $_{n} \rightarrow$ Outcome $\left._{n}\right)$ & $\begin{array}{l}\text { An environment variable moderates } \\
\text { the effect of a person variable on an } \\
\text { outcome variable. }\end{array}$ & Moderation & Concurrent & (Fit) \\
\hline c" & Person $_{n} \rightarrow\left(\right.$ Environment $_{n} \rightarrow$ Outcome $\left._{n}\right)$ & $\begin{array}{l}\text { A person variable moderates the } \\
\text { effect of an environment variable } \\
\text { on an outcome variable. }\end{array}$ & Moderation & Concurrent & \\
\hline d & Person $_{n} \leftrightarrow$ Environment $_{n}$ & $\begin{array}{l}\text { A person variable is correlated with } \\
\text { an environment variable. }\end{array}$ & Correlation & Concurrent & $\begin{array}{l}\text { Correlation } \\
\text { (Fit) }\end{array}$ \\
\hline $\mathrm{e}$ & $\left(\right.$ Person $_{n} \leftrightarrow$ Environment $\left._{n}\right) \rightarrow$ Outcome $_{n}$ & $\begin{array}{l}\text { A person-environment correlation } \\
\text { is associated with (predicts) an } \\
\text { outcome variable. }\end{array}$ & Prediction & Concurrent & - \\
\hline $\mathrm{f}^{\prime}, \mathrm{f}^{\prime \prime}$ & Mechanism $_{n} \rightarrow\left(\right.$ Person $_{n} \leftrightarrow$ Environment $\left._{n}\right)$ & $\begin{array}{l}\text { A mechanism variable is } \\
\text { concurrently associated with } \\
\text { (predicts) a person-environment } \\
\text { correlation. }\end{array}$ & Prediction & Concurrent & - \\
\hline$g^{\prime}$ & Mechanism $_{n}-$ Person $_{n}$ & $\begin{array}{l}\text { A mechanism variable originates } \\
\text { from or is tied to a person. }\end{array}$ & Origination $^{\mathrm{b}}$ & Concurrent & - \\
\hline g" & Mechanism $_{n}-$ Environment $_{n}$ & $\begin{array}{l}\text { A mechanism variable originates } \\
\text { from or is tied to an environment. }\end{array}$ & Origination $^{b}$ & Concurrent & - \\
\hline $\mathrm{h}$ & Mechanism $_{n} \rightarrow$ Outcome $_{n}$ & $\begin{array}{l}\text { A mechanism variable is } \\
\text { concurrently associated with } \\
\text { (predicts) an outcome variable. }\end{array}$ & Prediction & Time-lagged & - \\
\hline$i^{\prime}, i^{\prime \prime}$ & Mechanism $_{n} \rightarrow$ Mechanism $_{n+1}$ & $\begin{array}{l}\text { A prior mechanism variable } \\
\text { predicts itself at a later time-point. }\end{array}$ & Stability/Change & Time-lagged & - \\
\hline $\mathrm{j}$ & Person $_{n} \rightarrow$ Person $_{n+1}$ & $\begin{array}{l}\text { A prior person variable predicts } \\
\text { itself at a later time-point. }\end{array}$ & Stability/Change & Time-lagged & - \\
\hline $\mathrm{k}$ & Environment $_{n} \rightarrow$ Environment $_{n+1}$ & $\begin{array}{l}\text { A prior environment variable } \\
\text { predicts itself at a later time-point. }\end{array}$ & Stability/Change & Time-lagged & - \\
\hline
\end{tabular}




\begin{tabular}{|c|c|c|c|c|c|}
\hline $\mathrm{m}$ & $\begin{array}{l}\text { Outcome }_{n} \rightarrow \text { Outcome }_{n+1} \\
\left(\text { Person }_{n} \leftrightarrow \text { Environment }_{n}\right) \rightarrow \\
\left(\text { Person }_{n+1} \leftrightarrow \text { Environment }_{n+1}\right)\end{array}$ & $\begin{array}{l}\text { A prior outcome variable predicts } \\
\text { itself at a later time-point. } \\
\text { A prior person-environment } \\
\text { correlation predicts itself at a later } \\
\text { time-point. }\end{array}$ & Stability/Change & Time-lagged & - \\
\hline $\mathrm{n}$ & $\begin{array}{l}\text { Person }_{n} \rightarrow \text { Environment }_{n+1} \\
\text { Environment }_{n} \rightarrow \text { Person }_{n+1}\end{array}$ & $\begin{array}{l}\text { A prior person variable predicts a } \\
\text { later environment variable. } \\
\text { A prior environment variable } \\
\text { predicts a later person variable. }\end{array}$ & $\begin{array}{l}\text { Prediction } \\
\text { Prediction }\end{array}$ & $\begin{array}{l}\text { Time-lagged } \\
\text { Time-lagged }\end{array}$ & Transaction \\
\hline $\mathrm{p}^{\prime}, \mathrm{p}^{\prime \prime}$ & Outcome $_{n} \rightarrow$ Mechanism $_{n+1}$ & $\begin{array}{l}\text { A prior outcome variable predicts a } \\
\text { later mechanism variable. } \\
\text { A prior outcome variable predicts a }\end{array}$ & Prediction & Time-lagged & - \\
\hline q & Outcome $_{n} \rightarrow$ Person $_{n+1}$ & $\begin{array}{l}\text { A prior outcome variable predicts a } \\
\text { later person variable. }\end{array}$ & Prediction & Time-lagged & - \\
\hline $\mathrm{r}$ & Outcome $_{n} \rightarrow$ Environment $_{n+1}$ & $\begin{array}{l}\text { A prior outcome variable predicts a } \\
\text { later environment variable. }\end{array}$ & Prediction & Time-lagged & - \\
\hline $\mathrm{s}$ & Outcome $_{n} \rightarrow\left(\right.$ Person $_{n+1} \leftrightarrow$ Environment $\left._{n+1}\right)$ & $\begin{array}{l}\text { A prior outcome variable predicts a } \\
\text { later person-environment } \\
\text { correlation. }\end{array}$ & Prediction & Time-lagged & - \\
\hline $\mathrm{t}^{\prime}, \mathrm{t}^{\prime \prime}$ & Mechanism $_{n} \rightarrow\left(\right.$ Person $_{n+1} \leftrightarrow$ Environment $\left._{n+1}\right)$ & $\begin{array}{l}\text { A prior mechanism variable } \\
\text { predicts a later person-environment } \\
\text { correlation }\end{array}$ & Prediction & Time-lagged & - \\
\hline
\end{tabular}

Note. PERM = Person-Environment Relations Model. For an integrated graphical depiction of the PERM effects, see Figure 2.

${ }^{a}$ The descriptions are all framed in a variable-oriented manner, but for some effects (especially person-environment correlations and fits) person- or profile-oriented views are also possible and sensible.

${ }^{\mathrm{b}}$ Navigation mechanisms can originate from persons (signified by ' in relevant paths) if they are geared towards a person $\rightarrow$ environment calibration. In the same manner, navigation mechanisms can also originate from environments (signified by " in relevant paths) if they are geared towards an environment $\rightarrow$ person calibration. All paths involving navigation mechanisms can thus exist at the person (left in Figure 2, with ') and at the environment side (right in Figure 2, with "). 
Table 5

Overview of Selected Person-Environment-Outcome Effect Patterns

\begin{tabular}{|c|c|c|c|c|c|c|}
\hline \multicolumn{2}{|l|}{ Patterns } & \multicolumn{5}{|c|}{ Presence of PERS and ENV Predictor Effects } \\
\hline Label & Krueger (2009) & PERS & ENV & $\mathrm{PERS} \times \mathrm{ENV}$ & $\mathrm{PERS}^{2}$ & $\mathrm{ENV}^{2}$ \\
\hline \multicolumn{7}{|l|}{ Linear Patterns } \\
\hline Zero (Intercept-only) & Wash-out & & & & & \\
\hline Person-only & Disposition only & $\bullet$ & & & & \\
\hline Environment-only & Situation only & & $\bullet$ & & & \\
\hline Additive & Non-hydraulic & $\bullet$ & $\bullet$ & & & \\
\hline \multicolumn{7}{|l|}{ Interactionist Patterns } \\
\hline Interaction-only & Interaction only & & & $\bullet$ & & \\
\hline Person plus Interaction & Classic trait & $\bullet$ & & $\bullet$ & & \\
\hline Environment plus Interaction & Classic social & & $\bullet$ & $\bullet$ & & \\
\hline Additive plus Interaction & Complexity & $\bullet$ & $\bullet$ & $\bullet$ & & \\
\hline \multicolumn{7}{|l|}{ Non-linear Patterns } \\
\hline Person Strength & - & & & & $\bullet$ & \\
\hline Environment Strength & - & & & & & $\bullet$ \\
\hline Non-linear Additive & - & & & & $\bullet$ & $\bullet$ \\
\hline 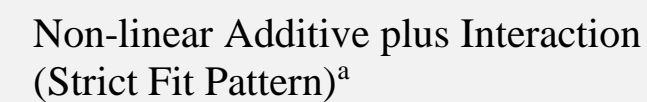 & - & & & $\bullet$ & • & $\bullet$ \\
\hline Full Complexity Pattern & - & $\bullet$ & $\bullet$ & $\bullet$ & $\bullet$ & $\bullet$ \\
\hline
\end{tabular}

Note. PERS = any person variable (either $\mathbb{P}$ or $\mathbb{P}), E N V=$ any environment variable (either $\mathbb{E}$ or $\mathbb{e}$ ).

- = statistically significant, sizable, or otherwise meaningful effect of a predictor.

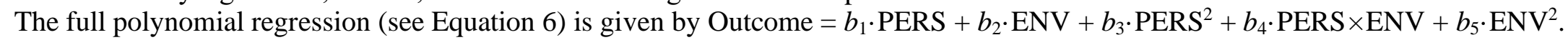

Response surface parameters: $a_{1}=b_{1}+b_{2} ; a_{2}=b_{3}+b_{4}+b_{5} ; a_{3}=b_{1}-b_{2} ; a_{4}=b_{3}-b_{4}+b_{5}$.

Samples of all patterns are depicted in Figures $4 \mathrm{a}-\mathrm{d}$.

a See the first panel of Figure 4d under "Strict Fit Pattern". 
Table 6

Possible Types of Person-Environment Correlations

\begin{tabular}{|c|c|c|c|c|}
\hline \multicolumn{2}{|c|}{ Correlation Between } & \multirow{2}{*}{ Symmetry $^{a}$} & \multirow{2}{*}{ Type $^{\text {b }}$} & \multirow{2}{*}{ Correlation } \\
\hline Person & Environment & & & \\
\hline \multicolumn{5}{|c|}{ Variable-oriented } \\
\hline $\mathrm{P}$ & $\mathrm{E}$ & Yes & State & $R$ \\
\hline $\mathrm{P}$ & $\overline{\mathrm{E}}$ & No & Mixed & $R$ \\
\hline $\mathrm{P}$ & $\mathrm{e}$ & No & Mixed & $R$ \\
\hline $\mathrm{P}$ & $\overline{\mathrm{e}}$ & No & Mixed & $R$ \\
\hline$\overline{\mathrm{P}}$ & $\mathrm{E}$ & No & Mixed & $R$ \\
\hline$\overline{\mathrm{P}}$ & $\overline{\mathrm{E}}$ & Yes & State-Aggregate & $R$ \\
\hline$\overline{\mathrm{P}}$ & e & No & Mixed & $R$ \\
\hline$\overline{\mathrm{P}}$ & $\overline{\mathrm{e}}$ & Semi & Mixed & $R$ \\
\hline $\mathrm{p}$ & $\mathrm{E}$ & No & Mixed & $R$ \\
\hline $\mathrm{p}$ & $\overline{\mathrm{E}}$ & Semi & Mixed & $R$ \\
\hline $\mathrm{p}$ & e & Yes & Trait & $R$ \\
\hline $\mathrm{p}$ & $\overline{\mathrm{e}}$ & Semi & Mixed & $R$ \\
\hline$\overline{\mathrm{p}}$ & $\mathrm{E}$ & No & Mixed & $R$ \\
\hline$\overline{\mathrm{p}}$ & $\overline{\mathrm{E}}$ & Semi & Mixed & $R$ \\
\hline$\overline{\mathrm{p}}$ & e & No & Mixed & $R$ \\
\hline$\overline{\mathrm{p}}$ & $\overline{\mathrm{e}}$ & Yes & Trait-Aggregate & $R$ \\
\hline \multicolumn{5}{|c|}{ Profile-oriented } \\
\hline$\dddot{\mathrm{P}}$ & $\dddot{\mathrm{E}}$ & Yes & State & $Q$ \\
\hline$\dddot{\mathrm{P}}$ & $\dddot{\overline{\mathrm{E}}}$ & No & Mixed & $Q$ \\
\hline$\dddot{\mathrm{P}}$ & $\dddot{\mathrm{e}}$ & No & Mixed & $Q$ \\
\hline$\dddot{\mathrm{P}}$ & $\dddot{\mathrm{e}}$ & No & Mixed & $Q$ \\
\hline$\dddot{\overline{\mathrm{P}}}$ & $\dddot{\mathrm{E}}$ & No & Mixed & $Q$ \\
\hline$\dddot{\overline{\mathrm{P}}}$ & $\dddot{\overline{\mathrm{E}}}$ & Yes & State-Aggregate & $Q$ \\
\hline$\dddot{\overline{\mathrm{P}}}$ & $\dddot{\mathrm{e}}$ & No & Mixed & $Q$ \\
\hline$\dddot{\overline{\mathrm{P}}}$ & $\dddot{\mathrm{e}}$ & Semi & Mixed & $Q$ \\
\hline$\dddot{\mathrm{p}}$ & $\dddot{\mathrm{E}}$ & No & Mixed & $Q$ \\
\hline$\dddot{\mathrm{p}}$ & $\dddot{\overline{\mathrm{E}}}$ & Semi & Mixed & $Q$ \\
\hline$\dddot{\mathrm{p}}$ & $\dddot{\mathrm{e}}$ & Yes & Trait & $Q$ \\
\hline$\dddot{\mathrm{p}}$ & $\dddot{\mathrm{e}}$ & Semi & Mixed & $\widetilde{Q}$ \\
\hline$\dddot{\overline{\mathrm{p}}}$ & $\dddot{\mathrm{E}}$ & No & Mixed & $Q$ \\
\hline$\dddot{\overline{\mathrm{p}}}$ & $\frac{\dddot{\mathrm{E}}}{\mathrm{m}}$ & Semi & Mixed & $Q$ \\
\hline$\dddot{\overline{\mathrm{p}}}$ & $\dddot{\mathrm{e}}$ & No & Mixed & $Q$ \\
\hline$\dddot{\overline{\mathrm{p}}}$ & $\dddot{\mathrm{e}}$ & Yes & Trait-Aggregate & $Q$ \\
\hline
\end{tabular}

Note. This table only contains correlations between person and environment variables (disregarding person-person and environment-environment correlations which are, of course, also possible). When the person and environment variables are contentwise commensurate or correspond in some meaningful way functionally to each other, then the correlations may denote fit.

${ }^{\mathrm{a}}$ Yes $=$ both variables are exactly on the same timescale; No $=$ both variables are at different timescales; Semi $=$ both variables are at approximately the same timescale (but not exactly the same).

${ }^{\mathrm{b}}$ Different types of variables may fit among or between each other (states, state-aggregates, traits).

${ }^{\mathrm{b}} R$-correlations are computed between two variables across a sample of persons (or environments). $Q$ correlations are computed between two profiles of variables within one person (or environment). 
Table 7

Overview of Different Types of Person-Environment Transaction Patterns

\begin{tabular}{|c|c|c|c|c|c|}
\hline \multirow{2}{*}{ Transaction Pattern } & \multicolumn{2}{|c|}{ Stability Paths ${ }^{\mathrm{a}}$} & \multicolumn{2}{|c|}{ Cross-lagged Paths ${ }^{\mathrm{a}}$} & \multirow{2}{*}{$\begin{array}{l}\text { Depiction } \\
\text { in Figure }\end{array}$} \\
\hline & PERS $_{n} \rightarrow$ PERS $_{n+1}$ & $\mathrm{ENV}_{n} \rightarrow \mathrm{ENV}_{n+1}$ & $\mathrm{PERS}_{n} \rightarrow \mathrm{ENV}_{n+1}$ & $\mathrm{ENV}_{n} \rightarrow \mathrm{PERS}_{n+1}$ & \\
\hline \multicolumn{6}{|l|}{ Non-Transactions } \\
\hline Zero Transaction & & & & & 1 \\
\hline Person Stability & $\bullet$ & & & & 2 \\
\hline Environment Stability & & $\bullet$ & & & 3 \\
\hline Bi-Stability & $\bullet$ & $\bullet$ & & & 4 \\
\hline \multicolumn{6}{|l|}{ Bidirectional Transactions } \\
\hline Full Bidirectional Transaction & $\bullet$ & $\bullet$ & $\bullet$ & $\bullet$ & 5 \\
\hline Reset Bidirectional Transaction & & & $\bullet$ & $\bullet$ & 6 \\
\hline Person-tilted Bidirectional Transaction & $\bullet$ & & $\bullet$ & $\bullet$ & 7 \\
\hline Environment-tilted Bidirectional Transaction & & $\bullet$ & $\bullet$ & $\bullet$ & 8 \\
\hline \multicolumn{6}{|l|}{ Monodirectional Transactions } \\
\hline \multicolumn{6}{|l|}{ from Persons to Environments } \\
\hline Person-tilted Monodirectional Transaction & $\bullet$ & $\bullet$ & $\bullet$ & & 9 \\
\hline Full Person Transaction & $\bullet$ & & $\bullet$ & & 10 \\
\hline Partial Person Transaction & & $\bullet$ & $\bullet$ & & 11 \\
\hline Weak Person Transaction & & & $\bullet$ & & 12 \\
\hline \multicolumn{6}{|l|}{ Monodirectional Transactions } \\
\hline \multicolumn{6}{|l|}{ from Environments to Persons } \\
\hline Environment-tilted Monodirectional Transaction & $\bullet$ & $\bullet$ & & $\bullet$ & 13 \\
\hline Full Environment Transaction & & $\bullet$ & & $\bullet$ & 14 \\
\hline Partial Environment Transaction & $\bullet$ & & & $\bullet$ & 15 \\
\hline Weak Environment Transaction & & & & $\bullet$ & 16 \\
\hline
\end{tabular}

Note. Indirect effects, curvilinear effects, and effects of variables from time $n+2$ etc. are not considered here. This means that even further, and more complex, patterns can be expected besides the 16 basic ones compiled in this table. Further, it is assumed that the effects do not vary across time.

The 16 transaction patterns are visualized in Figure 8.

${ }^{a}$ The stability paths (person variables: path $\mathrm{j}$; environment variables: path $\mathrm{k}$ ) and cross-lagged paths (person to environment variables: path $\mathrm{n}$; environment to person variables: path o) can be found in Figure 2. 
Table 8

Overview of Calibration Types and Associated Navigation Mechanisms

\begin{tabular}{ll}
\hline Calibration Type & Selected Potential Navigation Mechanisms \\
\hline Person $\rightarrow$ Person & Selection, Optimization, Compensation (SOC) Strategies \\
& Intentional personality change \\
& Self-regulation \\
\hline Person $\rightarrow$ Environment & Maintenance \\
& Construal \\
& Evocation \\
& Selection \\
& Modulation \\
& Creation \\
\hline Environment $\rightarrow$ Person & Transitioning \\
& Strength \\
& Nudging \\
& Attraction (drawing via rewards) vs. Attrition (repelling via punishments) \\
& Socialization \\
& Trauma \\
\hline Environment $\rightarrow$ & Chance \\
Environment & Autopoiesis \\
\hline
\end{tabular}

Note. For details on navigation mechanisms for person $\rightarrow$ environment calibrations, see Table 9 and Figure 9. 
Table 9

Navigation Mechanisms for Person $\rightarrow$ Environment Calibrations

\begin{tabular}{|c|c|c|c|c|c|c|}
\hline \multirow{2}{*}{$\begin{array}{l}\text { Navigation } \\
\text { Mechanism }\end{array}$} & \multirow[b]{2}{*}{ Description } & \multicolumn{5}{|c|}{ Other Literatures } \\
\hline & & $\begin{array}{l}\text { Plomin et al. } \\
\qquad(1977)^{\mathrm{a}}\end{array}$ & $\begin{array}{c}\text { Scarr \& McCartney } \\
(1983)^{\mathrm{b}}\end{array}$ & $\begin{array}{l}\text { Buss } \\
(1987)^{\mathrm{c}}\end{array}$ & $\begin{array}{l}\text { Caspi \& Roberts } \\
(2001)^{\mathrm{d}}\end{array}$ & $\begin{array}{c}\text { Roberts (2006), } \\
\text { Roberts \& Nickel (in press) }^{\mathrm{d}}\end{array}$ \\
\hline Maintenance & $\begin{array}{l}\text { Remaining passively in or } \\
\text { preserving an environment, thereby } \\
\text { maintaining it }\end{array}$ & $\begin{array}{l}\text { (Passive) } \\
\text { (Active) }\end{array}$ & $\begin{array}{c}\text { (Passive) } \\
\text { (Active) }\end{array}$ & - & - & - \\
\hline Construal & $\begin{array}{l}\text { Uniquely re-construing a perceived } \\
\text { environment differently than before }\end{array}$ & - & (Perception) & - & Reactive & - \\
\hline Evocation & $\begin{array}{l}\text { Willingly or unwillingly eliciting } \\
\text { certain reactions from the } \\
\text { environment }\end{array}$ & Reactive & Evocative & Evocation & Evocative & Evocation \\
\hline Selection & $\begin{array}{l}\text { Willingly avoiding one } \\
\text { environment and approaching or } \\
\text { entering another one }\end{array}$ & Active & Active & Selection & Pro-active & $\begin{array}{l}\text { Selection } \\
\text { Attrition } \\
\text { Attraction }\end{array}$ \\
\hline Modulation & $\begin{array}{l}\text { Actively modulating a previous } \\
\text { environment in a certain way so } \\
\text { that it changes into a different one }\end{array}$ & (Active) & (Active) & Manipulation & - & $\begin{array}{l}\text { Manipulation } \\
\text { Transformation }\end{array}$ \\
\hline Creation & $\begin{array}{l}\text { Pro-actively creating an entirely } \\
\text { new situation (from scratch) }\end{array}$ & (Active) & (Active) & Manipulation & - & Manipulation \\
\hline
\end{tabular}

Note. Modified from Tables 2 and 3 from Rauthmann \& Sherman (2016). See Figure 9 for an ordering of the person $\rightarrow$ environment navigation mechanisms and their relations to each other in a coherent system.

Parentheses ( ) mean that the respective term could potentially represent the navigation mechanism. A “-” means that there is likely no direct analog.

${ }^{a}$ genotype-environment correlation

${ }^{\mathrm{b}}$ gene $\rightarrow$ environment effects

${ }^{c}$ person-environment correspondence processes

${ }^{d}$ person-environment transactions 


\section{Figure 1}

Case Example for Unfolding Personality Dynamics

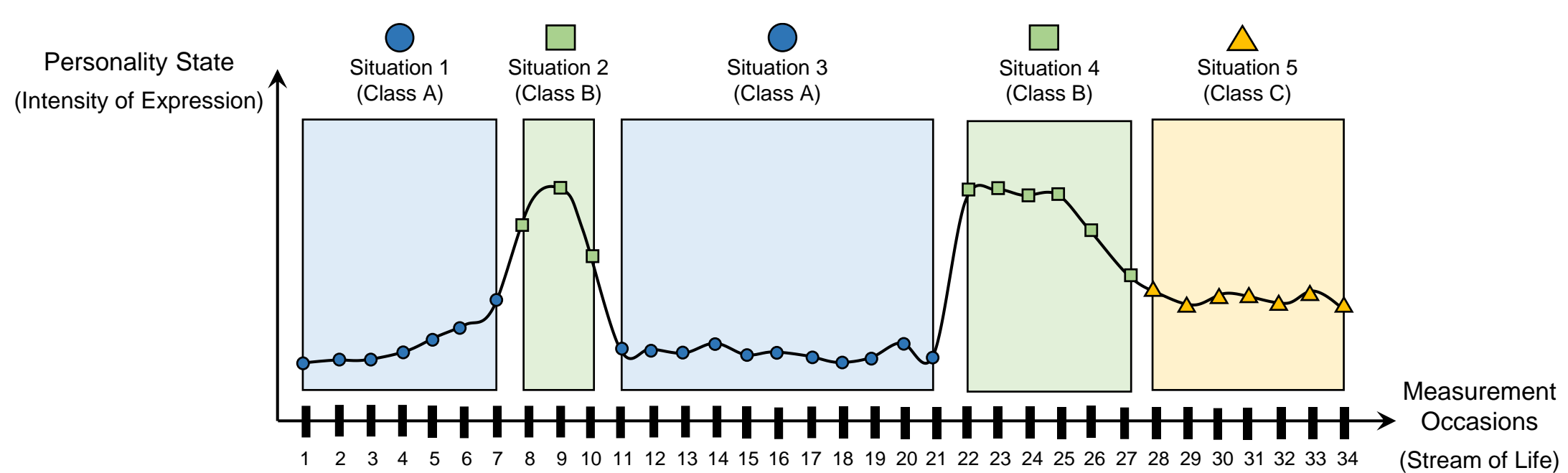

Note. Situations 1-5 are different situational episodes (shaded areas) adjacent to each other in a temporal sequence of 34 measurement occasions in the stream of life of one person, Anna. The five situational episodes further correspond to three classes of situations (A: blue dots; B: green boxes; C: yellow triangles). Situational episodes 1 and 2 belong to Class A, episodes 2 and 4 to Class B, and episode 5 to Class C. At each measurement occasion, a personality state's intensity of expression is measured (e.g., by experience sampling, behavioral observation). White or blank spaces between the shaded situational episodes denote transitions from one situational episode to another. The measured personality states show markedly different intensities at different measurement occasions. This fictitious example shows variations within situational episodes (e.g., expression intensity changes in situational episode 1, becoming more and more) and across situations (e.g., expression intensity is higher in situational episode 4 compared to 5).

Taken from Rauthmann, J. F. (2020). Person-Environment Relations (Figures). Retrieved from osf.io/ds6mw (CC-BY 4.0 license). 


\section{Figure 2}

A Basic Person-Environment Relations Model (PERM)

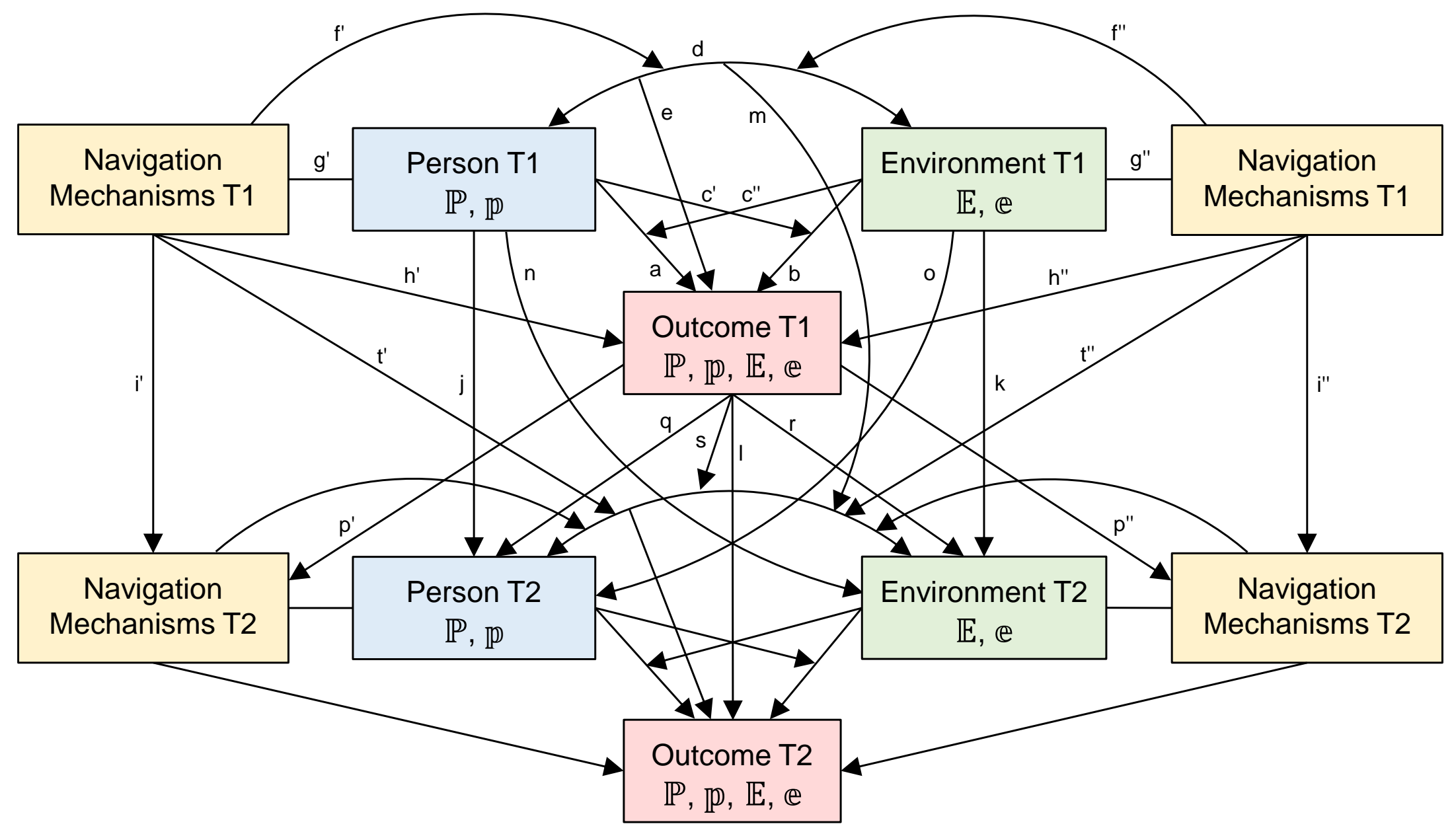

Note. The elements (see Table 2) as well as all effect paths (a-t; see Table 4) are explained in the text.

Taken from Rauthmann, J. F. (2020). Person-Environment Relations (Figures). Retrieved from osf.io/ds6mw (CC-BY 4.0 license). 


\section{Figure 3}

Graphical Examples of Different Forms of Person $\times$ Environment Interactions
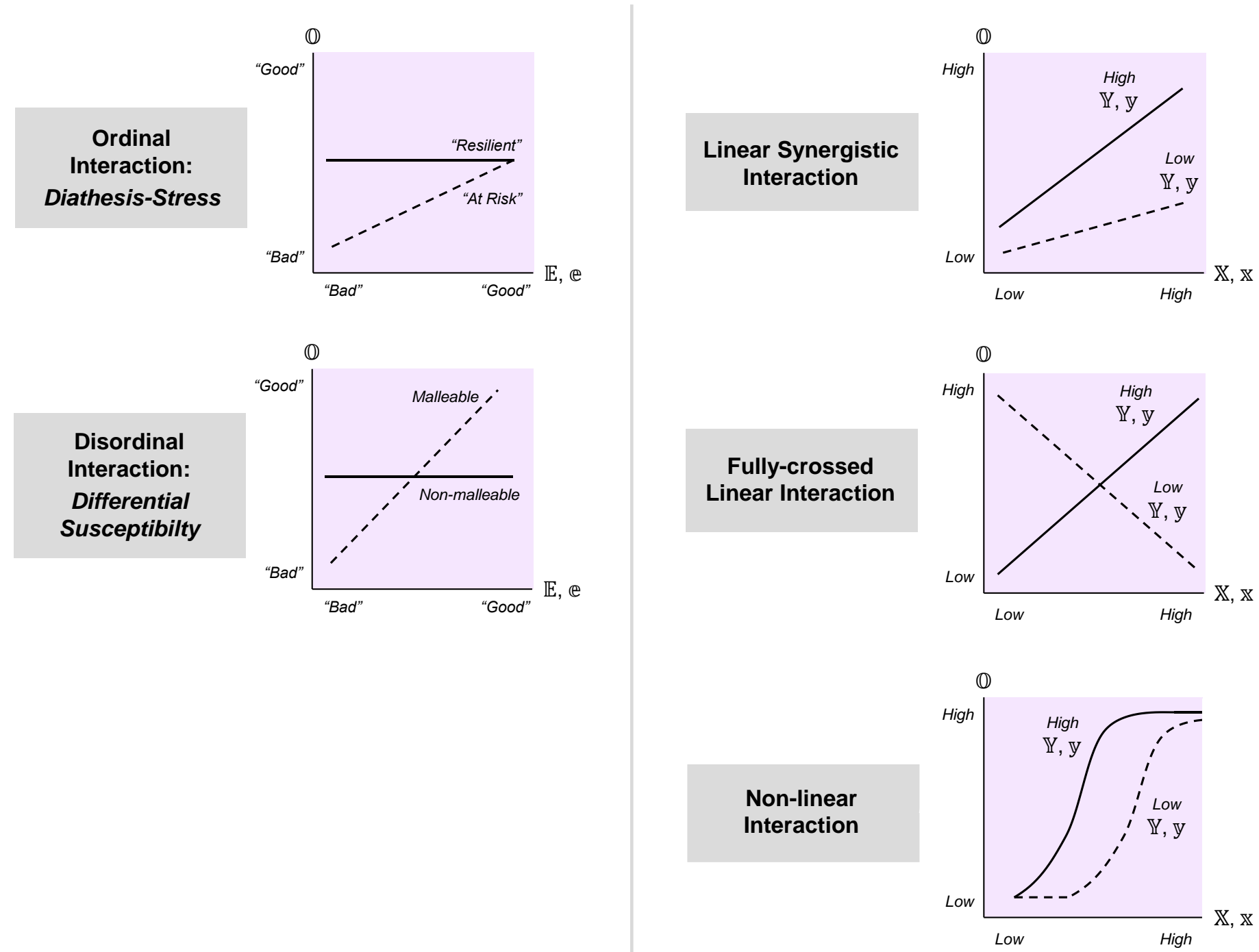

Note. The left two interaction forms are inspired by Figure 1 of Widaman et al. (2012). The right three interaction forms are hypothetical examples, where - depending on whether one wants to analyze one's data from the perspective of persons or environments $-\mathbb{X}$ and $\mathbb{Y}$ could each represent either $\mathbb{P}$ or $\mathbb{E}$, respectively, and $\mathbb{x}$ and $\mathbb{y}$ could represent either $\mathbb{p}$ or $\mathbb{e}$, respectively.

Taken from Rauthmann, J. F. (2020). Person-Environment Relations (Figures). Retrieved from osf.io/ds6mw (CC-BY 4.0 license). 
Figure 4a

Sample Response Surfaces for Linear Person-Environment-Outcome Effect Patterns

$\begin{array}{lllc}\text { Effect } & \text { Regression } & \text { Surface } & \text { Response Surface } \\ \text { Pattern } & \text { Coefficients } & \text { Parameters } & \text { Plot }\end{array}$

\section{Linear Patterns}

Zero

$b_{1}=0$

$b_{2}=0$

$b_{3}=0$

$b_{4}=0$

$b_{5}=0$

$a_{1}=0$

$a_{2}=0$

$a_{3}=0$

$a_{4}=0$

\begin{tabular}{|c|c|c|}
\hline Person-only & $\begin{array}{l}b_{1}=0.2 \\
b_{2}=0 \\
b_{3}=0 \\
b_{4}=0 \\
b_{5}=0\end{array}$ & $\begin{array}{l}a_{1}=0.2 \\
a_{2}=0 \\
a_{3}=0.2 \\
a_{4}=0\end{array}$ \\
\hline
\end{tabular}

Environment-only

$b_{1}=0$

$b_{2}=0.2$

$a_{1}=0.2$

$b_{3}=0$

$b_{4}=0$

$a_{2}=0$

$a_{3}=-0.2$

$b_{5}=0$

$a_{4}=0$

Additive

$$
\begin{aligned}
& b_{1}=0.3 \\
& b_{2}=0.2 \\
& b_{3}=0 \\
& b_{4}=0 \\
& b_{5}=0
\end{aligned}
$$
$a_{1}=0.5$
$a_{2}=0$
$a_{3}=0.1$
$a_{4}=0$
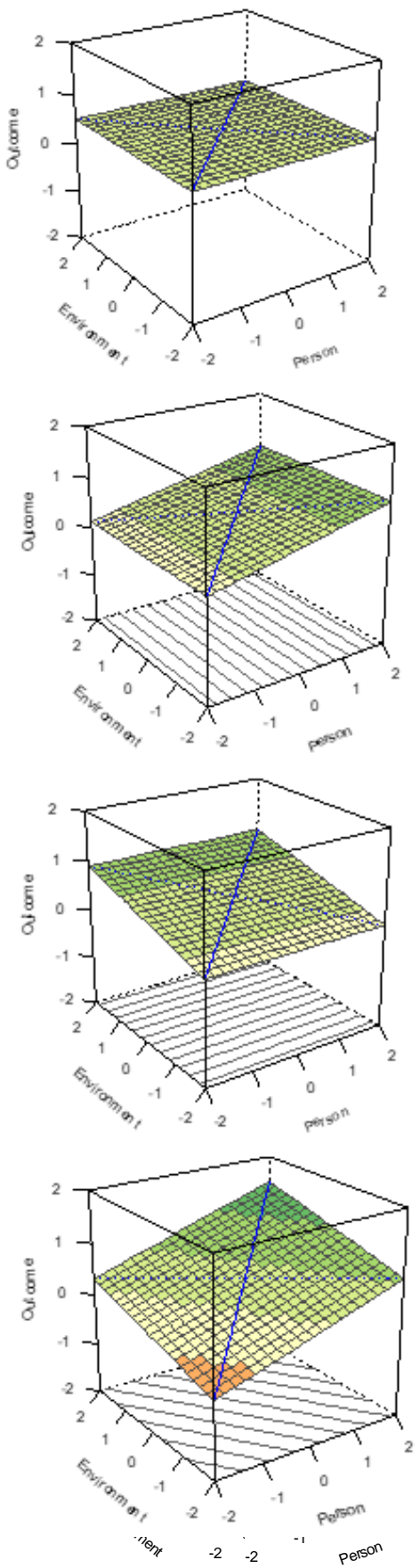
Figure 4b

Sample Response Surfaces for Interactional Person-Environment-Outcome Effect Patterns

\begin{tabular}{lllc} 
Effect & Regression & Surface & Response Surface \\
Pattern & Coefficients & Parameters & Plot \\
\hline
\end{tabular}

Interaction Patterns

Interaction-only

$$
\begin{aligned}
& b_{1}=0 \\
& b_{2}=0 \\
& b_{3}=0 \\
& b_{4}=0.2 \\
& b_{5}=0
\end{aligned}
$$

Person
plus Interaction

$$
\begin{aligned}
& b_{1}=0.2 \\
& b_{2}=0 \\
& b_{3}=0 \\
& b_{4}=0.2 \\
& b_{5}=0
\end{aligned}
$$

\section{Environment plus Interaction}

Additive
plus Interaction

$$
\begin{aligned}
& b_{1}=0 \\
& b_{2}=0.2 \\
& b_{3}=0 \\
& b_{4}=0.2 \\
& b_{5}=0
\end{aligned}
$$

$$
\begin{aligned}
& a_{1}=0 \\
& a_{2}=0.2 \\
& a_{3}=0 \\
& a_{4}=-0.2
\end{aligned}
$$

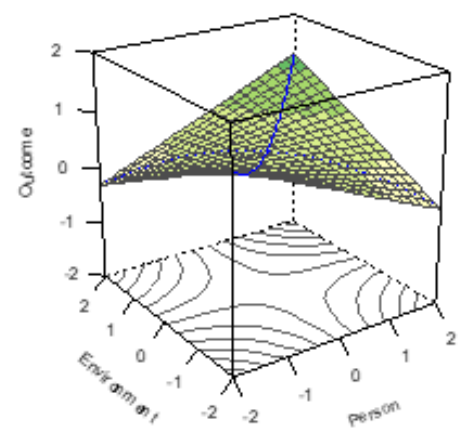

$$
\begin{aligned}
& a_{1}=0.2 \\
& a_{2}=0.2 \\
& a_{3}=0.2 \\
& a_{4}=-0.2
\end{aligned}
$$

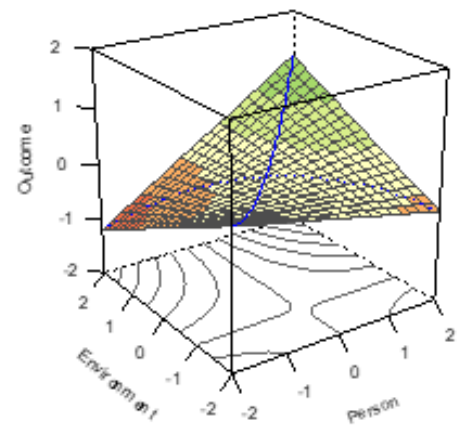

$$
\begin{aligned}
& a_{1}=0.2 \\
& a_{2}=0.2 \\
& a_{3}=-0.2 \\
& a_{4}=-0.2
\end{aligned}
$$

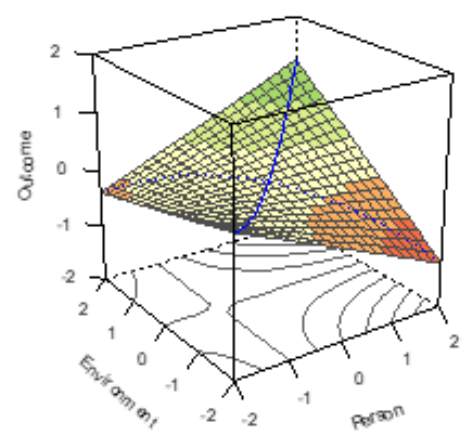

$$
\begin{aligned}
& b_{1}=0.3 \\
& b_{2}=0.2 \\
& b_{3}=0 \\
& b_{4}=0.2 \\
& b_{5}=0
\end{aligned}
$$

$$
\begin{aligned}
& a_{1}=0.5 \\
& a_{2}=0.2 \\
& a_{3}=0.1 \\
& a_{4}=-0.2
\end{aligned}
$$

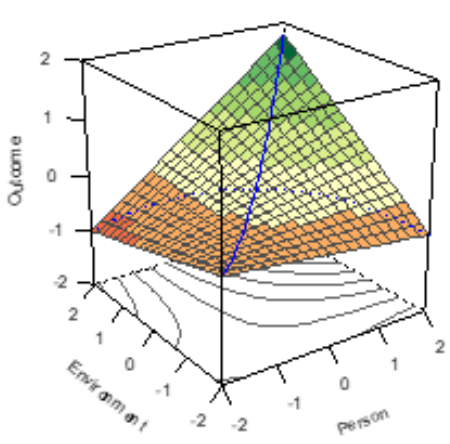


Figure 4c

Sample Response Surfaces for Non-linear Person-Environment-Outcome Effect Patterns

\begin{tabular}{lllc} 
Effect & $\begin{array}{l}\text { Regression } \\
\text { Coefficients }\end{array}$ & $\begin{array}{l}\text { Surface } \\
\text { Parameters }\end{array}$ & $\begin{array}{c}\text { Response Surface } \\
\text { Pattern }\end{array}$ \\
\hline
\end{tabular}

\section{Non-linear Patterns}

\section{Person \\ Strength}

Environment

Strength

Non-linear

Additive

Non-linear Additive

plus Interaction

(Strict Fit Pattern)

$$
\begin{aligned}
& b_{1}=0 \\
& b_{2}=0 \\
& b_{3}=0.2 \\
& b_{4}=0 \\
& b_{5}=0
\end{aligned}
$$

$b_{1}=0$

$b_{2}=0$

$b_{3}=0$

$b_{4}=0$

$b_{5}=0.2$

$b_{1}=0$

$b_{2}=0$

$b_{3}=0.2$

$b_{4}=0$

$b_{5}=0.2$

$b_{1}=0$

$b_{2}=0$

$b_{3}=0.2$

$b_{4}=0.2$

$b_{5}=0.2$ $a_{1}=0$

$a_{2}=0.2$

$a_{3}=0$

$a_{4}=0.2$

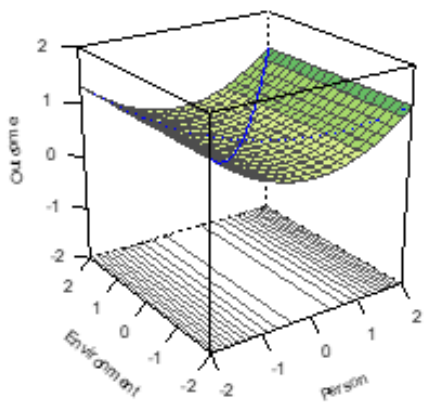

$a_{1}=0$

$a_{2}=0.2$

$a_{3}=0$

$a_{4}=0.2$

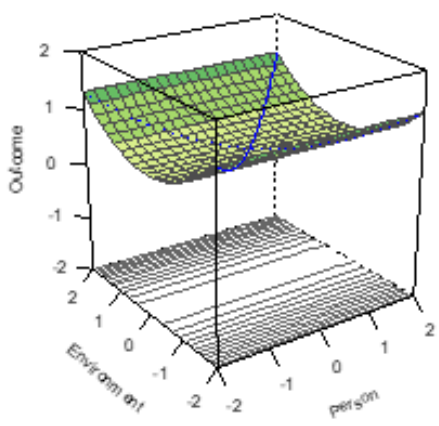

$a_{1}=0$

$a_{2}=0.4$

$a_{3}=0$

$a_{4}=0.4$

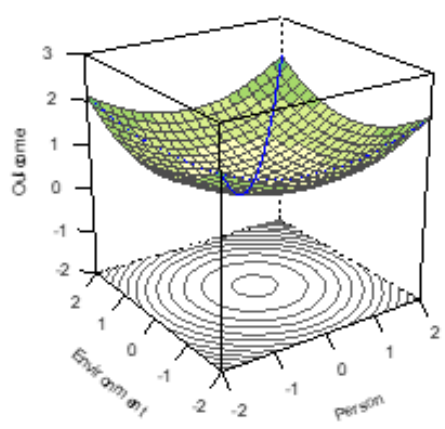

$a_{1}=0$

$a_{2}=0.6$

$a_{3}=0$

$a_{4}=0.2$

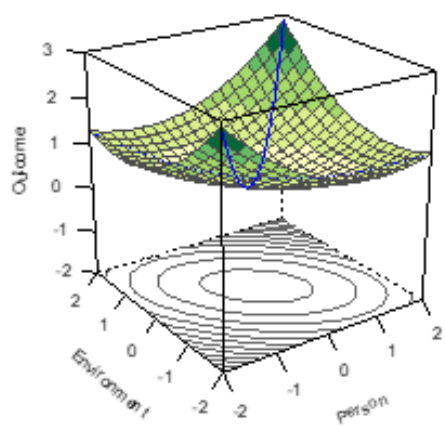


Figure 4d

Sample Response Surfaces for Person-Environment-Outcome Effect Patterns Denoting Fit

\begin{tabular}{lllc}
$\begin{array}{l}\text { Effect } \\
\text { Pattern }\end{array}$ & $\begin{array}{l}\text { Regression } \\
\text { Coefficients }\end{array}$ & $\begin{array}{l}\text { Surface } \\
\text { Parameters }\end{array}$ & $\begin{array}{c}\text { Response Surface } \\
\text { Plot }\end{array}$ \\
\hline
\end{tabular}

Fit Patterns

Strict Fit Pattern

$\begin{array}{ll}b_{1}=0 & a_{1}=0 \\ b_{2}=0 & a_{2}=0 \\ b_{3}=-0.05 & a_{3}=0 \\ b_{4}=0.1 & a_{4}=-0.2 \\ b_{5}=-0.05 & \end{array}$

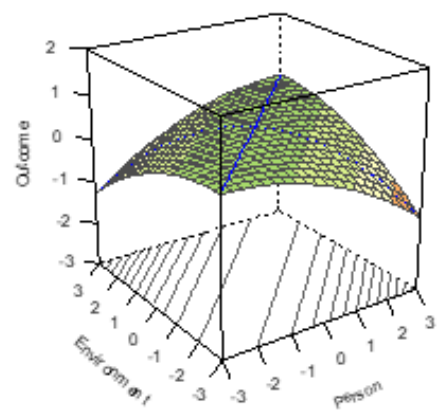

Fit Pattern,

$b_{1}=0.1$

$b_{2}=0.1$

$b_{3}=-0.05$

$a_{1}=0.2$

with linear common

$b_{4}=0.1$

$a_{2}=0$

main effects

$b_{5}=-0.05$

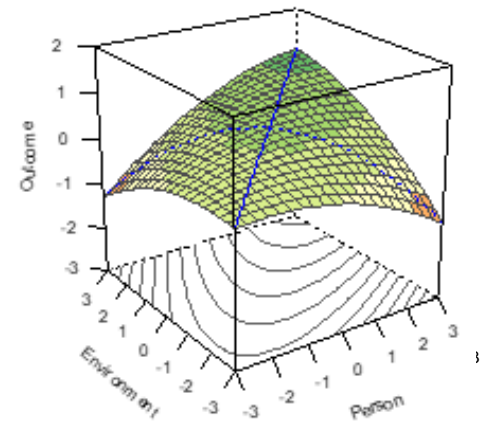

$\begin{array}{lll}\text { Fit Pattern, } & b_{1}=0.1 & a_{1}=0.2 \\ \text { with curvilinear } & b_{2}=0.1 & a_{2}=-0.1 \\ \text { common main effects } & b_{3}=-0.1 & a_{3}=0 \\ & b_{4}=0.1 & a_{4}=-0.3 \\ & b_{5}=-0.1 & \end{array}$

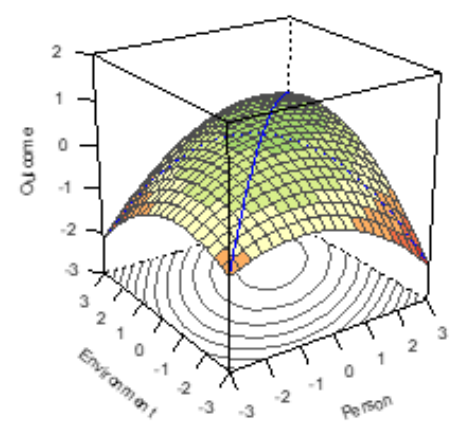

Notes for all Figures $4 a-d$. For all plots, the intercept $\left(b_{0}\right)$ was set to 0.5.

Line of congruence $($ LOC $)=$ solid blue line; line of incongruence $($ LOIC $)=$ dashed blue line.

The three fit pattern examples in Figure 4d are slightly modified from Humberg et al. (2019, Table 3).

All plots were generated by the plotRSA function from the RSA package in R (Schönbrodt, 2016a).

Darker green represents higher and darker orange to red lower values of an outcome in the plots.

$b_{0}=$ intercept $; b_{1}=$ slope of person variable, $b_{2}=$ slope of environment variable, $b_{3}=$ slope of squared person variable, $b_{4}=$ slope of interaction (product term) between the person $\times$ the environment variable, $b_{5}=$ slope of the squared environment variable. $a_{1}=b_{1}+b_{2} ; a_{2}=b_{3}+b_{4}+b_{5} ; a_{3}=b_{1}-b_{2}$; $a_{4}=b_{3}-b_{4}+b_{5}$. See Box 1 for details on polynomial regressions and response surface analysis. Taken from Rauthmann, J. F. (2020). Person-Environment Relations (Figures). Retrieved from osf.io/ds6mw (CC-BY 4.0 license). 


\section{Figure 5}

Hypothetical Examples of Profile-oriented Person-Environment Fit
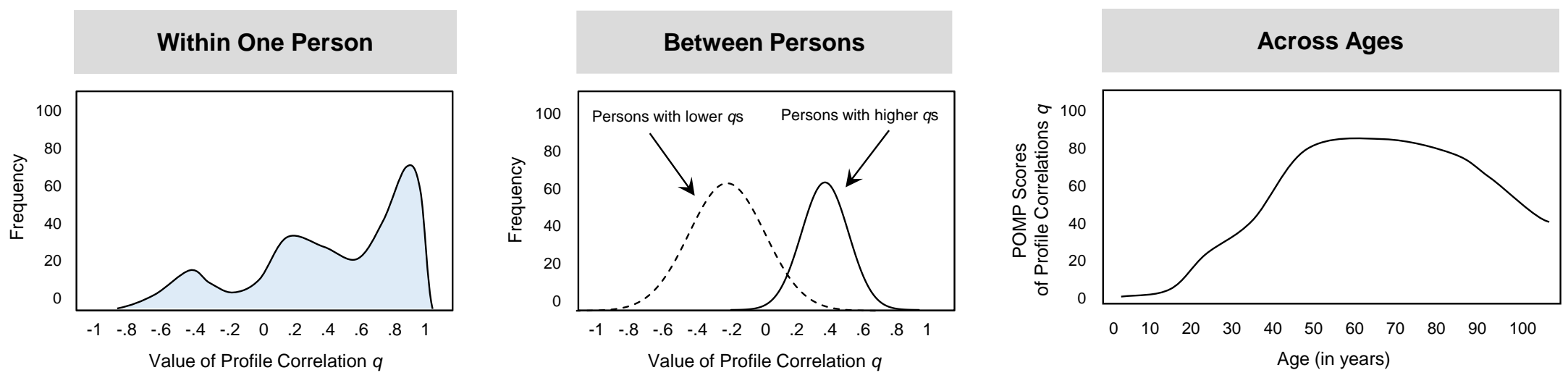

Note. The first panel "Within One Person" depicts an intra-individual distribution of $q$-correlations. Each $q$-correlation is derived by correlating a profile of person variables with a concordant profile of environment variables. Here, many such $q$-correlations could be computed for a person, and hence their frequency distribution with the person can be plotted. In this fictious example, the person rarely has profile correlations below 0 and most above 0 .

The second panel "Between Persons" depicts two density distributions of profile correlations from two different groups of persons (with some overlap, nonetheless). One group (dashed lines) has on average lower $q$-correlations, the other (solid lines) has on average higher $q$ correlations. Each distribution is obtained when examining different individuals' $q$-correlations (which - if measured first repeatedly within each person - may have been mean-aggregated within each person first).

The third panel "Across Ages" depict profile correlations measured for different persons of different ages in cross-sectional data, or from the same persons measured intra-individually across their lifespans in longitudinal data. In both instances, profile correlations on the $y$-axis would have been converted to percent of maximum possible (POMP) scores for better interpretability with other scales from different literatures. The shown age trend or trajectory is hypothetical.

Details can be found in Box 3.

Taken from Rauthmann, J. F. (2020). Person-Environment Relations (Figures). Retrieved from osf.io/ds6mw (CC-BY 4.0 license). 


\section{Figure 6}

A Process Model of Person-Environment Fit and its Consequences

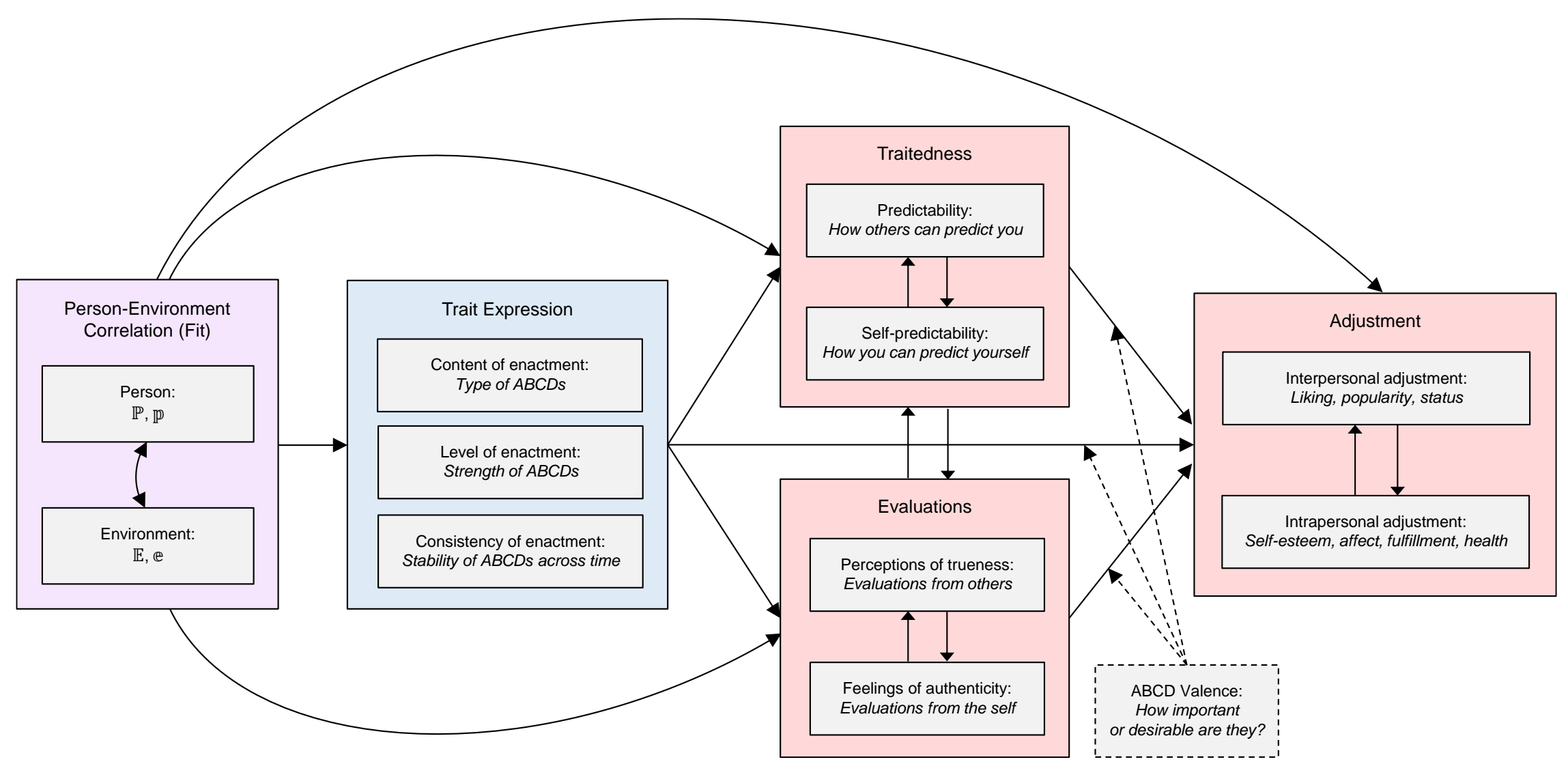

Note. Taken from Rauthmann, J. F. (2020). Person-Environment Relations (Figures). Retrieved from osf.io/ds6mw (CC-BY 4.0 license). 


\section{Figure 7}

Transaction Timescales

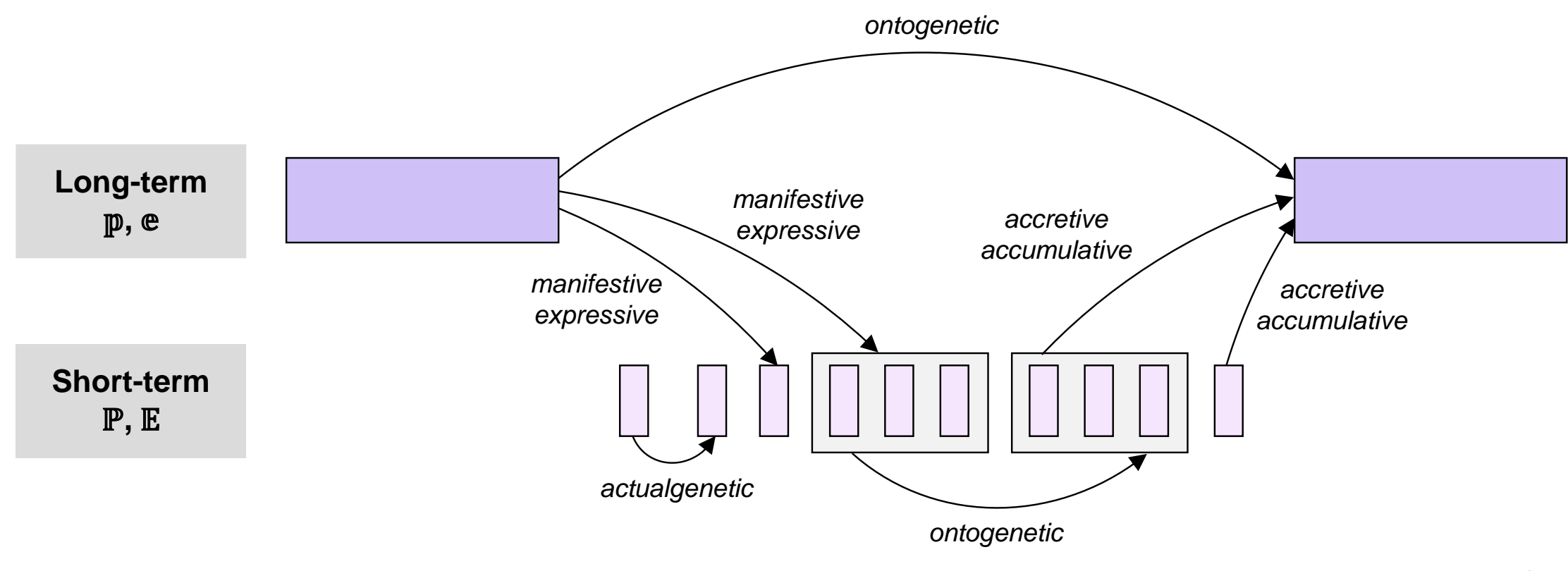

Time

Note. Longer and darker purple boxes (above) reflect more enduring, longer-term variables (person variables: $\mathbb{p}$; environment variables: $巴$ ); shorter and lighter purple boxes (below) reflect less enduring, shorter-term variables (person variables: $\mathbb{P}$; environment variables: $\mathbb{E}$ ); boxes around three shorter-term boxes reflect an aggregate across time (e.g., a mean from a density distribution).

Taken from Rauthmann, J. F. (2020). Person-Environment Relations (Figures). Retrieved from osf.io/ds6mw (CC-BY 4.0 license). 


\section{Figure 8}

Visualization of 16 Possible Person-Environment Transaction Patterns
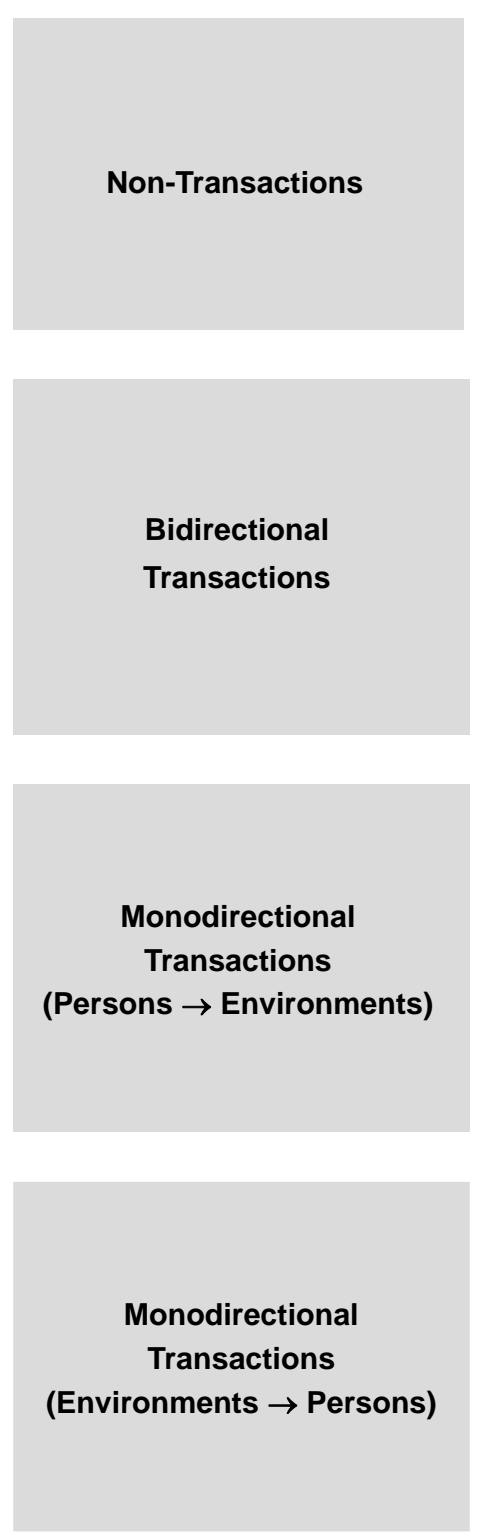
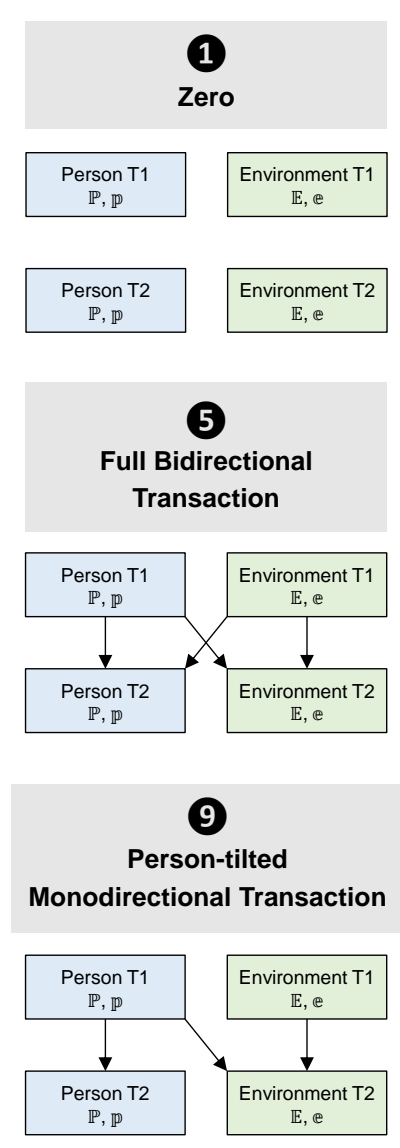

\section{3}

Environment-tilted

Monodirectional Transaction

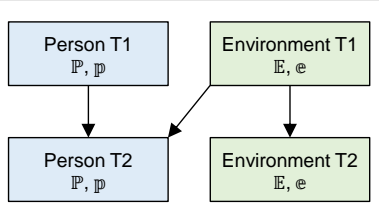

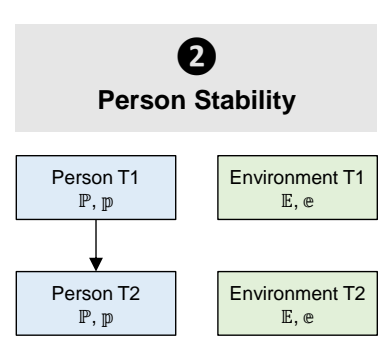
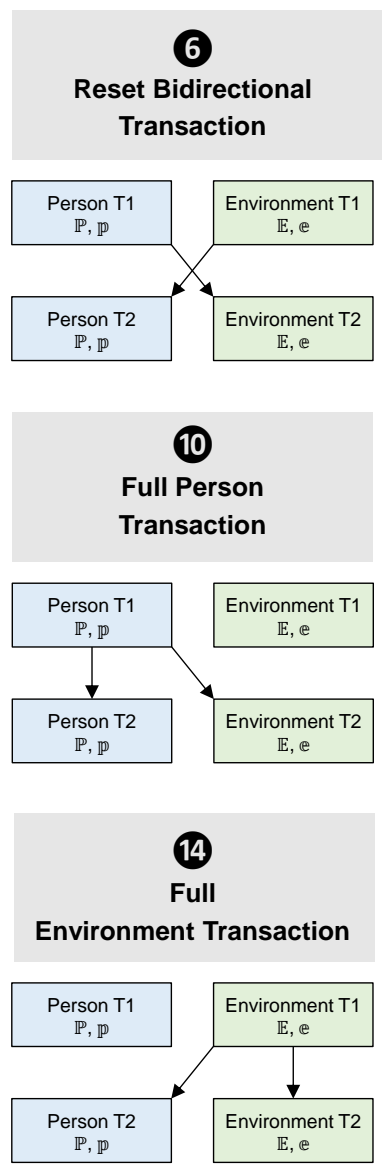

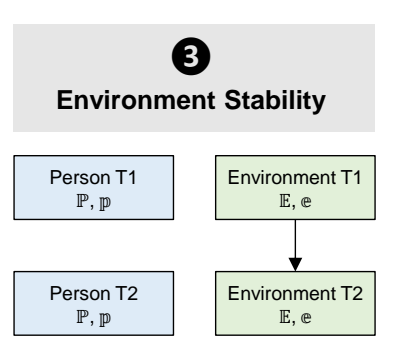

7

Person-tilted Bidirectional Transaction
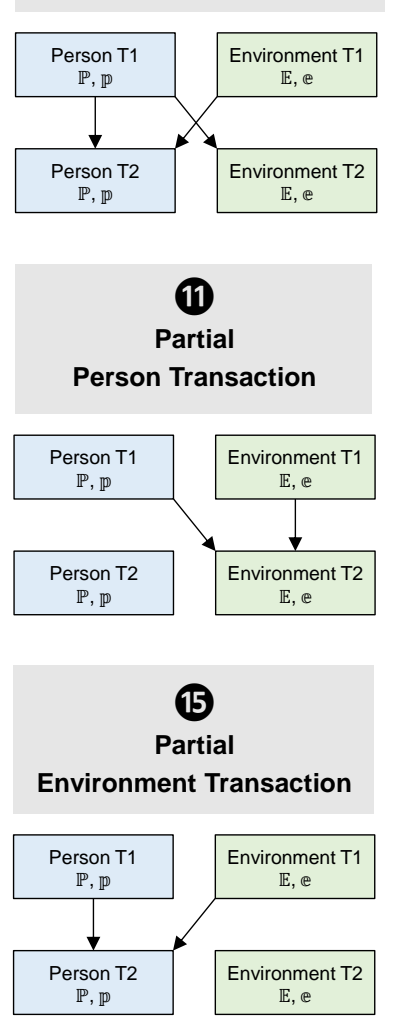
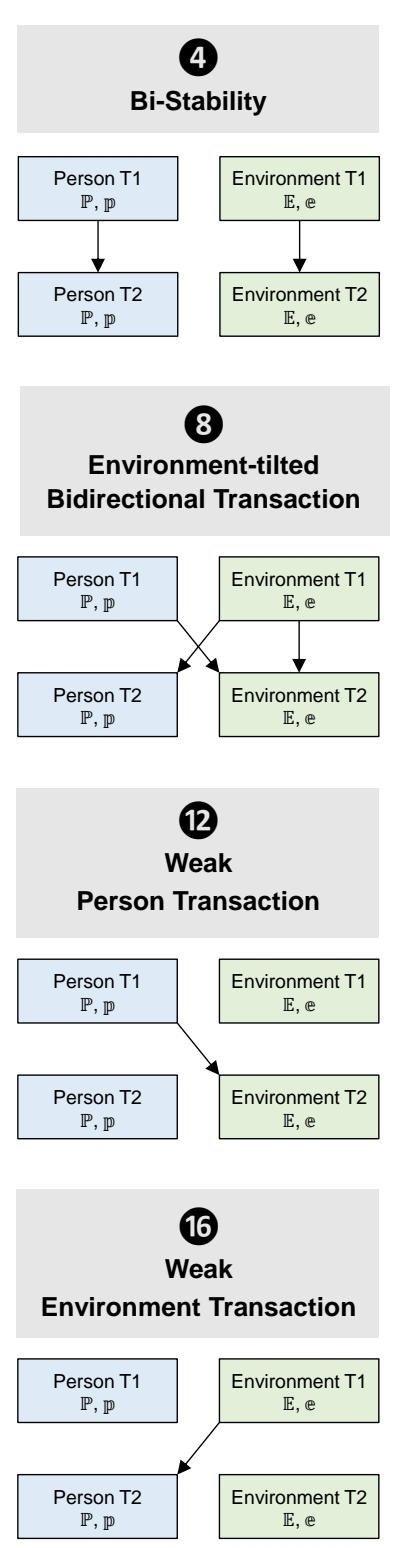

Note. Taken from Rauthmann, J. F. (2020). Person-Environment Relations (Figures). Retrieved from osf.io/ds6mw (CC-BY 4.0 license). 


\section{Figure 9}

A Systematic Dendrogram of Navigation Mechanisms for Person $\rightarrow$ Environment Calibrations

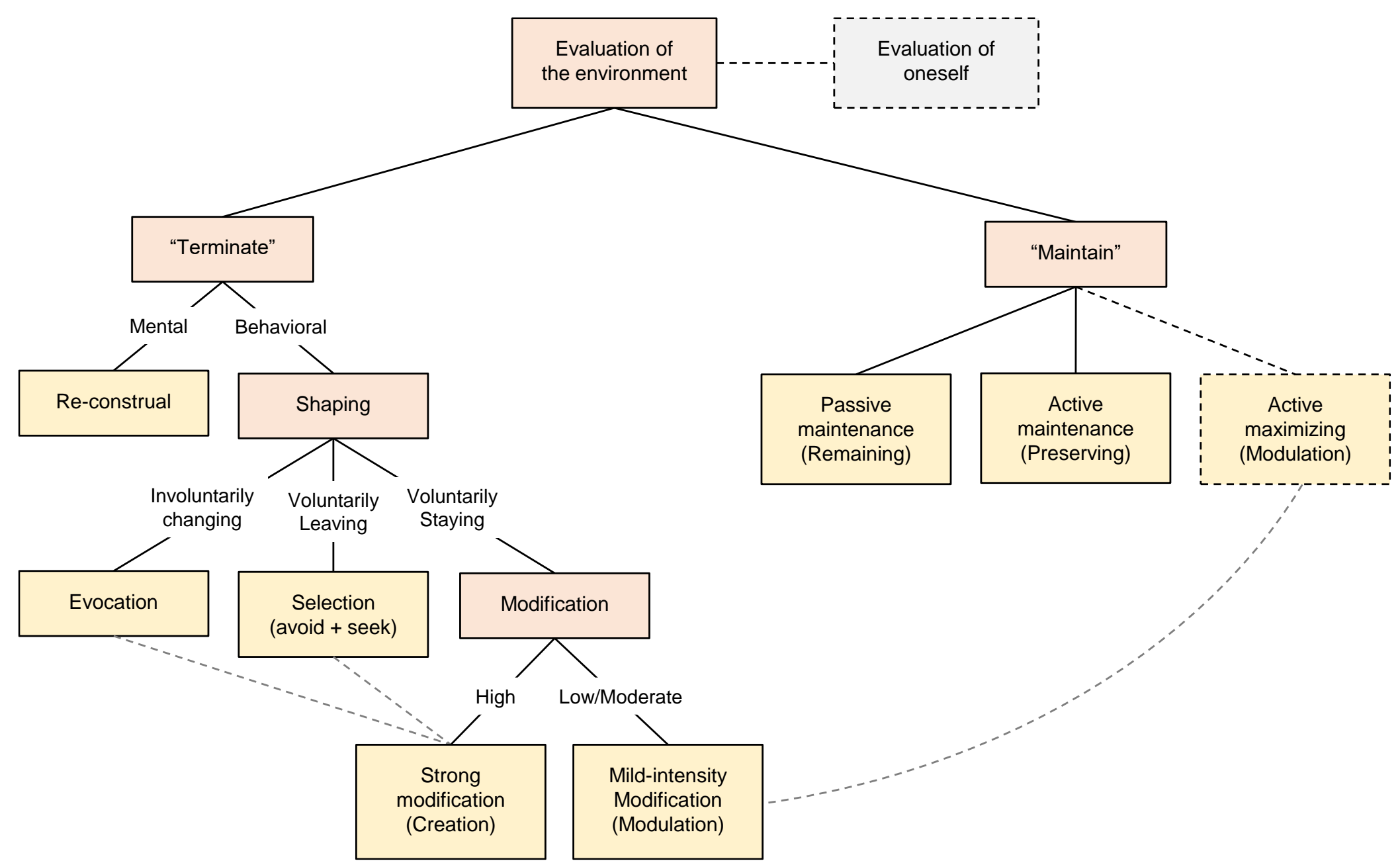

Note. Lighter yellow boxes reflect "end-product" person $\rightarrow$ environment navigation mechanisms that are not decomposed further here. Darker orange boxes reflect navigation mechanisms that can be decomposed further. Dotted light gray lines reflect secondary associations among navigation mechanisms (i.e., they may often co-occur, blend into each other, and/or be difficult to disentangle).

Taken from Rauthmann, J. F. (2020). Person-Environment Relations (Figures). Retrieved from osf.io/ds6mw (CC-BY 4.0 license). 


\section{Figure 10}

Example of Concretizing the PERM to Trace Corresponsive Personality Change Effects

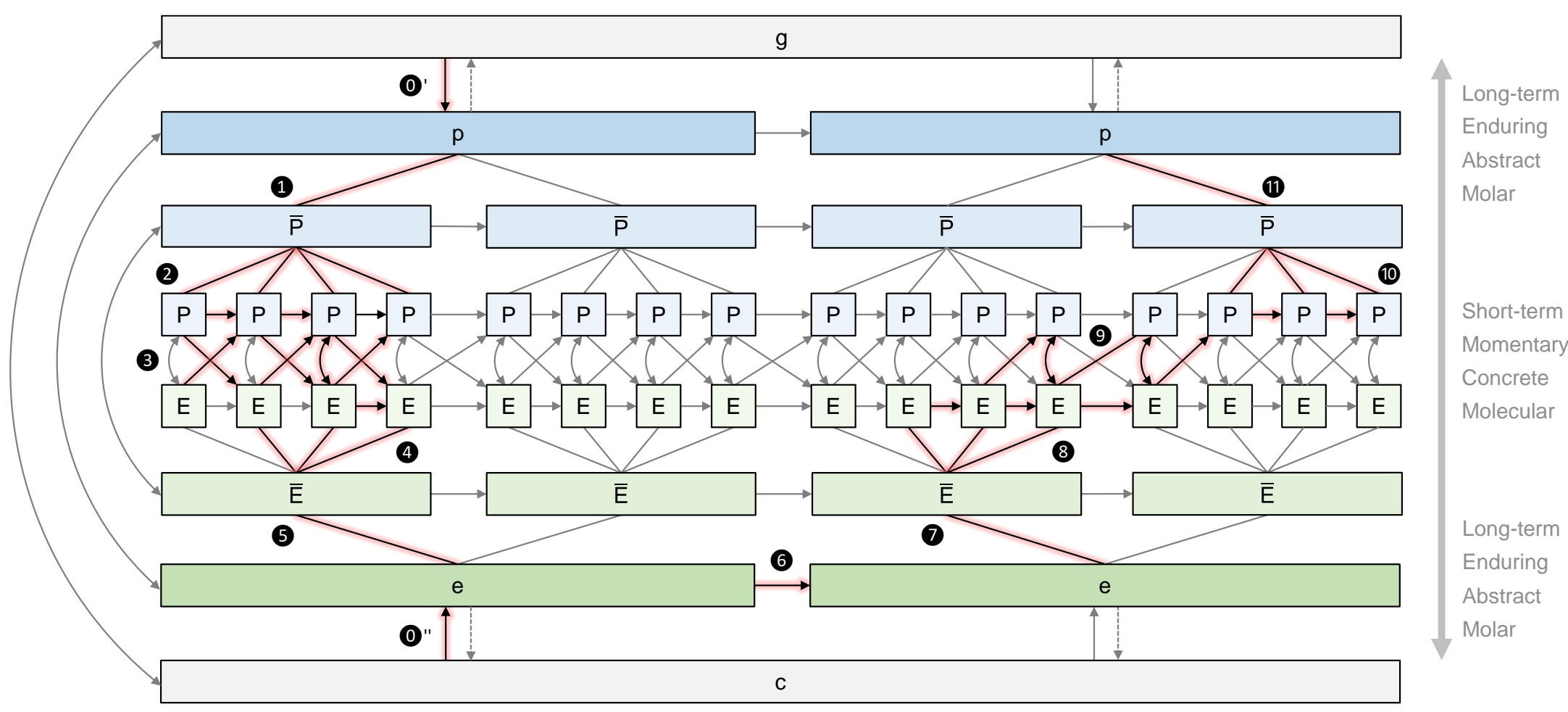

Note. PERM = Person-Environment Relations Model.

Back arrows highlighted in red reflect traceable paths in this example.

Taken from Rauthmann, J. F. (2020). Person-Environment Relations (Figures). Retrieved from osf.io/ds6mw (CC-BY 4.0 license). 